\title{
The predictive value of vital exhaustion in the clinical course after coronary angioplasty
}

Citation for published version (APA):

Kop, W. J. (1994). The predictive value of vital exhaustion in the clinical course after coronary angioplasty. [Doctoral Thesis, Maastricht University]. Datawyse / Universitaire Pers Maastricht. https://doi.org/10.26481/dis.19940127wk

Document status and date:

Published: 01/01/1994

DOI:

10.26481/dis.19940127wk

Document Version:

Publisher's PDF, also known as Version of record

\section{Please check the document version of this publication:}

- A submitted manuscript is the version of the article upon submission and before peer-review. There can be important differences between the submitted version and the official published version of record.

People interested in the research are advised to contact the author for the final version of the publication, or visit the DOI to the publisher's website.

- The final author version and the galley proof are versions of the publication after peer review.

- The final published version features the final layout of the paper including the volume, issue and page numbers.

Link to publication

\footnotetext{
General rights rights.

- You may freely distribute the URL identifying the publication in the public portal. please follow below link for the End User Agreement:

www.umlib.nl/taverne-license

Take down policy

If you believe that this document breaches copyright please contact us at:

repository@maastrichtuniversity.nl

providing details and we will investigate your claim.
}

Copyright and moral rights for the publications made accessible in the public portal are retained by the authors and/or other copyright owners and it is a condition of accessing publications that users recognise and abide by the legal requirements associated with these

- Users may download and print one copy of any publication from the public portal for the purpose of private study or research.

- You may not further distribute the material or use it for any profit-making activity or commercial gain

If the publication is distributed under the terms of Article $25 \mathrm{fa}$ of the Dutch Copyright Act, indicated by the "Taverne" license above, 
THE PREDICTIVE VALUE OF VITAL EXHAUSTION

\author{
IN THE CLINICAL COURSE
}

AFTER CORONARY ANGIOPLASTY 
Cover Design: Jurgen Moorlach

Print: Datawyse/Universitaire Pers Maastricht

CIP-DATA KONINKLIJKE BIBLIOTHEEK, DEN HAAG

Kop, Willem Johan

The predictive value of wital exhaustion in the clinical course after coronary angioplasty / Willem Johan Kop Maastricht: Universitaire Pers Maastricht. - III.

Thesis Maastricht. - With ref. - With summary in Dutch. ISBN 90-5278-126-5

Subject headings: coronary angioplasty / vital exhaustion / coronary diseases. 


\title{
THE PREDICTIVE VALUE OF VITAL EXHAUSTION
}

\author{
IN THE CLINICAL COURSE
}

\section{AFTER CORONARY ANGIOPLASTY}

\section{PROEFSCHRIFT}

ter verkrijging van de graad van doctor

aan de Rijksuniversiteit Limburg te Maastricht,

op gezag van de Rector Magnificus, Prof. Dr. H. Philipsen,

volgens het besluit wan het College van Dekanen,

in het openbaar te verdedigen op

donderdag 27 januari 1994 om 16.00 uur

door

\section{Willem Johan Kop}

geboren op 1.1 augustus 1963 te Zoetermeer

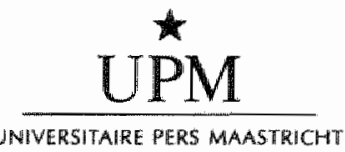




\section{Promotor:}

Prof. Dr. A.P.W.M. Appels

\section{Co-promotor:}

Dr. F.W. Bär

\section{Beoordelingscommissie:}

Prof. Dr. J. Jolles (voorzitter)

Dr. A. Gorgels

Prof. Dr. M.A. van den Hout

Prof. Dr. R.S. Reneman

Prof. Dr. J. Siegrist (Heinrich Heine Universität, Düsseldorf, Duitsland)

Financial support by the Netherlands Heart Foundation and the Dutch Organization for Scientific Research for the publication of this thesis is gratefully acknowledged. 
You're walking. And you don't always realize it, but you're always falling.

With each step, you fall forward slightly. And then catch yourself from falling.

over and over, you're falling.

And then catching yourself from falling.

And this is how, you can be walking and falling at the same time.

Laurie Anderson,

part of: "Walking \& Falling".

from: 'Big Science', 1982. 



\section{Contents.}

1.

1.1.

1.1.1.

1.1.2.

1.1.3.

1.2

1.2.1.

1.2.2.

1.3.

1.3 .1 .

1.3.2.

1.3.3.

1.4.

1.4 .1 .

1.4.2.

1.4 .3 .

1.5.

2.

2.1 .

2.1.1.

2.1.2.

2.1.3.

2.1.4.

2.2 .

2.2.1.

3.

3.1.

3.1.1.

3.1.2.

3.1 .3$.

3.2.

3.3.
Introduction

The origins of exhaustion before myocardial infarction $\ldots \ldots \ldots \ldots \ldots$

Cardiological views

Psychiatric views

Psychological views.

Vital exhaustion as a precursor of myocardial infarction.

Prospective studies.

Case-control studies

Psychosocial precursors of wital exhaustion

Type A behavior and Hostility

Life events

Owerwork and lack of sleep

Vital exhaustion and other tiredness-related constructs

Depression

Burnout:

The Chronic Fatigue Syndrome

Research questions

Methods and Rational

Vital exhaustion in cardiological patients 31

Hypothesis 1

Hypothes is 2

Hypothes is 3

Hypothes is 4

Blood clotting factors and vital exhaustion

Hypothesis 5

The relationship between the severity of coronary artery disease and feelings of exhaustion

Methods. 48

Patients

Vital exhaustion

Coronary artery disease and left ventricular function

Results 50

Discussion 53 
4.1. Methods .............................. 56

4.1.1. Patients

4.1.2. Procedure

4.1.3. Severity of coronary artery disease and clinical control variables

4.1.4. Siatistical analysis

4.2. Results . . . . . . . . . . . . . . . . . 58

4.2.1. Exhaustion before PTCA

4.2.2. The effect of PTCA on exhaustion

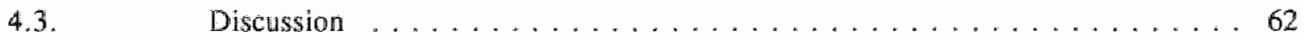

5. Vital exhaustion predicts new cardiac events after successful coronary angioplasty $\ldots \ldots \ldots \ldots \ldots \ldots \ldots 6$

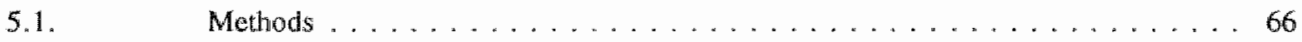

5.1.1. Patients

5.1.2. Patient characteristics

5.1.3. Follow-up

5.1.4. Statistical analysis

5.2. $\quad$ Results ............................. 70

5.2.1. Clinical predictors of new cardiac events

5.2.2. The predictive value of vital exhaustion

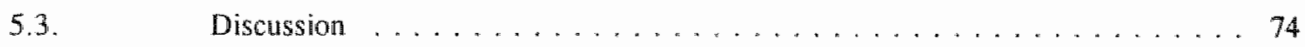

6. The consequences of vital exhaustion for blood coagulation and fibrinolysis $\ldots \ldots \ldots \ldots \ldots \ldots \ldots \ldots 77$

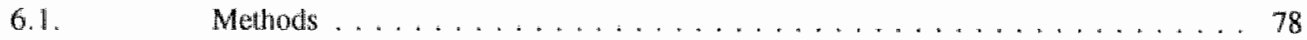

$\begin{array}{ll}6.1 .1 . & \text { Selection of subjects } \\ 6.1 .2 . & \text { Blood collection }\end{array}$

6.1.3. Measurements

6.1.4. Statistical analysis

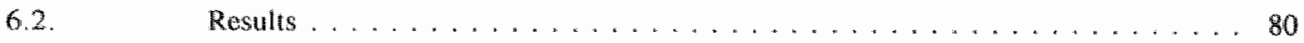

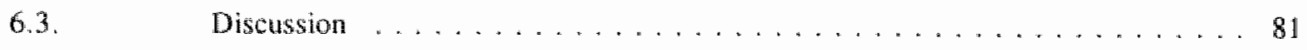


7. Psychobiological mechanisms of the relationship between vital exhaustion and acute coronary syndromes . . . . . . . . . . . . . . . . . . . 85

7.1. The pathogenesis of coronary lesions $\ldots \ldots \ldots \ldots \ldots \ldots$

7.2. Mechanisms of restenosis after coronary angioplasty $\ldots \ldots \ldots \ldots 7$

7.3. Factors that contribute to development of acute coronary syndromes . . . . 89

7.4. The relationship between vital exhaustion and the biological . . . . . . 91 mechanisms of acute coronary syndromes

7.5. The relationship between vital exhaustion and immunological aspects . . . . 94 of atherosclerosis

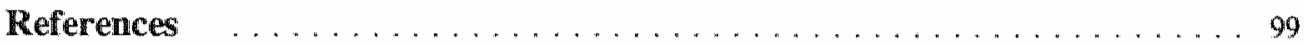

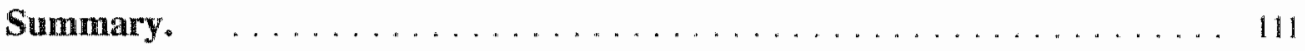

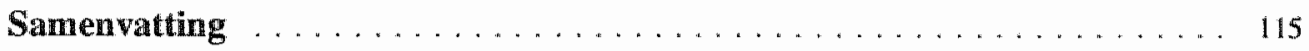

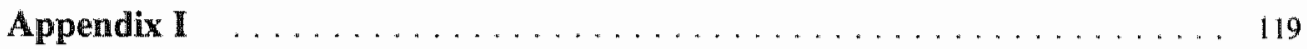

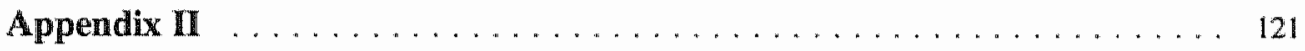

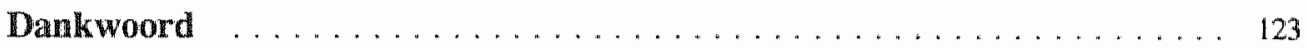

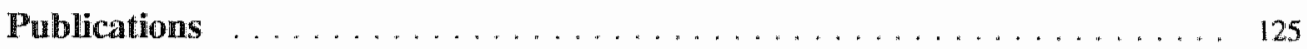

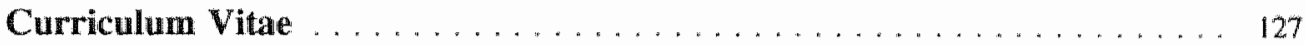





\section{Chapter 1.}

\section{Introduction.'}

The studies reported in the chapters to come concern the relationship between feelings of exhaustion and the progression of coronary artery disease. The present chapter begins with an overview of the premonitory symptoms of myocardial infarction and sudden cardiac death. The excessive tiredness and feelings of general malaise which often precede myocardial infarction are discussed from three perspectives: cardiology, psychiatry, and psychology.

Then, the development of the instrument that assesses this prodromal state is presented: the Maastricht Questionnaire. It is argued why the label 'vital exhaustion' is chosen to describe the extreme tiredness before myocardial infarction, making use of the data of prospective and case-control studies. In addition, these studies are used. to estimate the magnitude of the association between vital exhaustion and future cardiac events.

The third section presents a theoretical frame-work to explain the genesis of vital. exhaustion. It is suggested that vital exhaustion is the end-stage of prolonged psychological tension.

In the fourth section, the similarities and distinctions are discussed between vital exhaustion and other psychological states which contain feelings of exhaustion as a key feature (i.e., depression, burnout, and the chronic fatigue syndrome).

The last section of this chapter concludes with the research questions of the present investigation.

\subsection{The origins of exhaustion before myocardial infarction.}

Excess fatigue and feelings of general malaise are among the most prevalent precursors of myocardial infarction and sudden cardiac death. Estimates of the percentage of people who experienced "undue fatigue" or "lack of energy" before a cardiac event range from 13\% to 70\% (Wolf, 1969; Kuller et al., 1972; Simon et al., 1972; Alonzo et al., 1975; Feinleib et al., 1975; Gillum et al, 1976; Fraser, 1978; Rissanen et al., 1978; Stowers \& Short, 1970; Thiele et al., 1985; Klaeboe et al., 1987). One of the reasons for this wide range is the absence of consensus as to what exactly should be asked to coronary patients in order to examine this issue. Two case reports are

A modified version of this chapter is in press in: Maes $\mathbf{S}$ ed. International Review of Health Psychology. London: Wiley. 
presented in Appendix I to illustrate the behaviors and feelings that are characteristic of patients prior to myocardial infarction. There are several conceptual ways to address the origins of the tiredness before acute coronary events, these are roughly classified as cardiological, psychiatric, and psychological views.

\subsubsection{Cardiological views.}

Fatigue is an aspecific symptom, which is present in numerous clinical conditions. Therefore, the complaint of fatigue can be of diagnostic importance in cardiology only if the details of this symptom are known. In patients suffering from manifest heart disease feelings of fatigue may reflect a failure of the left ventricle to increase the stroke volume when there is need for a higher cardiac output. This failure may be due to an impaired oxygen supply to the myocardium or to an impaired contractility of the left ventricle. "As a rule, the fatigue due to heart disease is related to effort, whereas the fatigue of anxiety or depression is constantly present" (Hurst et al., 1990). The textbook of Brandenburg makes a similar distinction: "When fatigue is chronic, is present on awakening and persists all day without change, the cause is most likely not cardiac. In such cases, anxiety or increased emotional tension and poor sleep are the main causes; anaemia and other chronic disease states such as hypothyroidism should be considered. Conversely, if the symptom is new or of recent onset and tends to develop during the day to the point of physical exhaustion without any effort, a cardiac failure-related low output with subnormal increment with exercise is suggested" (Brandenburg et al., 1987).

Few cardiological studies have directly addressed the question of the origin of the excessive tiredness prior to myocardial infarction or sudden death. Kuller (1978) has related the prodromal symptomatology of sudden death to pathological changes observed at postmortem examination. There was no relationship between any of the prodromal symptoms and the presence of acute pathological changes as manifested by coronary thrombosis. With regard to the feelings of fatigue Kuller notes: "the basis of the fatigue syndrome has not been determined. No information is currently available describing the relationships of the fatigue syndrome to any specific pathophysiological changes. The most interesting hypothesis related to the fatigue syndrome suggests that fatigue is related to a decrease of left ventricular function, possibly secondary to increased myocardial damage. An equally interesting and alternative hypothesis is that the fatigue represents a manifestation of depression" (Kuller, 1978). Freeman and Nixon observed an association between symptoms of exhaustion (waking up unrefreshed from sleep and poor energy levels) and the number of symptomatic episodes of myocardial ischaemia during ambulatory monitoring. This may point to a reduced myocardial oxygen supply in exhausted individuals.

In summary, if fatigue in patients with coronary artery disease has a cardiac origin, this is believed to be caused by either impaired myocardial oxygen supply due to coronary atherosclerosis or a reduced pump-function of the heart. In addition, tired- 
ness may also be a side effect of medication, especially in the case of diuretics and beta-blocking agents.

\subsubsection{Psychiatric views.}

There are several psychiatric descriptions of the mental precursors of myocardial infarction. The assessments of patients' experiences reveal common features, but there are differences in the terminology used. The labels that have been used include: "hidden withdrawal and masked depression" (Fischer et al., 1964), "a combination of depression and arousal effects" (Greene et al., 1962), and "pseudo neurasthenic syndrome" (Polzien \& Walter, 1971). This mental state reflects a breakdown of the defence mechanisms, especially of compulsive striving for achievement and mastery (Arlow, 1945).

Hahn performed the first quantitative psychiatric study on the short-term precursors of myocardial infarction. It was observed that coronary patients were significantly more compulsive, involuntary counters of trivial things such as houses or trees than controls were. Hahn interpreted this compulsive behavior as a defence mechanism directed against a depressive ambivalence conflict. Upon decompensation of this defence through conflicts or stress, the underlying anxieties and depressive ways of coping with conflicts become manifest. By means of a questionnaire Hahn looked into the ways in which this decompensation was experienced by coronary patients. The vast majority reported to have experienced increasing irritability, strong mood changes, increased sweating and the general feeling of not being well (Hahn, 1971).

The first prospective psychiatric study on the mental precursors of myocardial infarction was published by Crisp, Queenan and D'Souza (1984). The database of this study comprised patients aged 40-65 years who were registered at a general practice in London. Nearly all participants completed the Crown - Crisp Experiential Index. During the five year follow-up period, 26 men were admitted to the hospital with a confirmed diagnosis of myocardial infarction. Scores on the inventory were compared with those of the remainder of the study population. Differences between groups in scores on the individual items were examined and a discriminant analysis was carried out in order to devise a linear combination of variables that discriminated the groups most accurately. Twelve items were found to discriminate between individuals destined and those not destined for myocardial infarction. The authors interpreted this item subset as reflecting "a state of sadness, coupled with loss of libido and exhaustion" (Crisp et al., 1984). Although the study has the advantages stemming from a longitudinal design the findings should be interpreted with caution because chance fluctuations may strongly influence the results of a discriminant analysis. In a new analysis of the same database, Haines et al. (1987) observed that the scales "obsessional neurosis", "depression" and "hysteria" were predictive of new ischemic heart disease. However, these scales lost their predictive power when they were controlled for phobic anxiety. 
In general, the psychiatric conception of the tiredness prior to myocardial infarction assumes that this state reflects a decompensation of defenses which results in subsequent anxious and depressive reactions to psychological stressors.

\subsubsection{Psychological views.}

During the last two decades health psychology has spent much attention to the Type A Behavior Pattern as related to coronary artery disease. This is an owert behavior pattern composed of feelings of time pressure, competition, impatience, aggression, and easily provoked hostility. The Western Collaborative Group Study revealed that healthy middle-aged men who were Type $A$ at screening were twice as likely to suffer a myocardial infarction in the subsequent eight and a half years than men without this behavior pattern (Roseruman et al., 1975).

On the basis of the Type A theory, Glass developed a psychological model in which Type A individuals were supposed to pass a state of frustration and exhaustion, a "prodromal depression", preceding myocardial infarction. The basic assertion was that "Type A individuals exert greater efforts than their Type B counterparts to control stressful events that are perceived as threats to their sense of control. These active coping attempts eventually extinguish, for without reward, the relentless striving of the Type $\mathrm{A}$ individual leads to frustration and psychic exhaustion, which culminates in a reduction of efforts at control" (Glass, 1977). Experimental studies were in line with this model (Krantz et al, 1974). Arguments in support of the interaction between Type A behavior and helplessness as a prodromal phase of clinically manifest coronary artery disease will be presented in the studies described below (section 1.3.).

A somewhat different model has been suggested by Bruhn who conceives myocardial infarction as the endpoint of a history of "emotional drain". "Emotional drain is defined as a frustrating, long term involvement of the individual's mental processes in his attempt to live with or cope with some life fact or conflict which involved some deeply ingrained aspect of the individual, such as his values, beliefs, self-concept, or interpersonal relationships... Emotional drain implies a nearly constant state of mental preparedness on the part of the individual to cope with this conflict... to the point where the total organism is left in a state of physical and mental exhaustion... Thus, the life long conflicts that are perceived as to be unsolvable to an individual whose mental resources are continuouslly mobilized as if he were attempting to solve them may eventually leave him in a state of mental and physical exhaustion. It is this stage that may set the stage for myocardial infarction and sudden death" (Bruhn et al,, 1968).

The psychological model of the development of exhaustion is elaborated upon further in section 1.3. For the moment, it suffices to summarize that the psychological perspective of exhaustion prior to myocardial infarction attributes its origin to a breakdown in adaptation to prolonged psychological stress. 


\subsection{Vital exhaustion as a precursor of myocardial infarction.}

The initial conceptualization of vital exhaustion was formed by Appels and colleagues at the department of Medical Psychology in Rotterdam, the Netherlands during the second half of the 1970s. At that time it was felt that it would not be elegant to adhere to the usual parsimonious strategy of selecting an established psychological test and, then, to examine its predictive power. This would bring about the disadvantage of a premiature item-selection, the more since the phenomenology of the mental state before myocardial infarction was not well documented. Therefore, a large number of interviews with coronary patients were carried out. The patients' responses were used to construct an initial scale, which was subsequently tested in two case-control studies (Appels et al., 1980; Verhagen et al., 1980).

Thirty-seven items were found to discriminate between coronary cases and a healthy control group. This scale has been called "form A of the Maastricht Questionnaire" $(\mathrm{MQ}-\mathrm{A})^{2}$. At that stage of the "data driven" approach it could not yet be decided if these symptoms reflected (sub)clinical heart disease, a psychiatric syndrome, or a projection of current feelings onto the past.

The first attempt to obtain more insight into the construct assessed by this scale was made in a study on approximately 50 bus-drivers. Those who had elevated scores on the MQ-A were interviewed by an experienced clinical psychologist (Dr. M. Ceha). This group was described as "vitally exhausted". The label fitted well with the experiences of the research group of Appels and hence the construct assessed by the MQ-A was labelled as vital exhaustion. This had the additional advantage of avoiding a premature psychiatric label. Vital exhaustion has three defining characteristics: 1) feelings of excess fatigue and lack of energy; 2) increased irritability; and 3) feelings of demoralization (Appels, 1992).

Subsequently, a series of prospective and case-control studies were performed to examine the magnitude of the relationship between vital exhaustion and myocardial infarction. These studies will be described below. In addition, the relationship between vital exhaustion and cardiac events in women will be illustrated as well as the strength of the association in other countries than the Netherlands. The problem of the use of questionnaires will also be discussed and some initial data on the recently developed Interview Vital Exhaustion are presented.

\subsubsection{Prospective studies.}

The municipal health authority of the city of Rotterdam started a health check-up of the city employees in 1979. Participants were 3877 male employees aged $39-65$

Although the basic ideas about vital exhaustion was formulated at the time when Appels worked in Rotterdam, he moved to Maastricht at about the same time as the prospective study started. This has lead to Maastricht instead of Rotterdam as the prefix to the Questionnaire. 
years, who were screened between january 1979 and december 1980 . The medical examination included measurements of blood-pressure, cholesterol, relative weight, smoking, and an assessment of chest pain using the Rose Questionnaire (Rose et al., 1982). In order to exclude subjects with previous myocardial infarction, a resting electrocardiogram was added to the medical examination. All subjects completed the MQ-A. In addition to the 37 items, 21 new items derived from a new series of clinical interviews were added to this scale. The cohort was followed up for an average period of 4.2 years and the occurrence of myocardial infarction during follow-up was registered.

Twenty-one subjects died of myocardial infarction, and a well documented non-fatal myocardial infarction occurred in 38 subjects. Subjects were assigned the label 'exhausted' if the MQ-A score was above the median. The relative risk of exhaustion for a fatal or non-fatal myocardial infarction was $2.28(\mathrm{p}<0.01)$; in this analysis Appels et al. (1987, 1988, 1989) controlled for age, cholesterol, smoking, and blood pressure. An analysis of the observed association between vital exhaustion and future myocardial infarction against the time interval showed a sharp decrease in the relative risk for events occurring in the first, second, third, or fourth year of follow-up. Among those who scored below the median no myocardial infarctions occurred in the first year of follow-up. To allow statistical analysis, the value of 1.00 was added to each cell, which resulted in an age-adjusted estimated relative risk of 10.05 in the first year of follow-up, while for the following three years the estimates were $2.23,3.04$, and 0.68 , respectively. These data suggest that exhaustion is a short-term risk indicator for myocardial infarction. No relationship was observed between elevated exhaustion scores at screening and future cancer or gastrointestinal disease. This suggests that vital exhaustion is specifically related to near future cardiac events.

An item analysis was performed to construct a definite questionnaire for the assessment of vital exhaustion based on predictive validity. It appeared that the predictive power of the MQ-A was due to 16 of the 37 items. Furthermore, 8 of the 21 added items were predictive, resulting in a total of 24 items that were significantly related to future myocardial infarction. Because there were doubts as to the content validity of three items, these were removed from the final scale. Based upon this item analysis the final form of the MQ (form B) consists of 21 items and is displayed in Appendix III (Appels et al., 1987).

Given the paucity of prospective data on exhaustion as a precursor of cardiac disease it was investigated whether a similar observation could be made using the database of a different prospective study (Appels \& Otten, 1992). In the KaunasRotterdam Intervention Study 3365 males participated in a cardiovascular screening program in Rotterdam between 1972 and 1974. This cohort was followed up during an average period of 9.5 years. All participants completed the Reeder Stress Scale at screening. Although presently this scale is not the preferred instrument to assess psychological stress, it has one item which is close to the concept of vital exhaustion: "I am completely exhausted, both mentally and physically at the end of the day". 
Among subjects without manifest coronary artery disease or a history of myocardial infarction at screening, 69 subjects died as a consequence of myocardial infarction. The cumulative incidence of cardiac death was found to be $45 / 1000$ in the exhausted group, $30 / 1000$ in the intermediate group, and $26 / 1000$ in the group that was not exhausted. Those who felt exhausted at screening were found to be at elevated risk most markedly at the beginning of the follow-up period. The hazard ratios for exhaustion were $8.96,6.33,4.47$ and 3.16 for the first $10,20,30$ and 40 months of followup, respectively. Thereafter, the association lost its statistical significance. Cox regression analysis showed a highly significant interaction between time until event and exhaustion upon the risk of cardiac death. These findings support the hypothesis that feelings of exhaustion increase the risk of fatal myocardial infarction in men who are free of manifest coronary artery disease.

The results of these prospective studies are corroborated in a study by Siegrist, who followed 416 blue collar workers, aged 25 - 55 years, during 6.5 years. It was found that a state of exhaustive coping reflecting frustrated but continued efforts and associated negative feelings such as disproportionate irritability and inability to withdraw from work obligations substantially increased the risk of myocardial infarction when controlled for all standard risk factors (Siegrist et al., 1990).

The previous studies show that feelings of exhaustion precede manifest heart disease in apparently healthy males. However, since the prospective studies used the resting electrocardiogram in an attempt to exclude patients with coronary disease, it can no: be ruled out that subclinical coronary artery disease explains the association between vital exhaustion and future cardiac events. To exclude coronary artery disease, a detailed history of the symptoms should have been obtained in addition to a physical examination and an exercise electrocardiogram. Therefore, exhaustion in future myocardial infarction subjects may have been caused by impaired coronary perfusion at screening, and it is this perfusion deficiency that could explain both the presence of vital exhaustion prior to myocardial infarction as well as the future cardiac event. Especially the finding that feelings of exhaustion are a relatively short-term risk indicator for myocardial infarction raises the question whether vital exhaustion is a marker of subclinical heart disease.

\subsubsection{Case-control studies.}

One of the more extended case-control studies on the relationship between vital exhaustion and myocardial infarction was performed by Falger (1989). In this study 133 male myocardial infarction patients were compared with 133 neighborhoodcontrols and 192 hospital-controls. The second reference group was included to control for the effects of hospitalization on the report of feelings of exhaustion. Subjects were divided into two groups on the basis of a score above or below the median of the MQ $(=16$; that is, 8 or more affirmative responses to the 21 questions). Making use of this cut-off point, Falger estimated the relative risk for first 
myocardial infarction associated with vital exhaustion as $7.35(95 \% \mathrm{CI}=4.30$ $13.30)$ with the neighborhood-controls and as $2.90(95 \% \mathrm{Cr}=1.70-4.95)$ with the hospital-controls as reference groups. All odds ratios were statistically highly significant which confirms that feelings of exhaustion are a risk indicator for myocardial infarction. The data indicate also that elevated exhaustion scores are observed in other groups of patients (i.e., the hospital-controls). Falger has given three interpretations of this finding: 1) Vital exhaustion is (also) a precursor of diseases other than coronary artery disease; 2) Recent hospitalization causes retrospective bias in both myocardial infarction-cases and hospital-controls; and 3) Symptoms (anginal or non=cardiac) are associated with one or more elements of vital exhaustion (e.g. pain, or sleep problems; Falger, 1989). Arguments pro and contra these interpretations will be presented next.

ad 1. The plausibility that vital exhaustion is a precursor of other somatic diseases than coronary artery disease depends in part on the pathogenesis of the disease considered. However, the Rotterdam Civil Servants Study did not reveal an association between feelings of exhaustion and cancer or gastrointestinal disease. It might be that disorders with a low prevalence are preceded by vital exhaustion but that these associations are not objectified by prospective epidemiological studies due to the lack of statistical power to detect such relationships.

ad 2. In order to gain insight into the extent of confounding by retrospective bias in the case-control study of Falger, the method proposed by Raphael was used (Raphael, 1987). This method holds that the estimated relative risk of vital exhaustion is computed while adjusting for recall-bias by using a "validity scale". This validity scale consists of those items which discriminated between myocardial infarction patients and the neighborhood controls (the study of Falger), but which did not have a predictive power in the prospective study (the Rotterdam Civil Servants Study). It was possible to do so because the study of Falger was started before the completion of the prospective study and included the full item pool (that is, the 37 items of the MQ-A and the 21 added items). Subjects were classified as exhausted, intermediate or not exhausted, according to their scores in the third, second, or first tertile of the distribution of the MQ scores in the controll group. Firstly, the odds ratios adjusted for age, smoking, and angina were computed. Next, the same analysis was repeated including a correction for the validity scale. The relative risk of a score in the second tertile decreased from 3.13 to 2.97 when adjusted for the validity scale (i.e., recall-bias) and for the third tertile the decrease was from 10.07 to 6.88 . It can be concluded that recall-bias leads to an overestimation of the premonitory condition of exhaustion, but that the association between vital exhaustion and myocardial infarction continues to be of importance if correction for this bias is applied. This conclusion is in line with experimental research. Croyle and Sande have shown that students confronted with a "positive" test of a non-existing disease reported more risk behaviors, of which they had been told that they contributed to the occurrence of the disease. This implies that confirmatory search resulted in an overestimation of the exposition to accepted risk 
factors. However, the recollection of any symptom experienced in the months preceding the "discovery" of the disease was only moderately influenced. The search for meaning after the confrontation with a new disease is less directed at premonitory symptoms but rather at risk behaviors which are supposed to be causal to the disease (Croyle \& Sande, 1988). The possible information-biases of the case-control design do not seem to outweigh the operational and financial advantages of this research strategy in studies relating vital exhaustion to myocardial infarction.

ad 3. The importance of anginal and other somatic complaints to the presence and severity of vital exhaustion was considered in a case-control study in women who suffered myocardial infarction. Participants of that study were 79 women hospitalized because of a first myocardial infarction (mean age $59.3 ; \pm 9.1$ ) and 90 women hospitalized in the departments of General Surgery and Orthopaedic Surgery of the same hospital (mean age $57.5 ; \pm 9.4$ ). The myocardial infarction cases reported significantly more symptoms of vital exhaustion than the controls $(t=2.02 ; p=0.03)$. On the basis of a median split of the MQ score, $63 \%$ of the cases and $39 \%$ of the controls were exhausted before admission $\left(\mathrm{Chi}^{2}=10.02 ; \mathrm{p}=0.001\right)$. Non-anginal pain was strongly associated with a higher MQ score $(t=5.21 ; \mathrm{p}<0.001)$. The estimated relative risk of vital exhaustion adjusted for age, hypertension, smoking, and nonanginal pain was $2.75(95 \% \mathrm{CI}=1.28-5.87)$. This finding indicates that the relationship between vital exhaustion and myocardial infarction may very well be influenced by complaints caused by the discomfort of somatic complaints, but that this can not account for the association between vital exhaustion and myocardial infarction (Appels et al., in press).

In conclusion, of the interpretations suggested by Falger (1989) to explain the increased exhaustion scores in individuals with a non-cardiac disease (i.e., the hospital-controls) it appears that retrospective bias was of influence, but could not account for the association between exhaustion and future myocardial infarction; the confounding related to somatic complaints requires further investigation but is unlikely to be a sufficient explanation for the relationship between feelings of exhaustion and future manifestations of coronary artery disease.

Because the case-control studies reported by Falger and colleagues $(1989 ; 1992)$ and the prospective study by Appels et al. (1987) were carried out in the Netherlands, a replication study was performed at the John Sealy Hospital, Galveston, Texas, U.S.A. in the department of Dr. C.D. Jenkins (Mendes de Leon, 1988). In that study, 22 patients with acute or progressive anginal complaints were compared with 44 controls with another acute non-cardiac medical condition (most often orthopaedic patients). The odds-ratio was $2.36(95 \% \mathrm{CI}=0.95-5.88 ; \mathrm{p}=0.07)$ which is in support of the relationship between vital exhaustion and manifest coronary artery disease (Appels \& Mendes de Leon, 1989).

Although the Maastricht Questionnaire has a predictive validity and discriminates well between coronary patients and controls, there are some theoretical problems which are related to the use of a questionnaire to assess a state such as vital exhaus- 
tion. Elevated scores on questionnaires that ask for somatic and psychological symptoms are often influenced by a general tendency to complain (e.g. Costa et al., 1985). This could have resulted in false positives. Another problem with the use of questionnaires is that the assessment cannot be corrected for if a subject misinterprets a question. Consequently, its use for the selection of subjects for laboratory or clinical investigations may be debatable. Therefore, a semi-structured interview has been developed for the use in non-epidemiological studies. The content of this interview consisting of 23 questions is derived from the $\mathrm{MQ}$, including items which were found to be associated with future heart disease in the prospective study. The interview was tested for its discriminating power in a case-control study by Meesters. Subjects were 80 males who were hospitalized because of first myocardial infarction and 167 neighborhood controls matched for age. It was found that the interview scores were significantly higher in cases than in controls $(p<0.01)$. Apart from this main-effect, there was also an interaction between vital exhaustion and age, indicating that the mean exhaustion score of younger myocardial infarction patients was the highest. An additional advantage of the interview is that it allows for a precise insight into the duration of the exhaustion before myocardial infarction, Meesters inquired about the onset of each item of the interview. It was found that in more than half of the exhausted patients with myocardial infarction the median duration of the affirmatively answered items was six months or less. If items were responded to positively in the control group, it appeared that the duration often exceeded twelve months. In addition, these complaints were frequently side-effects of a chronic disabling condition and did not reflect a decline in physical and psychollogical functioning which characterized most of the myocardial infarction patients (Meesters, in preparation).

To summarize, vital exhaustion appears to be an adequate label for the condition of undue tiredness and general malaise prior to myocardial infarction. Vital exhaustion can be assessed with the Maastricht Questionnaire and the Interview Vital Exhaustion. Using the questionnaire, it was shown that wital exhaustion was significantly related to future manifestations of coronary artery disease. This relationship is present in both men and women and also in other countries than the Netherlands. Furthermore, the association between vital exhaustion and future manifestations of coronary artery disease is most evident in the year prior to the cardiac event. The next section elaborates upon research that addresses the development of vital exhaustion.

\subsection{Psychosocial precursors of vital exhaustion.}

If vital exhaustion is conceived as an end-stage of prolonged psychological tension, predisposing personality characteristics and environmental factors with a negative impact may contribute to its emergence. The theoretical basis for this assumption is outlined in section 1.1.3. Referring to the work of Glass (1977) it was suggested that Type $A$ individuals are at increased risk of becoming exhausted because of their sustained efforts to reach high set goals. Several studies have addressed the interaction 
between Type A behavior and vital exhaustion as related to myocardial infarction. Other contributing factors in the development of vital exhaustion are found in environmental circumstances. A distinction is made between situations with a predominantly psychological consequence (e.g. loss of a beloved one), referred to as life events, and situations which probably have combined physical and psychological consequences leading to feelings of exhaustion (e.g. overwork, or sleep problems).

\subsubsection{Type A behavior and Hostility.}

The case-control study by Falger (1989; section 1.2.2.) revealed that vital exhaustion was positively associated with Type A behavior as assessed by the Structured Interview. The mean exhaustion score of Type A subjects was significantly higher than of Type $B$ subjects $(13.3 \pm 10.2$ and $9.9 \pm 9.7$, respectively; $t=3.51 ; p<0.001)$. The study by Meesters also showed a significant association between vital exhaustion and Type $\mathbf{A}$ behavior as assessed by the Structured Interview. In addition, the data of this study revealed a significant interaction between Type A behavior and vital exhaustion as related to myocardial infarction which is concordant with the prediction of Glass (1977). This is illustrated in Figure 1.1. that shows the standardized relative risks with the non-exhausted Type Bs as the reference group. Both studies show that the exhausted Type As have a substantially elevated risk of myocardial infarction in men.

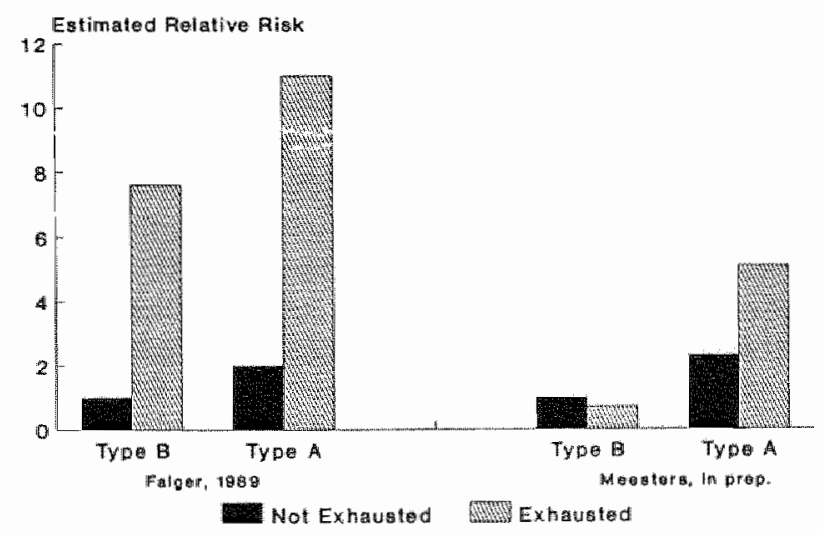

Figure 1.11. The risk of myocardial infarction as related to Type A behavior and vital exhaustion. The relative risks are computed with the "Vital Type B' as reference group.

The controversy about the validity of the studies relating Type A behavior to future coronary artery disease (e.g. Ragland \& Brand, 1988) stimulated the search of 'the toxic component' of this behavior pattern. A lot of attention has been given to hostility 
as a risk factor for myocardial infarction. Hostility, reflects a cynical mistrust of other people (Williams, 1987). Hostility can be assessed with the Cook-Medley Hostility Scale and the Buss-Durkey questionnaire. Both inventories were included in the casecontrol study of Meesters. The total-scores of both scales and on the sub-scales "assault", "indirect hostility", "irritation", and "resentment" of the Buss-Durkey were positively associated with vital exhaustion. When vital exhaustion and the separate hostility indices were simultaneously entered in a multivariate analysis, all hostility (sub)scales lost their discriminating power. This suggests that hostility is a risk factor either because it increases the risk to become exhausted, or because a cynical mistrust in other people is a concomitant phenomenon of an overall state of exhaustion (Meesters, in preparation).

\subsubsection{Life events.}

Another part of the case-control study by Falger $(1989 ; 1992)$ addressed the impact of several life-events on exhaustion and myocardial infarction. It was observed that a strong association existed between vital exhaustion and a number of stressful life events. In childhood these were: prolonged familial conflicts, prolonged financial problems, and unemployment of the father. Events in adult life that related to vital exhaustion were: serious conflicts with supervisors or subordinates, prolonged overtime work, serious educational problems with children, serious marital conflicts or conflicts with family members, death of a family member or beloved one, serious illness, and serious financial problems.

A similar pattern of associations was observed in the case-control study of female myocardial infarction patients. Positive associations were observed between vital exhaustion and adverse youth conditions such as poverty, parental unemployment, and conflicts at home. Feelings of exhaustion were also associated with a number of life events in adulthood, namely: serious conflicts at work with supervisors or subordinates, prolonged overtime work, serious educational problems with children, serious marital conflicts, ever been burned out or over-stressed, unwanted childlessness, and prolonged financial problems. There was no association found between vital exhaustion and the number of children.

It is of some importance to draw attention to the fact that "prolonged financial problems" increased the risk of myocardial infarction both in men and women. This is in line with the observation of Ruberman who reported that a major financial difficulty during the preceding year increases the risk of mortality after myocardial infarction (Ruberman et al., 1984).

\subsubsection{Overwork and lack of sleep.}

Feelings of exhaustion are not only caused by psychologically uncontrollable events or other forms of prolonged mental stress, but may also be caused by factors with 
additional physical consequences like double jobs or too many working hours, which deprives people from sufficient time spent relaxing (Falger et al., 1992). This issue is of importance, the more so in the presence of sleep problems. Falger showed that working overtime was related to both vital exhaustion and myocardial infarction. However, the association between overwork and myocardial infarction lost its discriminating power between cases and controls when vital exhaustion was controlled for. Thus, overwork appears to be a risk factor for manifest coronary disease especially if it results in a state of exhaustion.

Recent studies of job stress indicate that a combination of high demands and low control increases the risk of coronary artery disease. Moreover, people who work in such conditions are at risk to become exhausted (Karasek \& Theorell, 1990). This model was corroborated by Sihm in a study of young myocardial infarction patients. Results showed that the best single expression of the influence of quantitative job demands on coronary morbidity was obtained with the parameter "exhaustion after work", which assessed the impact of work on the daily life rather than merely time spent working (Sihm et al., 1991). This result is in line with the observation by Falger concerning overwork. Recently, Siegrist extended the model of Karasek and assumed that an imbalance between effort and experienced reward induces proneness for cardiac disease (Siegrist, 1991).

During the last decade several studies have indicated that sleep problems might belong to the risk indicators for coronary artery disease (Partimen et al., 1982; Siegrist et al., 1987; Koskenvuo et all, 1988). A detailed analysis of the follow-up data of the Rotterdam Civil Servants Study (Appels \& Schouten, 1991) showed that being exhausted on waking up was predictive of future myocardial infarction (relative risk $=2.1$ ). Interestingly, the relative risk of waking up exhausted increased to 2.6 when subjects who had problems falling or staying asleep were excluded from the analysis. This makes it likely that early morning tiredness predicts future myocardial infarction even without the contribution of problems falling or staying asleep. In passing, it is of some interest to note that Koskenvuo observed a positive association between hostility and morning fatigue (Koskenvuo, 1988).

Van Diest carried out a series of studies on the association between sleep complaints and sleep disorders on the one hand and vital exhaustion on the other hand. It was observed that sleep complaints were more prevalent among exhausted subjects (van Diest, 1990). Of interest are the results of a recent sleep physiological study, in which it was shown that sleep latency and the sleep duration of exhausted and non-exhausted subjects were only marginally different. The major difference was a significantly reduced delta-sleep among the exhausted subjects. Most normal recovery processes are supposed to take place during this delta-sleep or deep-sleep phase (van Diest, in press). It may be inferred from that study that exhausted persons do not spend less time sleeping, but that the restorative properties of the sleep are less efficient. 


\subsection{Vital exhaustion and other tiredness-related constructs.}

Tiredness is observed in numerous physical and psychiatric disorders. This review will be restricted to the most obvious conditions that may confound the valid assessment of vital exhaustion since it is beyond the scope of the present chapter to discuss the complete issue of fatigue in extenso. The syndromes that will be addressed are: depression, burnout, and the chronic fatigue syndrome. In all these conditions, fatigue plays a crucial role in the diagnosis and the differences and congruences with vital exhaustion will be pointed out.

\subsubsection{Depression.}

The MQ includes some known characteristics of depression, such as listlessness, fatigue, loss of libido, and waking up exhausted. In fact nine of the 23 questions of the exhaustion interview are identical to the questions used to assess depression according to the DSM-III-R criteria. This raises the question whether vital exhaustion is conceptually different from depression.

Estimates of the prevalence of depression among myocardial infarction patients vary between $20 \%$ and $45 \%$. Approximately half of these depressions are already present before myocardial infarction and the other half can be considered to be mainly reactive (Lloyd \& Cawley, 1983; Schleifer et al., 1989). Major depressive disorders assessed by the Diagnostic Interview Schedule according to DSM-III-R criteria (Robins et al., 1982) have been found to be related to future cardiac events in patients with coronary heart disease, while statistically controlling for the severity of coronary disease, cardiac pump function, and smoking status (Carney et al., 1988). Myocardial infarction patients who are depressed have a poorer prognosis (i.e., cardiac death, cardiac arrest, re-infarction) than patients who are not depressed (Silverstone, 1987; Ladwig et al., 1991). Shekelle and Ostfeld found in a prospective study that nonsurvivors of myocardial infarction had a higher score on the depression scale of the Minnesota Multiphasic Personality Inventory than survivors did (Shekelle \& Ostfeld, 1965). Hällström observed that depression was associated with cardiac disease in women (i.e., angina, not with myocardial infarction or cardiac death). On the basis of a meta-analysis of these and other studies Booth-Kewley and Friedman concluded that depression is reliably associated with future manifestations of coronary disease (BoothKewley \& Friedman, 1987). However, this conclusion is questioned by Matthews after re-analysis of the data with the exclusion of all case-control studies (Matthews, 1.988). A recent study by Anda gave additional support for the association between depression and coronary disease (Anda et al., 1993).

There are two indications that vital exhaustion differs from depression. ltems asking for a lowered self esteem or guilt feelings were not predictive for myocardial infarction in the Rotterdam Civil Servants Study. In addition, coronary patients most often blame others for the occurrence of negative events, although they confirm that the 
event made them feel helpless at the time it occurred (Byme, 1980); low selfesteem and self-reproach are rare among coronary patients. Consequently, the core elements of depression, as described by cognitive psychology (I am the cause of my misfortune; I will always be a failure or have misfortune; whatever I do is unsuccessful), are rarely heard among patients with coronary artery disease.

The strongest argument to distinguish exhaustion from depression is provided in a study by van Diest and Appels (1991). The Profile of Mood States was used to monitor feelings of a depressed mood, vigor, and fatigue in $\mathbb{2} 2$ interview-defined exhausted and 10 non-exhausted healthy males during an observation period of three weeks. Current affective, cognitive, motivational, and somatic symptoms of depression were further assessed with the Beck Depression Inventory. Significant differences between exhausted and non-exhausted subjects were observed with regard to loss of vigor and fatigue. A depressed mood, the key symptom of depressive disorders, was hardly ever mentioned by the exhausted individuals during the three weeks of observation. Both groups differed significantly with regard to their mean Beck depression scores. However, a detailed analysis of the symptoms showed that this difference could be exclusively attributed to the symptoms "fatigue", "work inhibition", "sleep disturbances", and "loss of libido". Only one subject of the exhausted group reported a depressed mood, while none of the exhausted subjects reported weight loss, loss of appetite, suicidal ideation, self accusation, or a sense of punishment. Studies which used the Profile of Mood States to register the moods of coronary patients stress the importance of distinguishing a depressed or sad mood from feelings of fatigue and lack of vigor. Shephard observed that coronary patients had significantly lower vigor scores as compared to a control group. The mean scores of the depression scale did not differ between both groups (Shephard et al., 1985). Dimsdalle observed that fatigue and not depression as assessed by the Profile of Mood States predicted cardiac events (hospitalization, myocardial infarction, resuscitation, or death) in the year after catheterization (Dimsdale et all., 1981 ).

In sum, the answer to the question whether vital exhaustion is distinct from depression depends at least partially on the relative weight that is given to the mood disturbances in the assessment of depression. Loss of interest or pleasure is common but a depressed mood is rare among exhausted individuals. Almost all depressed subjects satisfy the criteria for exhaustion, while about half of the exhausted subjects have symptoms that are necessary for the diagnosis of depression. The studies that showed a relationship between exhaustion and future cardiac events do not contradict that a depressive episode precedes the onset of the acute coronary syndromes. However, this conception of the psychological precursors of myocardial infarction is too imprecise because it incorrectly suggests that feelings of sadness precede myocardial infarction while it does not stress the relative importance of mental and emotional exhaustion. 


\subsubsection{Burnout.}

The concept of burnout was first mentioned by Freudenberger in order to describe the job-related physical and mental state that was observed among initially enthusiastic, young volunteers who worked in a drug clinic. After one year, many of them felt exhausted, easily irritated, and had developed a cynical attitude towards the clients and

a tendency to avoid them (Freudenberger, 1974). This origin of the concept has influenced the direction of the research on burnout. From the beginning on, two theoretical positions were debated. On the one side it was remarked that burnout symptoms can also be observed among persons who do not have a job at all (e.g., Pines \& Aronson, 1988), while on the other side arguments were put forward that burnout occurs only among individuals who do "people work". Maslach, for example, belongs to those who reserve burnout for persons who continuously work with other people and defines it as "a syndrome of emotional exhaustion, depersonalization, and reduced personal accomplishment that can occur among individuals who do people work of some kind" (Maslach \& Johnson, 1986). Pines and Aronson (1988) define burnoul as "a state of physical, emotional and mental exhaustion caused by long-term involvement in situations that are emotionally demanding" .

In an attempt to investigate whether burnout is best represented by one or three dimension, Shirom made a review of all available validation studies. It was found that of the three sub-scalles of the Maslach Burnout Inventory the emotional exhaustion scale correlated best with work-related variables and with observations made by spouses and superiors: "The major conclusion which may be drawn from the validation efforts is that the unique content of burnout has to do with a depletion of an individual's energetic resources". Consequently, Shirom defines burnout as "a combination of physical fatigue, emotional exhaustion and cognitive weariness" (Shirom, 1989).

It is evident that burnout has much overlap with the concept of vital exhaustion. Six of the nine items which form the emotional exhaustion sub-scale of the Maslach inventory are part of the MQ. The major difference is that increased irritability is considered to be an element of vital exhaustion, while Maslach uses peer ratings of irritability as an external criterium for the validation of the inventory. Another distinction is that the time frame of symptomatology is taken into account in the assessment of exhaustion (section 1.2.2.) which is not of relevance for burnout.

The clinical interviews that were carried out to develop the $M Q$ revealed that one or more episodes of burnout preceded the current condition of exhaustion. Patients often reported that the mental state prior to admission was very similar to the earlier period when they were burned out. For that reason the item "have you ever been burned out?" was added to the inventory that was used in the Rotterdam Civil Servants Study. An affirmative answer was associated with an increased risk of future myocardial infarction (age adjusted relative risk $=1.31 ; 95 \% \mathrm{CI}=1.10-2.99$ ). A positive reply was also strongly correlated with the MQ score, suggesting not only that burnout and 
vital exhaustion share common variance, but also that an earlier period of breakdown in adaptation to stress was associated with the later condition of exhaustion at screening (Appels, 1991). This finding corroborates with the observation of Paffenbarger that students who had experienced a period of exhaustion were at increased risk for future coronary disease (Paffenbarger et al., 1966).

\subsubsection{The Chronic Fatigue Syndrome.}

Persistent and excessive fatiguability is the key characteristic of the Chronic Fatigue Syndrome, Co-occurring symptoms include: painful joints and muscles, headaches, sleep problems, painful lymph nodes, cognitive problems, emotional instability, and depression. The Chronic Fatigue Syndrome seems to be virtually the same as Myalgic Encephalomyelitis. The supposed viral etiology (Epstein-Barr virus infection) has never been unequivocally objectified. Therefore, Holmes and colleagues (1988) composed a consensus definition of the Chronic Fatigue Syndrome. Briefly, it is states that the Chronic Fatigue Syndrome is present in case of "new onset of persistent or relapsing, debilitating fatigue or easy fatiguability in a person who has no previous history of similar symptoms, that does not resolve with bed-rest, and that is severe enough to reduce or impair average daily activity below $50 \%$ of the patient's premorbid activity level for a period of at least six months". In addition, a set of specified symptom criteria are suggested. It appears that psychiatric disorders are prevalent among individuals with the Chronic Fatigue Syndrome (e.g., Wessely \& Powell, 1989; Manu et al., 1988). The estimates of concomitant depressive disorder range from 40 to $70 \%$. Moreover, the psychological component of fatigue complaints increases substantially if the duration exceeds four months (Morrison, 1980). Presently, it appears preferable not to use a psychiatric label for the Chronic Fatigue Syndrome because a viral cause is not completely excluded and, in addition, the Chronic Fatigue Syndrome may refer to a heterogeneous group with fatigue complaints (Ray, 1991).

Fatigue is observed as a prevalent complaint in the general population. For example, $10 \%$ of all patients in a general practice reported interfering fatigue complaints with a duration of more than one month (David et al., 1990). The study of David and colleagues did not show differences in gender, age, or occupation in subjects with or without fatigue complaints. Those who attributed fatigue to a physical cause had higher fatigue scores than subjects who explained their fatigue as a consequence of family, emotional, or social problems, or poor sleep. It is of interest to note that only one patient fulfilled the criteria for the Chronic Fatigue Syndrome.

In sum, vital exhaustion and the chronic fatigue syndrome share excessive tiredness as a common feature but the constructs appear to differ in their clinical presentation. The difference and similarities between the Chronic Fatigue Syndrome and vital exhaustion require further research. Both constructs have the difficulty that fatigue is correlated with measures of depression and anxiety (Montgomery, 1983). Therefore, it 
is likely that instruments that evaluate the Chronic Fatigue Syndrome correlate highly with assessments of vital exhaustion. There are a few apparent differences between vital exhaustion and the Chronic Fatigue Syndrome which could be further investigated. Firstly, in most people with the Chronic Fatigue the fatigue is often contingent upon physical or mental exertion, whereas the tiredness in vital exhaustion seems constantly present, most often already at the time of waking up. Secondly, in the general population the Chronic Fatigue Syndrome is encountered more frequently in women and in higher educated individuals, while vital exhaustion is most likely to be prevalent in men with a lower education. Thirdly, the importance of the duration of fatigue complaints could be an important area of future studies.

\subsection{Research questions.}

The previous review gives rise to numerous questions as to the etiology of vital exhaustion, its discriminative validity with regard to other fatigue-related syndromes, but most importantly, the validity of the predictive association between vital exhaustion and future cardiac events. This will be the main issue of the following chapters. The rational for the patient selection, methodology, and the details of the procedures will be described in Chapter 2. Below, a short outline will be presented of the hypotheses under investigation.

Currently there are several plausible hypotheses that explain the tiredness before myocardial infarction (sections 1.1.1. and 1.2.1.). If the hypothesis that vital exhaustion is explained by underlying coronary artery disease is falsified and, in addition, confirmation is found for an independent predictive value of vital exhaustion in the clinical course of coronary artery disease, then credibility is obtained for the belief that vital exhaustion prior to myocardial infarction requires a psychological interpretation. More specifically the following hypotheses are tested:

Hypothesis 1: Vital exhaustion is related to the extent of coronary artery disease. (Chapter 3).

Hypothesis 2: Vital exhaustion is related to a poor pump function of the heart in patients with coronary artery disease. (Chapter 3).

The advantage of research designs that use angiography data is that they allow for a direct investigation of the underlying atherosclerotic disease. However, cross-sectional studies like the one that will be described in chapter 3 carry problems of causal inference. Therefore, a sub-sample of the coronary angiography patients was used in which the impaired coronary perfusion was restored by Percutaneous Transluminal Coronary Angioplasty (PTCA). During the PTCA procedure a balloon is placed at the site of the coronary stenosis and is subsequently inflated. By this procedure the narrowing in the coronary artery is dilated which results in a restoration of the 
coronary blood perfusion. If vital exhaustion is merely a consequence of impaired coronary blood supply, successful PTCA should be followed by a substantial reduction of feelings of exhaustion. However, if vital exhaustion is marginally affected by successful PTCA, impaired coronary perfusion can not account for the presence of vital exhaustion. This has lead to the following hypothesis:

Hypothesis 3: Restoration of coronary perfusion by successful coronary angioplasty is followed by a substantial reduction of viral exhaustion. (Chapter 4).

If the previous three hypotheses will be confirmed there is little need for alternative (i.c., psychological) explanations for the tiredness prior to myocardial infarction. But if the hypotheses are falsified, then there is room for alternative explanations of the premonitory feelings of exhaustion.

Next, it was investigated whether the predictive value of vital exhaustion for myocardial infarction which was shown in the Rotterdam Civil Servants Study (section 1.2.1.) could be replicated while explicitly controlling for the underlying coronary artery disease. For this purpose, the above mentioned group of consecutive PTCA patients were followed up during 1.5 years. New manifestations of coronary artery disease are known to occur in $20-30 \%$ of all patients after an initially successful PTCA within a year. This relatively high incidence of new cardiac events is assumed to be mainly caused by restenosis of the dilated lesion. It was investigated whether vital exhaustion as assessed two weeks after successful PTCA was related to new cardiac events during the follow-up period of 1.5 years and if this association was independent of the extent of atherosclerosis and other known predictors of the clinical course after PTCA. The hypothesis tested is:

Hypothesis 4: Vital exhaustion predicts new cardiac events after successful coronary angioplasty, independenty of the extent of underlying coronary artery disease and other predictors of the clinical course affer angioplasty. (Chapter 5).

If the extent of atherosclerosis is found not to be associated with vital exhaustion, then other physiological mechanisms can be thought of to explain the relationship between vital exhaustion and future manifestations of coronary artery disease. One important area of research on acute coronary syndromes is the development of the blood-clot (thrombus). A thrombus may (partially) occlude a coronary artery and hence result in acute myocardial infarction or unstable angina pectoris (e.g., Fuster et al., 1992). This lead to an investigation on the relationship between vital exhaustion and factors that are of relevance to the clotting process (i.e., coagulation and fibrinolysis). For this study a different sample of apparently healthy men was recruited in order to test the following hypothesis:

Hypothesis 5: Vital exhaustion is associated with coagulation and fibrinolytic factors that promote clot-formation and stabilization (Chapter 6).

In Chapter 7 the findings of chapters three to six are discussed in relation to the response to injury model of progressive coronary artery clisease. This results in a new psychobiological model that can account for the relationship between vital exhaustion and future cardiac events. 


\section{Chapter 2.}

\section{Methods and Rational.}

The present chapter deals with the procedures of the recruitment of the subjects and the applied measurements. In a broad sense, two lines of investigations were carried out: a) on the relationship between exhaustion and coronary artery disease in patients referred for coronary angiography; b) on the association between vital exhaustion and blood-clotting factors in healthy subjects. For each hypothesis under consideration a description of the recruitment of the subjects will be given including the adhered exclusion criteria. Thereafter, the measurements will be presented which is followed by the justification of the statistical procedures. The studies were performed after approval of the medical ethical committee of the University Hospital Maastricht.

\subsection{Vital exhaustion in cardiological patients.}

The following four hypotheses will be tested in a sample of consecutive cardiological patients referred for coronary angiography or coronary angioplasty:

Hypothesis 1: Vital exhaustion is related to the extent of coronary artery disease.

Hypothesis 2: Vital exhaustion is related to a poor pump function of the heart in patients with coronary artery disease.

Hypothesis 3: Restoration of coronary perfusion by successful coronary angioplasty is followed by a substantial reduction of vital exhaustion.

Hypothesis 4: Vital exhaustion predicts new cardiac events after successful coronary angioplasty, independently of the extent of underlying coronary artery disease and other predictors of the clinical course after angioplasty.

The first two hypotheses were investigated with a cross-sectional design in order to examine if exhaustion in patients with coronary artery disease is caused by a poor coronary blood-supply to the heart or a reduced cardiac pump function. The third hypothesis contains also a test of the proposition that exhaustion is contingent upon 
impaired coronary perfusion, but in this case a quasi experimental design is used. Making use of a prospective design hypothesis 4 tests if vital exhaustion is an independent predictor of the progression of coronary disease.

\subsubsection{Hypothesis 1 .}

The Rotterdam Civil Servants Study revealed that vital exhaustion is a short-term precursor of myocardial infarction (Appels \& Mulder, 1988). This observation would not require a psychological interpretation if exhaustion prior to myocardial infarction was the result of underlying coronary artery disease. The rational for this objection is valid since the Rotterdam Civil Servants Study made use of the resting electrocardiogram and the Rose Questionnaire (Rose et al, 1982) to exclude the presence of manifest coronary artery disease at screening. This procedure does not guarantee that patients with (subclinical) coronary artery disease were unintentionally included. In this respect it is of relevance to note that $70-90 \%$ of the episodes of myocardial ischemia are silent, that is, they occur without accompanying anginal pain (Cohn, 1989; Kellerman \& Braunwald, 1990). As was already mentioned in the previous chapter, the presence of subclinical coronary artery disease at screening may have accounted for both the presence of vital exhaustion at the time of entry to the study as well as the development of future myocardial infarction. In other words, underlying subclinical coronary disease may have confounded the relationship between vital exhaustion and future myocardial infaretion.

If this objection is correct, then one would expect a relationship between the extent of underlying coronary artery disease and the severity vital exhaustion. On the other hand, the argument of confounding by coronary artery disease becomes less likely if it is found that the association between coronary artery disease and vital exhaustion is absent or very weak. This is the background of the present attempt falsify or confirm hypothesis 1: Vital exhaustion is related to the extent of coronary artery disease. This hypothesis is tested in a cross-sectional design relating the extent of coronary artery disease as assessed during coronary angiography to vital exhaustion as measured with the Maastricht Questionnaire.

Patients. Consecutive patients who underwent diagnostic coronary angiography at the department of Cardiology, University Hospital of Maastricht were asked to participate in a study on "the relationship between emotions and diseases" between august 1988 and december 1989. To be referred for coronary angiography, patients had to suffer anginal symptoms and show electrocardiographical signs suggestive of coronary artery disease, either spontaneously or during exercise testing. The diagnosis on admission was either stable angina, unstable angina, or myocardial infarction. Patients were not included if diagnostic angiography was performed in the setting of an electrophysiological study for cardiac rhythm disturbances. Three exclusion criteria were applied: age $>70$ years, previous revascularization by coronary bypass surgery or coronary angioplasty, severe co-morbidity (e.g., cancer, severe renal disease, 
valvular disease, psychiatric disorder). The age criterium was introduced because the validity of the assessment of vital exhaustion has as yet not been investigated in the older aged and it is imaginable that physical immobility, cognitive impairment, and liwing in a nursing home may confound the assessment of feelings of exhaustion. The reason to exclude patients who had a previous revascularization was that these treatments might complicate the determination of the severity of coronary artery disease. The exclusion of patients with severe co-morbidity was applied in order to prevent confounding of the assessment of vital exhaustion by a disabling condition. After approval of the nursing staff considering whether the patient's clinical condition allowed a request for participation in the study, written informed consent was asked by the investigator.

In total, 322 eligible patients were approached; 6 refused to participate and 9 filled out an invalid questionnaire. This resulted in 307 patients of whom the demographic and diagnostic characteristics are described in chapter 3.

Methods. Vital exhaustion was assessed using the Maastricht Questionnaire (MQ). This 21 -item questionnaire has a score range from 0 to 42 . Appels and colleagues (1987, 1988) observed in the Rotterdam Civil Servants Study a mean MQ score of 8.8 \pm 8.7 in healthy men $(N=3877)$. Falger (1989) found approximately the same mean MQ score in a healthy control group $(9.0 \pm 9.7 ; \mathrm{N}=133)$, while the hospital controls had slightly higher scores (11.7 $\pm 9.8 ; N=192)$ and patients with myocardial infarction had significantly elevated $M Q$ scores $(18.0 \pm 10.8 ; \mathrm{N}=133$ ). In another study, Falger et al. (1992) showed that for women the MQ scores tend to be overall elevated. In hospitalized subjects without cardiac disease the mean MQ was $17.0 \pm 11.2(\mathrm{~N}=79)$ and in myocardial infarction patients $20.6 \pm 11.9(\mathrm{~N}=90)$. Patients were asked to report about their feelings during the three months preceding the current hospitalization. Whenever possible, patients filled out the questionnaire before catheterization to prevent biases due to knowledge of the results of the angiography in the assessment of exhaustion.

Coronary angiography was used to investigate the extent of coronary artery disease. In brief, the procedure is as follows: A sheath is introduced into the groin and the femoral artery (Judkins technique) or into the elbow and the arteria brachialis (Sones technique). Thereafter, intravenous heparin is given (100 U/ $/ \mathrm{kg}$ body weight) in order to prevent thrombo-embolic complications. Subsequently, a catheter is forwarded into the ascending aorta. A hole at the end of the catheter allows the selective injection of contrast fluid into the left or the right coronary system. While the contrast fluid is injected, a film is made to visualize the coronary arteries. Several projections are taken to guarantee optimal evaluation. Following coronary angiography, the left ventricular angiography is performed in two projections $\left(30^{\circ}\right.$ right anterior oblique and $60^{\circ}$ left anterior oblique). At the end of the procedure protamine (5000 U intravenously) is given. Thereafter, the catheter and sheath are removed and the puncture site is manually compressed until bleeding stops. The routine procedure in the department of Cardiology is that the films are evaluated by a team of experienced 
cardiologists to assess the location and severity of the coronary stenoses. As a measure of severity of coronary stenosis six categories are used: no lesion, and lesions $<50$ $\%, 50-70 \%, 70-90 \%, 90-99 \%$, and $100 \%$. The location of the coronary lesion is categorized to be in one of the three major coronary arteries, i.e., right coronary artery (RCA), left anterior descending artery (LAD), and the circumflex artery (CX). RCA stenoses include: the proximal, mid, or distal RCA, and the posterior descending branch. LAD stenoses include: the proximal, mid, or distal LAD, and the diagonal branches. CX lesions include: the proximal or mid/distal CX, the marginal branch, or the posteral descending branch. Stenoses in the left main stem are mentioned separately because of the diagnostic importance of these types of lesions.

In the present study the 'severity of coronary artery disease' indicates the percentage of narrowing at the site of the most severe lesion, while the number of diseased vessels is indicated by 'the extent of coronary artery disease' (i.e., the RCA, LAD, or CX). Lesions of less than $50 \%$ were considered as clinically non-significant. In addition, a few other, more subtle, procedures were used (e.g., the total number of lesions $>50 \%$, or the attribution of extra weight to a lesion in the left main stem); these procedures will be described when relevant in the coming chapters.

Statistical analysis. Analysis of variance was used to test if patients with more severe coronary artery disease had increased feelings of exhaustion; the MQ score was used as dependent variable and the categories of the extent of coronary artery disease as independent between-subjects factor. The power to detect a difference of half a standard deviation of the MQ scores, with 300 subjects and $\alpha=0.05$ is $>90 \%$ (Cohen, 1988). In addition, regression analysis is performed to estimate the amount of explained variance of $\mathrm{MQ}$ scores by the extent of coronary artery disease. The results are reported in chapter 3 .

\subsubsection{Hypothesis 2 .}

In chapter 1, section 1.1. it was mentioned that a poor pump function of the heart may result in feelings of tiredness. To investigate whether this suggestion can explain the presence of exhaustion in patients with coronary artery disease, the following hypothesis was tested: Vital exhaustion is related to a poor pump function of the heart in patients with cononary artery disease. If this hypothesis is confirmed then there will be no need for alternative explanations for exhaustion, but if the hypothesis is falsified, then there is room for other factors that may account for feelings exhaustion in coronary patients.

Patients. left ventricular ejection fraction was calculated from the left ventricular angiograms. The 307 patients who were described in the previous section were, in principle, eligible to test the relationship between the pump function of the heart and the severity of vital exhaustion.

Methods. The assessment of vital exhaustion has already been described above (section 2.1.1.). The two usual procedures to obtain information about the left 
ventricular function are angiography and echocardiography of which the former procedure will be used here. The pump function of the heart was assessed by calculating the left ventricular ejection fraction using the modified Stanford needle adapted for biplane calculation (Bär et al., 1984). In short, the procedure is as follows: A catheter is forwarded into the ascending aorta in a way analogous to coronary angiography and moved through the aortic valve to the apex of the left ventricle. Then, contrast fluid is injected and the motion of the left ventricular contour is registered on film from two projections $\left(30^{\circ}\right.$ right anterior oblique and $60^{\circ}$ left anterior oblique). From the film the end-diastolic volume and the end-systolic volume are derived, that is, when the left ventricle is maximally filled c.q. dilated or minimally filled c.q. constricted, respectively. Next, the difference is calculated between the end-diastolic and end-systolic volume, i.e., the stroke volume. The stroke volume is a relatively constant fraction of the end-diastolic volume (normally $65-70 \%$ ) which is referred to as the left ventricular ejection fraction.

For the present study, three categories of left ventricular ejection fraction were used on the basis of clinical relevance: $\leq 40 \%$ (poor), $40-60 \%$ (moderate), and $>60$ $\%$ (good). Patients with coronary disease but without a history of myocardial infarction do rarely have an enlarged ventricle nor a reduced ejection fraction (less than 10 $\%$ of the cases). On the other hand, in case of coronary disease with myocardial infarction a reduced ejection fraction is observed in about half of the patients.

Statistical analysis. The three categories of the left ventricular function were used as the between-subjects factor in an analysis of variance with the MQ score as dependent variable. In addition, regression analysis was applied with the $\mathrm{MQ}$ as dependent and the categorized ejection fraction as predictor variable. It was decided not to use the continuous ejection fraction values because one would expect much uninterpretable variance in the upper range (e.g. $>60 \%$ ) of the ejection fraction, due to the expected low prevalence of a poor pump function in the present sample. The results of this analysis are presented in chapter 3.

\subsubsection{Hypothesis 3 .}

The purpose of the previous hypotheses was to evaluate the relationship between vital exhaustion and measures of coronary heart disease with a cross-sectional design. This methodology causes uncertainty as to the causal inference. Therefore, it was decided to apply a more experimental design to investigate the relationship between impaired coronary perfusion caused by coronary artery disease and vital exhaustion. In a subsample of the 307 coronary angiography patients described above, impaired coronary perfusion was treated with Percutaneous Transluminal Coronary Angioplasty (PTCA). The basis of this procedure was first described by Dotter (1964) who dilated a stenosis in the popliteal artery (the artery in the hollow of the knee) to treat perfusion disturbances of the lower part of the leg. Grünzig was able to perform the first percutaneous transluminal angioplasty in the coronary artery in 1977. It was shown that coronary 
angioplasty resulted in an effective restoration of coronary perfusion (Grünzig, Senning \& Siegenthaler, 1979). Presently, PTCA is successful in $90-95 \%$ of all cases and it results in a disappearance of anginal complaints. Although the use of PTCA was initially limited to patients with stable angina and single vessel disease, nowadays the application of PTCA is extended to patients with multi-vessel disease and also in diagnoses of unstable angina or acute myocardial infarction.

If exhaustion is the result of impaired bllood supply to the heart as a consequence of coronary artery disease, then, successful restoration of coronary perfusion by PTCA should be followed by a substantial reduction of feelings of exhaustion. Conversely, if vital exhaustion is not markedly influenced by successful PTCA, then impaired coronary perfusion can not account for vital exhaustion. This has lead to the formulation of hypothesis 3: Restoration of coronary perfusion by successful coronary angioplasty is followed by a substantial reduction of vital exhaustion. For this purpose vital exhaustion was assessed before PTCA, two weeks after discharge, and again after six months.

Patients. In addition to the exclusion criteria specified in section 2.1.1. it was required that 1) patients were symptomatic on admission (angina class II-IV), 2) at least one coronary stenosis was present of $\geq 70 \%, 3$ ) PTCA was successful, and 4) the patient was not admitted with a diagnosis of acute or recent $(<1$ month) myocardial infarction. The first two additional criteria reflect the usual referral strategy for PTCA. The inclusion of patients with a successful PTCA only was inherent in the hypothesis under investigation, which is based on successful restoration of coronary perfusion by PTCA. Patients with acute or recent myocardial infarction were excluded because the consequences of myocardial damage could confound the assessment of vital exhaustion before versus after PTCA.

The University Hospital Maastricht is a referral clinic for the region of the Middle and the Southern part of Limburg, the Netherlands. Approximately $60 \%$ of the patients received their usual cardiological care in one of the regionall hospitals. PTCA was performed in 196 patients, of whom 120 fulfilled the inclusion criteria. The details of the patient selection are presented in chapter 4 .

Methods. Vital exhaustion was assessed three times, making use of the Maastricht Questionnaire: before PTCA, two weeks after discharge from the hospital, and six months after PTCA. Questionnaires were not used if returned more than two weeks too late or if more than five items of the MQ were not answered. The follow-up assessment of exhaustion was complete in 108 patients (90\%) at two weeks, and in 85 (75\%) after six months. Non-response to the questionnaire was not related to any of the clinical or demographic characteristics, nor to the initial MQ score before PTCA.

PTCA was performed following the standard procedure which briefly holds the following: A guiding catheter is introduced in the coronary system in a similar way as the catheter introduction for coronary angiography (see section 2.1.1.). Then, a floppy wire is advanced into the diseased artery. After crossing the culprit lesion this wire is further advanced in the distal part of the coronary artery. Thereafter, a dilating 
catheter is advanced over the wire until it is positioned at the middle of the lesion. This dilating catheter has a small balloon (length $2.0 \mathrm{~cm}$ ) at the tip. Next, the balloon is inflated which results in a temporary $(60-180 \mathrm{~s})$ obstruction of the coronary vessel. In general, this leads to myocardial ischemia after $30 \mathrm{~s}$ since the muscle cells have a restricted reserve of adenosine-triphosphate which suffices for approximately half a minute. In some patients no ischemia occurs because of collateral circulation. PTCA is judged to be successful when a reduction of the stenosis to less than $50 \%$ of the diameter of the coronary vessel is obtained (= the residual stenosis) in combination with good coronary flow. It has been shown that the atherosclerotic lesion is compressed during PTCA and for the most part pushed into the arterial wall (see e.g., Liu et al., 1989; and chapter 7). The final result is that the balloon inflation results in a diminished coronary narrowing and a subsequent restoration of the coronary perfusion of the segments behind the dilated lesion. PTCA is repeated with higher pressures, longer inflation periods, or larger balloons in case of insufficient results. Before removing the wire and balloon catheter a waiting period of 5 to 10 minutes is applied in order to determine the development of recoil of the dilated lesion. This can occur due to the elasticity of the vessel wall, to deterioration of a dissection, or to thrombus formation. In such cases repeat-PTCA is performed during the same procedure until a satisfactory result is reached.

Statistical analysis. Univariate associations between the clinical variables and vital exhaustion were examined in this sub-sample in the same way as described in section 2.1.1. The short-term effect of PTCA on exhaustion was evaluated by means of a paired t-test. In addition, the number of exhausted patients was assessed making use of MQ cut-off points based on the tertiles of the MQ distribution in the general population. The contribution of the stenoses that were not dilated during PTCA - which could occur in case of multiple lesions $>50 \%$ - to the severity of exhaustion at two weeks follow-up was examined by analysis of variance. The number of vessels with an unapproached or residual stenosis $>50 \%$ was used as between-subjects factor and the MQ score as dependent variable.

To examine the predictors of change in MQ scores from before PTCA to two weeks after discharge, a difference score between both MQ scores was calculated. A multiple regression analysis was carried out to investigate the predictors of the change in $\mathrm{MQ}$ scores.

The long-term (six months) effect of PTCA was investigated in patients who had an event-free follow-up, that is, no myocardial infarction, coronary bypass surgery, or repeat PTCA. The three assessments of exhaustion were entered as a within-subject factor. The effect of recurrent typical anginal complaints was examined simultaneously as between-subjects factor. This resulted a $3 \times 2$ analysis of variance with the MQ score as dependent variable. The results are presented in chapter 4. 


\subsubsection{Hypothesis 4.}

It was shown in the prospective Rotterdam Civil Servants Study (Appels et al,, 1987; 1988) that vital exhaustion was of predictive value for future myocardial infarction (see chapter 1, section 2.1.). This finding was supported by another study (Appels \& Otten, 1992) where the feeling of being exhausted at the end of the day was associated with an increased relative risk of myocardial infarction. However, as mentioned above, both studies may have been confounded by underlying coronary artery disease. Therefore, the present study was conducted to investigate the predictive value of vital exhaustion for future cardiac events while explicitly controlling for the underlying coronary artery disease.

For that purpose, the occurrence of new cardiac events was investigated in patients who underwent successful PTCA. The advantage of this model is twofold. Firstly, in patients who have coronary angioplasty, a precise evaluation of the coronary arteries is obtained before and after dilatation. This allows a prospective study where the relationship between vital exhaustion and future cardiac events is investigated with the extent of underlying coronary artery disease as a control variable. Secondly, the incidence of new cardiac events after PTCA is between 20 and $30 \%$ within a year after the initially successful treatment (Detre et al., 1989; Rupprecht et al., 1990; Serruys et al., 1990). This high incidence brings about that in order to predict new cardiac events from a certain exposure variable (i.c., vital exhaustion) the sample size of a prospective study can be kept at a relatively modest magnitude.

The most important explanation for the high incidence of new cardiac events after successful PTCA is "restenosis" of the dilated lesion. In section 2.1.4. it was already mentioned that during PTCA the coronary lesion is more or less pushed into the arterial wall. This causes damage to the intimal layer and the media resulting in direct exposure of collagen to the blood. This promotes several kinds of growth factors and platelet activation factors which subsequently result in smooth muscle cell proliferation and increased platelet adhesion at the site of dilatation (e.g. Serruys et al., 1988; Liu et al., 1989; Bergtson et al., 1990). More details of the process of restenosis after PTCA are discussed in chapter 7.

In chapter 5 an attempt is made to replicate the Rotterdam Civil Servants Study, while controlling for the severity of underlying coronary artery disease. This is formulated in hypothesis 4: Vital exhaustion predicts new cardiac events after successful coronary angioplasty, independently of the extent of underlying coronary artery disease and other predictors of the clinical course after angioplasty.

Patients. Participants were among the previously described 307 consecutive patients who underwent coronary angiography at the University Hospital Maastricht. The inclusion criteria were the same as described in section 2.1.1. Like in section 2.1.3. it was required that 1) patients were symptomatic on admission (angina class II-IV); 2) at least one coronary stenosis was present of $\geq 70 \%$; and 3) PTCA was successful. In view of the hypothesis under investigation it was additionally necessary that the 
patients filled out a valid questionnaire to assess vital exhaustion at two weeks followup and that they were free of recurrent anginal complaints until two weeks after PTCA. Patients who were admitted with acute or recent myocardial infarction were not excluded from the present study to secure sufficient statistical power of the investigation (see below); the possible confounding of this inclusion was statistically controlled for.

Of the 196 patients who underwent PTCA, 127 fulfilled the selection criteria. A detailed description of the prevalence of the inclusion and exclusion criteria will be reported in chapter 5.

Methods. Vital exhaustion was evaluated with the MQ as described in the previous section. It was decided to consider the assessment of exhaustion at two weeks after PTCA only. The rational was that the state of exhaustion before PTCA might be influenced by the severity and duration of anginal complaints. Furthermore, in order to be able to control for the severity of remaining coronary artery disease after successful PTCA, it was conceived that the assessment of vital exhaustion after PTCA would be more valid. At two weeks after angioplasty, restenosis is not yet a probable phenomenon (Liu et al., 1989) and, therefore, the assessment of vital exhaustion at that moment in time was used as the predictor for new cardiac events during followup. As mentioned above, recoil of the dilated lesion in the acute phase after PTCA was controlled for and all successful procedures resulted in a less than $50 \%$ residual stenosis.

New cardiac events were recorded during a follow-up period of 1.5 years. A new cardiac event had to be the consequence of re-emerging coronary ischemia and was defined as one of the following: cardiac death, myocardial infarction, coronary bypass surgery, repeat-PTCA, increase in severity of coronary artery disease, and new anginal complaints with ischemia documented by exercise testing. Information about these events was obtained from the medical records of the University Hospital Maastricht and the regional referring hospitals.

As stated in hypothesis 4 , the severity of coronary artery disease was taken into account in order to control for its predictive value for the clinical course after PTCA. As a measure of the extent of coronary artery disease the same procedure was applied as described in section 2.1.1. This results in a possible range of the extent of coronary artery disease from 1 to 3 vessel disease before PTCA and from 0 to 3 after PTCA. In addition, more subtle ways were applied to assess the severity of coronary artery disease more precisely.

The clinical variables under consideration were: the duration and severity of anginal complaints on admission, the diagnosis upon which PTCA was performed (stable angina, unstable angina, or myocardial infarction), the presence of a positive history of myocardial infarction ( $>1$ month before the current admission) and the left ventricular function. Also the contribution of the standard risk factors for coronary artery disease (smoking, hypercholesterolemia, and hypertension) and demographic characteristics (age, and gender) were considered. The results of a relatively large 
number of studies on the predictors of the clinical course after PTCA do not show a consistent pattern as to the magnitude of the contribution of these clinical and demographic variables. Howewer, on the basis of the validity of these variables in the progression of coronary artery disease in general it was considered to be valid to control for these factors.

Statistical analysis. Univariate association between vital exhaustion, the severity of coronary artery disease, and the other clinical predicator variables and new cardiac events were examined with $\mathrm{Chi}^{2}$ tests. The predictive value of vital exhaustion for new cardiac events during follow-up was evaluated by the Kaplan-Meier curve. The relative contribution of all predictors was assessed by means of multiple logistic regression analyses. Firstly, all sample-specific significant predictors of the clinical course after PTCA were entered in a regression model. Secondly, all theoretically relevant predictors were entered using a stepwise procedure. The details of the procedure and the results are reported in chapter 5 .

\subsection{Blood clotting factors and vital exhaustion.}

One of the mechanisms that may explain the relationship between vital exhaustion and future acute myocardial infarction is an increased tendency to develop blood-clots among exhausted individuals. To circumvent inferential problems related to confounding by manifest coronary artery disease and the use of medication, it was decided to perform a study to investigate this relationship in apparently healthy subjects. A group of vitally exhausted persons was contrasted with a not exhausted control group. These groups were compared on coagulation factors (i.e., substances that promate the formation of a stabilized blood-clot) and fibrinolytic activity (i.e., the substances that indicate the capacity to dissolve a blood-clot that is already present). Normally coagulation and fibrinolysis function in a balanced way. It was hypothesized that in exhausted subjects this balance was disturbed and that coagulation factors were increased and fibrinolytic capacity was reduced. This assumption is summarized in hypothesis 5 .

Hypothesis 5: Vital exhaustion is associated with coagulation and fibrinolytic factors that promote clot-formation and stabilization.

There are numerous factors that promote or inhibit clot-formation and fibrinolysis. Only a limited number of indicators of both systems were investigated in the current study. The rational for choosing these indicators was of two kinds. Firstly, only those factors were investigated that had been found to be associated with both psychosocial stress and future manifestations of acute myocardial infarction. Secondly, the investigation was limited to factors that are of rellevance for the formation and dissolving of 
the stabilized clot, and not to the initial processes of platelet adhesion to the endothelial arterial wall or platelet aggregation (i.e., the formation of the platelet plug). The relationship between vital exhaustion and platelet adhesion and aggregation was investigated by Lulofs (1990) and will be discussed in chapter 7 .

Blood coagulation consists of a complex series of biochemical reactions that result in the loss of fluidity of plasma and the development of a hemostatic clot. This plug becomes stabilized because of the formation of a network of fibrin fibers that captures blood cells and platelets. The present study considers the processes that are involved in the formation and degradation of these fibrin fibers. The essential part of the clotting-process is the formation of thrombin from the $\alpha$-globulin prothrombin which is produced in the liver and present in plasma. Thrombin (factor $\mathbb{I I}_{\mathrm{a}}$ ) proceeds from prothrombin (factor II) as a result of the enzyme prothrombinase (also referred to as thrombokinase or thromboplastin) '. Thrombin has several functions in the clottingprocess: 1) It promotes the transition from fibrinogen (factor I) to fibrin (factor $\mathbb{I}_{2}$ ), which is in fact the ultimate object of the clotting-process; 2) It activates factor XIII which is necessary for the cross-linking of the fibrin fibers in order to stabilize the clot; 3) It stimulates the release reaction of the platelets in which platelet factor 3 , phospholipids, vasoactive amines, and serotonin are secreted; these are factors that promote coagullation and local vasoconstriction; 4) With a positive feedback loop it activates the production of several clotting factors (V, VIII) and also prothrombinase. The present study concerns the intrinsic pathway of the coagulation process because of the absence of tissue damage. The intrinsic pathway is activated when plasma comes into contact with a foreign surface (e.g., a test-tube). This generates 1) an enzyme-like contact-product which activates factor IX (factor IX $\mathrm{IX}_{\mathrm{a}}$ see figure 2.1.), and 2) the release of phospholipids from the platelets. Then, factor $\mathrm{IX}_{\mathrm{a}}$, phospholipids, and in addition, factor $\mathrm{VIII}_{\mathrm{s}}$ and $\mathrm{Ca}^{2+}$ ions ( $=$ factor IV) form an enzyme which activates factor $X$. In addition, factor $X$ is activated by the combined action of tissue factor and factor $\mathrm{VII}_{\mathrm{a}}$. Factor $\mathrm{X}_{\mathrm{a}}$, together with factor $\mathrm{V}_{\mathrm{g}}, \mathrm{Ca}^{2+}$, and phospholipids, promote the transition from prothrombin to thrombin. Finally thrombin (factor $\mathrm{II}_{\mathrm{a}}$ ) splits off two small fibrinopeptides (FPA, and FPB) from the fibrinogen, which will combine to form fibrin. In this clotting-process there are several positive and negative feed-back loops - which are not discussed here - as well as inhibitors. The most prominent of these inhibitors is antithrombin III, which does not only inhibit thrombin (factor $\mathrm{II}_{a}$ ) but also factors $\mathrm{IX}_{\mathrm{a}}, \mathrm{X}_{\mathrm{a}}$, and $\mathrm{XI}_{\mathrm{a}}$. Other inhibitors of coagulation are the vitamin $\mathrm{K}$ dependent protein $\mathrm{C}$, protein $\mathrm{S}$, and also gammaglobulin.

The suffix " $a$ " in, for example, factor $\mathbb{I I}_{\mathrm{a}}$ indicates that the factor has become an active enzyme. 

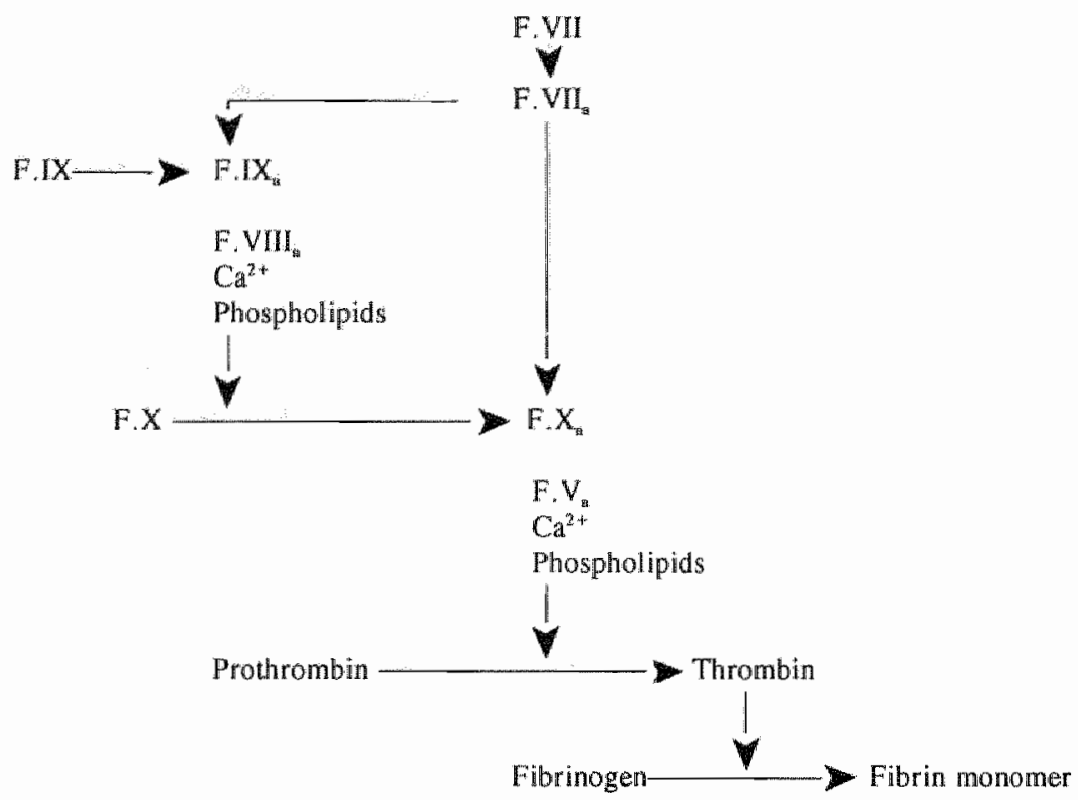

Figure 2.1. Simplified model of the coagulation process that are involved in the formation of a stabilized blood-clot (see text for explanation; feedback mechanisms and inhibitors are not shown).

The other important component of the clotting-process is regulated by the fibrinolytic system. In normal conditions the coagulation system and the fibrinolytic system function in a balanced way, that is, increased coagulation occurs simultaneously with increased fibrinolysis, although most of the times fibrinolysis becomes active after clot-formation has started. The fibrinolytic system is less complex than the coagulation system, but there are activators and inhibitors involved too (see figure 2.2.). The transition from plasminogen into plasmin is crucial for the degradation of the fibrin fibers. Intrinsic activators of fibrinolysis are urokinase plasminogen activator and tissue plasminogen activator (t-PA). t-PA is for the most part contained by the endothelial cells and is released in response to stress, exercise, and injury of the vessell wall. That was the reason to examine t-PA and its inhibitor in the present study. The action of plasmin on the degradation of fibrin is a typical local process and therefore, no plasmin levels can be measured in plasma. It is of interest that the fibrin splitproducts may in combination with thrombin act as an anticoagulant. Important inhibitors of the fibrinolytic process are plasminogen activator inhibitor (PAI) and $\alpha 2$ - 
antiplasmin (serine protease inhibitor - 'serpin'). PAI inhibits t-PA, and therefore higher PAI activity levels indicate a reduced fibrinolytic capacity and thus an increased clotting tendency.

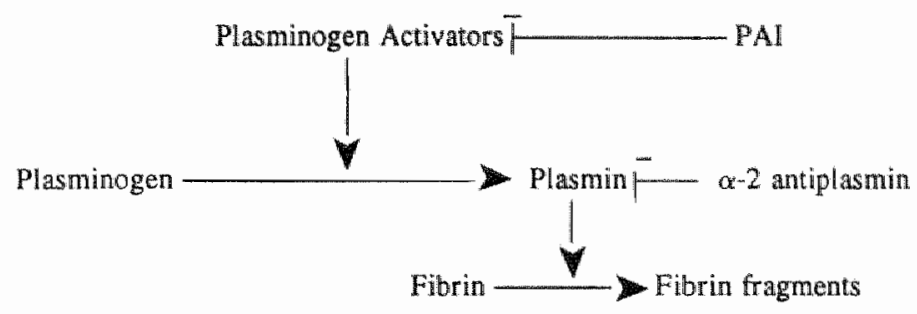

Figure 2.2. The fibrinolytic system consist of both activators and inhibitors of these activators.

Subjects. Participants were recruited from a sample previously described by van Diest (1990). At the time of the present screening these 451 randomly selected men from the city of Maastricht, aged $48-68$ years, were asked to participate in a study on "the relationship between situations of daily life and cardiovascular disease". The invitation included the Maastricht Questionnaire, the Sleep Wake Evaluation List (van Diest, 1990) and a short health status inventory. Seventy-four percent of the 451 men who were invited returned the questionnaires. Exclusion criteria for further laboratory investigations were: coronary artery disease, hypertension, diabetes mellitus, thyroid disease, rheumatoid arthritis, or any other chronic disease, treatment for psychiatric disorder, alcohol or any other substance abuse, current use of medication, and age above 70 or below 35 . These criteria were applied in order to prevent confounding by co-morbidity which is associated with exhaustion (section 1.2.2.) or factors which might confound the measurement of coagulation and fibrinolytic variables (Kluft et al., 1990).

Subjects were classified on the basis of the $M Q$ score as non-exhausted (MQ $\leq 6$ ) or exhausted ( $M Q \geq 16$ ). The prevalence of vital exhaustion in healthy subjects appeared to be unexpectedly low. Only $32(9.6 \%)$ subjects were free of the exclusion criteria and had an MQ score above 16. Not-exhausted subjects were age-matched recruited because age is known to be associated with some coagulation and fibrinolytic factors. Subjects who were eligible for participation on the basis of the inventory were invited to the hospital in order to confirm the absence of the exclusion criteria and to validate the state of exhaustion as assessed with the MQ. Since this procedure revealed seven vitally exhausted subjects, alternative recruitment strategies were applied in order to complete the sample. Two exhausted subjects were recruited from the control group of the study of Meesters (see chapter 1, section 2.2.), one was referred following participation in a research program of the Department of Social Psychiatry 
(by N. Nicolson and M. van Eck), and five exhausted participants of a stress management course were referred by R. Lulofs (Department of Medical Psychology). Four young (age $35-45$ years) not exhausted subjects from the university personal were invited. This was necessary because it appeared that the exhausted subjects who were alternatively recruited were much younger than the average age of the original sample of $451 \mathrm{men}$; and the not exhausted controls had to be recruited on an age-matched basis. This procedure resulted in a final sample of 15 exhausted and 15 not exhausted subjects.

Blood was collected from each subject within two weeks of the interview. A second sample was obtained within three months to control for intra-individual fluctuations.

Methods. Vital exhaustion was initially assessed by means of the mailed Maastricht Questionnaire and, in second instance, validated by the Interview Vital Exhaustion. The interview was described in chapter 1, section 2.2. In brief, a 23-item structured interview is administered of which the content is similar to the Maastricht Questionnaire. For each item with an affirmative response, information about the duration of the symptom is obtained. Exhaustion had to be the consequence of prolonged psychological stress. More specifically, exhaustion was defined as present if 1) the subject had a MQ score $\geq 16$; and 2) there was an increase in one of the components of exhaustion (i.e., lack of energy, irritability, or demoralization) during the last year. This time-related criterion was also applied to exclude subjects with hypochondrial or somatization related complaints. An abbreviated version of the Diagnostic Interview Schedule was administered (Robins et al., 1982) in order to exclude subjects with depressive disorders (chapter 1, section 4.1.). The Symptom Check List (Derogatis, 1986) was used to assess the severity of psychological complaints in comparison to normative data.

As may be evident from the foregoing, a choice had to be made from the numerous factors that are involved in the clotting-process. The rational was to investigate those coagulation factors that were found to be linked to psychosocial factors in previous studies. These were: factor VII, factor VIII, and fibrinogen. As indicators of fibrinolysis t-PA antigen and PAI activity were investigated (due to the procedure of bloodcollection and storage of non-citrated blood, no direct t-PA measurement could be obtained). Details about the techniques of measurement are described in chapter 6 . All coagulation factors are produced in the liver, except factor VIII which is made in the reticulo-endothelial system. Production and degradation of these clotting factors are balanced, which results in relatively stable plasma concentrations of these factors. However upon activation of the clotting-process these concentrations may increase considerably.

Blood chemistry measures were obtained to assess the liver function: bilirubin, the enzymes alanine-amine-transferase (ALAT), aspartate-amine-transferase (ASAT), alkaloid phosphatase, gamma glutamyl-transpeptidase (gamma GT), and lactatedehydrogenase (LDH). For the present study it is of relevance to note that the levels of gamma GT are increased in alcohol abuse. A global indication of the kidney 
function was obtained by measuring the creatinine level. Furthermore, total cholesterol and triglycerides were measured.

Routine hematological measures were obtained immediately after blood-sampling including: hematocrit value, platelet count, and leucocyte count and differential. These measures were collected to rule out confounding by acute phase reaction or by prevalent infections.

Statistical analysis. The average value of the two blood samples were calculated for each variable and each subject separately. The nonparametric Mann-Whitney test was applied on these averaged scores in order to compare the exhausted with the not exhausted group. The results of these analyses will be presented in chapter 6 . 



\section{Chapter 3.}

\section{The relationship between the severity of coronary artery disease and feelings of exhaustion'}

As discussed in chapter 1 , excessive fatigue and feelings of general malaise are anong the most prevalent premonitory symptoms of myocardial infarction and sudden cardiac death (e.g., Kuller et al., 1972; Romo, 1973; Feinleib et all., 1975; Fraser, 1978; Klaboe et al., 1987). Psychological analysis of the tiredness prior to myocardial infarction revealed that it typically consists of three components: lack of energy, increased irritability, and demoralization. We have labeled this state previously as 'vital exhaustion' (Appels, 1992). A case-control study (Falger \& Schouten, 1992) and two prospective studies (Appels et al., 1987; Appels \& Otten, 1992) showed that feelings of exhaustion were predictive of first myocardial infarction in apparently healthy individuals. Moreover, this association was independent of the known risk factors for coronary artery disease.

The origin of vital exhaustion as a premonitory symptom of clinical manifestations of coronary artery disease is still not well understood. In the late fifties, Selye (1977) postulated that a state of exhaustion reflects a breakdown in adaptation to prolonged psychological stress. Congruent with this notion is the observation by Falger and Schouten (1992), in which a number of stressful life-events preceded exhaustion in patients who suffered myocardial infarction (see also chapter 1, section 3).

Alternatively, it may be that exhaustion prior to myocardial infarction is caused by underlying coronary disease or a reduced pump-function of the heart. In the prospective studies of Appels and colleagues (1987; 1992), subjects were excluded if they had anginal complaints or signs of past myocardial infarction according to the 12 -lead electrocardiogram at rest. However, this procedure is obviously not adequate to rule out the presence or absence of coronary disease. The observed relationship between exhaustion and myocardial infarction might have been confounded by undetected underlying coronary artery disease. That is, subjects where coronary arteries were most affected by atherosclerotic narrowing, and who were for that reason at the highest risk of developing climical endpoints, may also have reported exhaustion more often.

The purpose of the present study was to investigate the relationship between the extent of coronary artery disease and cardiac pump function on the one hand, and vital exhaustion on the other hand. To this end, a group of patients who underwent

This chapter is submitted by: Kop WJ, Appels A, Mendes de Leon CF, Bär FW. 
diagnostic coronary angiography was evaluated for feelings of exhaustion. In order to examine the possibility that feelings of exhaustion prior to myocardial infarction reflect underlying coronary disease, the following hypotheses were tested: Hypothesis 1. Vital exhaustion is related to the extent of coronary artery disease. Hypothesis 2: Vital exhaustion is related to a poor pump function of the heart in patients with coronary artery disease. If exhaustion and coronary artery disease are found to be strongly related, then there would be little need to search for alternative explanations for the tiredness before myocardial infarction.

\subsection{Methods.}

\subsubsection{Patients.}

The study population consisted of 322 consecutive patients referred for diagnostic coronary angiography. Patients had to give informed consent and the following inclusion criteria were applied: 1) age $\leq 70$ years; 2 ) no previous revascularization by coronary angioplasty or bypass surgery; and 3) absence of severe co-morbidity (e.g., valvular disease, heat failure, cancer, or psychiatric disorder). Fifteen patients refused to participate or returned an invalid questionnaire for the assessment of exhaustion, leaving 307 patients for further analysis (response rate $95 \%$ ).

\subsubsection{Vital exhaustion.}

The Maastricht Questionnaire (MQ) was used to assess feelings of exhaustion. The MQ consists of 21 items and has a high internal consistency (Crombach's $\alpha=0.89$ ). In a healthy population the mean MQ score is 8.8 (s.d. $=8.7$, median $=6$, possible score range: $0-42$ ). In this study, patients were asked to report about how they felt during the three months preceding the current admission.

\subsubsection{Coronary artery disease and left ventricular function.}

Coronary angiograms were evaluated by a panel of experienced cardiologists. Two indices of coronary disease severity were used. The first was the number of vessels with a lesion $>50 \%$ (i.e., right coronary artery, left anterior descending artery, and circumflex artery). The possible range of this variable (VD) is $0-3$. The other, more informative, measure of coronary artery disease takes both the severity of the coronary lesions as well as the number of diseased vessels into account (Williams et al., 1988), making use of the following criteria: $0=$ no coronary artery disease; $1=$ clinically minor disease in one ore more coronary arteries (i.e., lesion $\leq 70 \%$ ); $2=$ $>70 \%$ stenosis in one coronary artery; $3=>70 \%$ stenosis in two coronary arteries; $4=>70 \%$ stenosis in three coronary arteries; $5=>70 \%$ stenosis in the left main artery. This variable is referred to as CADSEV. 
The left ventricular function was evaluated using the standard procedure of ventricular angiography. The left ventricular ejection fraction was calculated by dividing the stroke volume (i.e., the difference between the end-diastolic and end-systolic volume of the left ventricle) by the end-diastolic volume. In healthy persons the left ventricular ejection fraction is $67 \pm 8 \%$. In the present study a distinction was made between patients with a 'poor' $(\leq 40 \%)$, "moderate' $(40-60 \%)$, and 'good' $(>60 \%)$ left ventricular ejection fraction.

\subsubsection{Clinical and demographic characteristics.}

Information about the patient characteristics was obtained from the medical record. The duration of cardiac complaints and the exercise performance were investigated to assess the impact of the patient's clinical condition prior to admission on exhaustion. Maximal exercise performance was evaluated using the Bruce protocol (Bruce, 1971) if the test was done within the last three months before admission. Exercise performance was defined as "poor' if the patients reached stage $\mathbb{I}$ or $\mathbb{I}$, and as 'good" if stage III, IV, or $\mathrm{V}$ was reached. The presence of peripheral vascular disease, a positive history of myocardial infarction (i.e., occurring more than one month before hospitalization), hypertension, and diabetes mellitus were also considered as relevant indicators of the clinical condition prior to hospitalization.

The diagnosis on admission could be either stable angina, unstable angina, or myocardial infarction. Unstable angina was defined as angina at rest, progressive angina, or resent onset angina. myocardial infarction was diagnosed on the basis of the electrocardiogram and subsequent enzyme rise. The New York Heart Association classification criteria for angina and dyspnea were used to assess the severity of cardiac complaints.

Because fatigue can be a possible side-effect of several types of medications that are frequently prescribed to coronary patients, we registered the use of: nitrates, Bblocking agents, calcium antagonists, platelet aggregation inhibitors, coumarins, and diuretics.

Demographic variables included: age, gender, education, and marital status. Education was divided into three categories: 'low' (less than 8 years), 'middle' ( 8 to 12 years), and 'high" (more than 12 years). Marital status was categorized as: married or living with a spouse, single, divorced, and widowed.

\subsubsection{Statisticall analysis.}

Analyses of variance were used to examine the association between the extent of coronary disease, the clinical, and demographic variables on the one hand, and vital exhaustion on the other. Next, the multiple correlation coefficient $\left(R^{2}\right)$ was used as a measure of the explained variance of the $\mathrm{MQ}$ scores. $\mathrm{R}^{2}$ is also referred to as the 'coefficient of determination' because it provides an indication as to what extent a set 
of predictor variables accounts for the variation of a dependent variable (i.c., the exhaustion score). Because $\mathrm{R}^{2}$ tends to increase when the number of predictor variables becomes larger, the adjusted $R^{2}$ was used as a measure of association.

\subsection{Results.}

The mean age of the study population was $55.9 \pm 8.5$ years and 63 patients $(21 \%)$ were women. The relationship between extent of coronary disease and exhaustion is shown in Table 1. The number of diseased vessels (VD) was not associated with the mean MQ score, nor was there a relationship between the more informative variable CADSEV and exhaustion. Because the association between VD and exhaustion was stronger than the association between CADSEV and exhaustion, the former was used in the multiple regression analyses as an indicator of the extent of coronary artery disease.

Table 3.1. CORONARY ARTERY DISEASE AND LEFT VENTRICULAR FUNCTION AS RELATED TO VITAL EXHAUSTION.

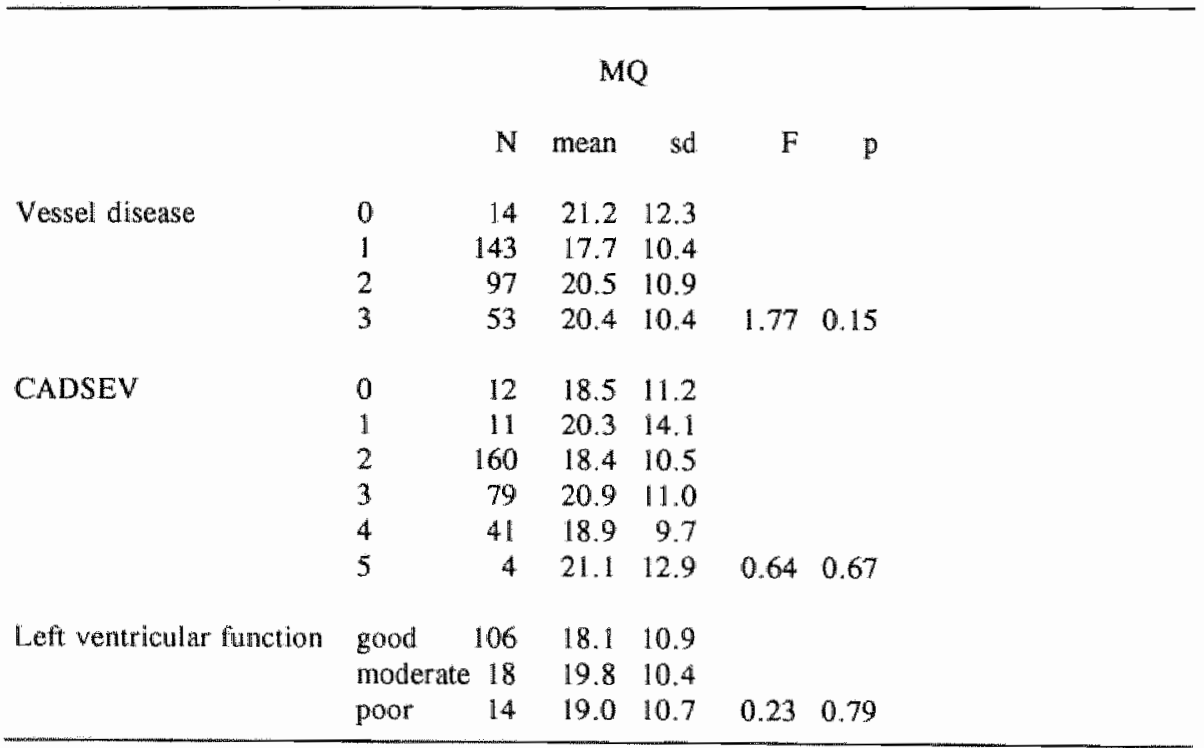

CADSEV = a measure of the severity of coronary disease, see text for explanation.

Left ventricular ejection fraction was obtained in 138 patients $(45 \%)$. Table 1 also shows that a poor left ventricular function was not very prevalent in the present sample $(\mathbf{N}=14)$. In addition, patients with a poor or moderate function of the left ventricle did not have higher $\mathrm{MQ}$ scores than patients with a good left ventricular function. 
As for the relationship between the clinical condition prior to admission and exhaustion, Table 2 shows that a longer duration of cardiac complaints and poor exercise performance were associated with higher exhaustion scores. The presence of peripheral. vascular disease was also related to more exhaustion. Hypertension and diabetes mellitus did not influence exhaustion scores, but in the case of diabetes the non-significant effect may have been caused by a lack of statistical power. With regard to the diagnosis and severity of cardiac complaints on admission, it was found that patients with unstable angina tended to have higher MQ scores than patients with myocardial infarction or stable angina, but the severity of anginal complaints was not related to the MQ. Furthermore, patients who had dyspnea (class II, III, or IV) had higher MQ scores than patients without dyspnea.

Elevated MQ scores were observed when patients used one of the following medications: nitrates, B-blocking agents, calcium antagonists, or diuretics. Sixty patients were not on any medication on admission, this group had lower exhaustion scores than patients who used one or more medications (mean MQ scores $14.4 \pm 9.1$ and $20.4 \pm 10.7$, respectively; $F=15.8 ; p<0.001$ ).

The demographic characteristics were positively related to exhaustion in the sense that women scored higher on the MQ than men did (mean MQs $23.4 \pm 10.3$ and 18.1 $\pm 10.5 ; \mathrm{F}=12.8 ; \mathrm{p}<0.001)$. Patients with a low level of education had higher MQ scores compared to those with a middle or high level of education (mean MQ scores: $22.5 \pm 11.4,19.6 \pm 10.0$, and $15.6 \pm 10.2$, respectively; $F=10.3 ; p<$ $0.001)$. Age was not correlated with exhaustion scores $(r=0.01)$ and there was no relationship between marital status and exhaustion.

Multiple regression analyses were carried out in order to estimate the contribution of the extent of coronary disease, the clinical characteristics, medication, and the demographic variables in the prediction of exhaustion scores. In the first regression analysis, VD was entered as predictor variable of the MQ score. It appeared that VD explained less than $1 \%$ of the variance of exhaustion scores $\left(R^{2}=0.003 ; \mathbb{F}=2.1 ; p\right.$ $=0.15$ ). The clinical characteristics (i.e., duration of complaints, exercise performance, history of myocardial infarction, hypertension, peripheral vascular disease, diagnosis on admission, severity of angina, and severity of dyspnea) explained $4 \%$ of the variance of exhaustion $\left(\mathrm{R}^{2}=0.04 ; \mathrm{F}=11.9 ; \mathrm{p}=0.07\right)$. In this model, peripheral disease $(p=0.03)$ and dyspnea $(p=0.09)$ were the clinical variables that contributed most to the prediction of the MQ score. When the type of medication was regressed on the MQ score, the explained variance was $4 \%\left(R^{2}=0.04 ; F=3.3 ; p=0.003\right)$, which was due to the contribution of B-blocking agents $(p=0.07)$, calcium antagonists $(p=0.08)$, and diuretics $(p=0.02)$. Next, the demographic characteristics were regressed on the $\mathrm{MQ}$ scores, which revealed an explained variance of $9 \%\left(\mathrm{R}^{2}=0.09\right.$; $\mathbb{F}=8.1 ; p<0.0001)$. Gender $(p=0.005)$, marital status $(0.04)$, and education ( $p$ $=0.0003$ ) contributed to the prediction of the MQ score. Finally, the extent of coronary disease, the clinical characteristics, the number of medications used, and the demographic variables were entered simultaneously into one model. This analysis 
chaprer 3

revealed that the overall explained wariance was $8 \%\left(\mathrm{R}^{2}=0.08 ; \mathrm{F}=2.1 ; \mathrm{p}=0.01\right)$, in which education was the only variable that substantially contributed to the prediction of the MQ score $(\mathrm{p}=0.02)$.

Table 3.2. THE RELATIONSHIP BETWEEN CLINICAL CHARACTERISTICS AND EXHAUSTION.

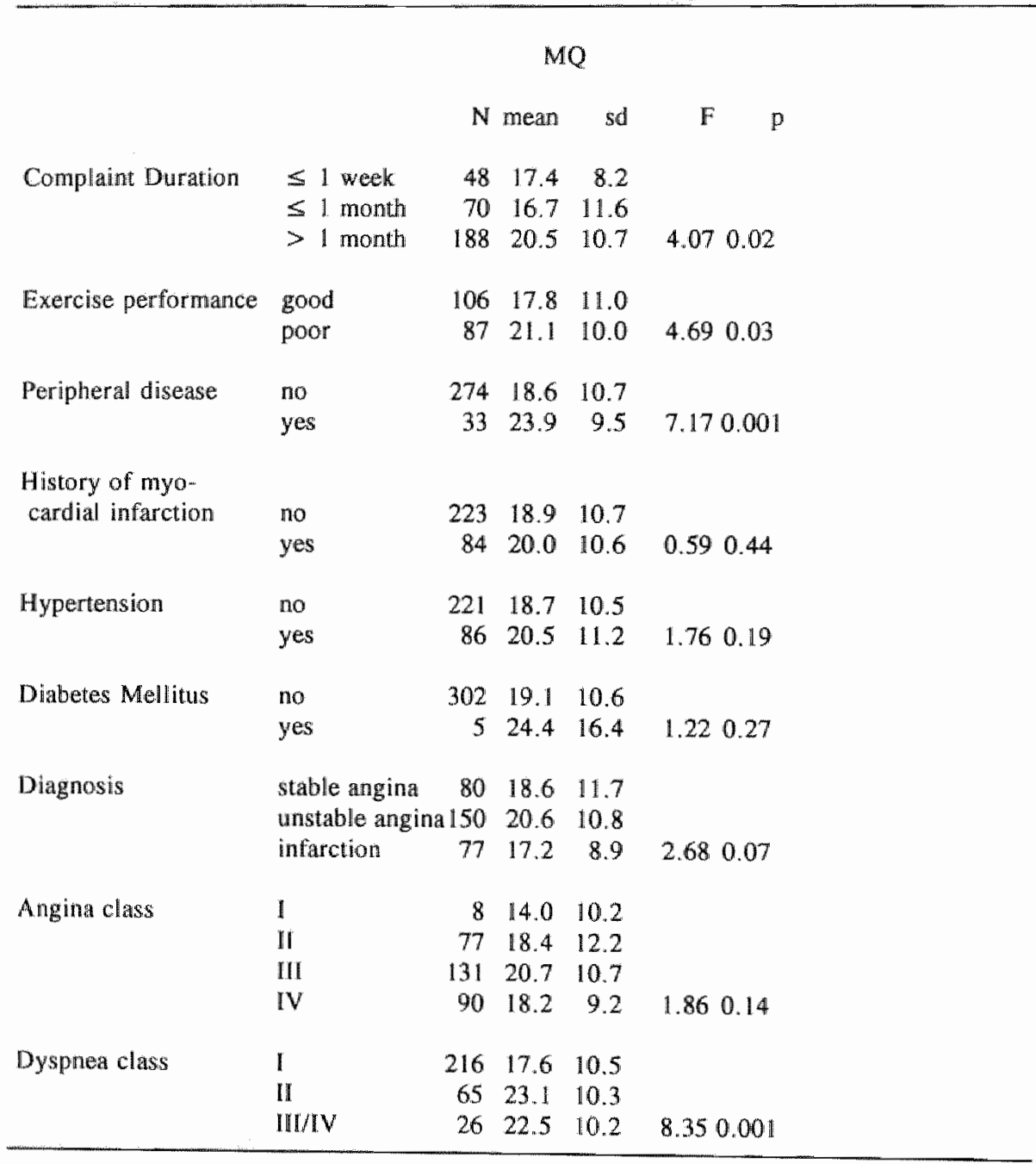




\subsection{Discussion.}

Although feelings of unusual tiredness and exhaustion are among the most prevalent premonitory symptoms of myocardial infarction and sudden cardiac death, the exact origin of these feelings is not well understood. The current study shows that there is no relationship between the severity of coronary disease and exhaustion. Less than $1 \%$ of the variance in exhaustion scores could be explained by the severity of coronary artery disease. Clinical characteristics explained $4 \%$ of the variance of the exhaustion scores, while the demographics and use of medication accounted for $9 \%$ and $4 \%$, respectively. Multiple regression analysis revealed that the demographic variables were the best predictors of vital exhaustion.

The cross-sectional design of the present study causes some inferential problems. One possible confound is related to patient-based and physician-based referral-bias. It is obvious that patients who have coronary disease were over-represented in the present sample in comparison with the prevalence in the general population. Furthermore, it is possible that cardiologists consider complaints of undue tiredness as angina equivalents and hence refer individuals with these symptoms more often for coronary angiography. In other words, there is an over-selection of the disease and of the exposure. This may cause both over-estimation as well as under-estimation of the true relationship between severity of coronary disease and exhaustion, depending on the configuration of the selection fractions (Ragland et al., 1991). The deviant psychological characteristics of patients who do not show signs of coronary artery disease at angiography (e.g., Pickering et al., 1985) may have influenced the results as well. However, these factors are not likely to fully account for the very low magnitude of the association between feelings of exhaustion and severity of coronary artery disease.

There may be several other plausible explanations for the occurrence of vital exhaustion in patients referred for coronary angiography. Impaired left ventricular function may be one of the possible causes of exhaustion. Although no association was found between a reduced left ventricular function and exhaustion scores, it should be noted that the pump function was assessed making use of the ventricular angiogram at rest. Thus, this study can not exclude the possibility that insufficient increase of the cardiac pump function during exercise is associated with feelings of exhaustion. The present study does not allow to address this issue. We also examined what other clinical characteristics influenced vital exhaustion. It was shown that a longer duration of complaints, reduced exercise performance, peripheral vascular disease, and dyspnea were related to feelings exhaustion. This means that these factors probably promoted extreme tiredness. It may be that an additional process occurs as well, in the sense that the physical condition and the psychological reaction to it are mutually reinforcing phenomena. For example, reduced exercise performance is likely to induce feelings of tiredness and exhaustion. This may bring about that a person refrains from physical activity, which in turn decreases exercise performance. In this way a gradual but persistent worsening of the physical and psychological well being devellops. Support 
chapter 3

for this argument is found in the present observation that exhaustion is related to the duration of complaints and not to the severity of underlying coronary artery disease. However, only $4 \%$ of the exhaustion scores was accounted for by the clinical characteristics.

In conclusion, previous prospective studies showed that feelings of exhaustion are of predictive value for future myocardial infarction (Appels et al., 1987; 1988; 1989; 1992). Until now, it could not be excluded that this association was confounded by subclinical underlying coronary artery disease. From the present study it can be inferred that feelings of exhaustion are not likely to be result of impaired coronary perfusion due to coronary artery disease. Therefore, feelings of exhaustion have to be distinguished from the typical picture of tiredness on exertion in coronary patients which is caused by insufficient coronary supply. The present observations are in support of the notion that vital exhaustion may reflect an end-stage of prolonged psychological stress. 


\section{Chapter 4.}

\section{The effect of successful coronary angioplasty on feelings of exhaustion."}

The tiredness that is often seen as a premonitory symptom of myocardial infarction is a rather aspecific complaint and difficult to use in the diagnostic process. In the previous chapter it was shown that there was not a clear association between the severity of coronary artery disease and vital exhaustion. As discussed more extensively in chapter $I$, the typical clinical picture of feelings of exhaustion before myocardial infarction consists of three elements: lack of energy, increased irritability and demoralization (Appels, 1990). Feelings of exhaustion were found to be a precursor of first myocardial infarction in a 4-year follow-up study in 3877 healthy males (Appels et al., 1987; 1988; 1989) with a relative risk of 2.28 . The estimated relative risks for the four succeeding years of follow-up were $10.05,2.03,3.04$ and 0.68 , respectively, which indicates that exhaustion is particularly a short-term precursor of myocardial infarction. Although the prospective design of that study ruled out that the observed association between exhaustion and future fatal or non-fatal myocardial infarction was caused by manifest coronary artery disease, subclinical coronary disease could explain the elevated risk of myocardial infarction.

To shed more light on this issue, the present study investigates the relationship between the severity of coronary artery disease and exhaustion and the effect of successful coronary revascularization by Percutaneous Transluminal Coronary Angioplasty (PTCA) on feelings of exhaustion. We tested if hypothesis 3." Restoration of coronary perfusion by successful coronary angioplasty is followed by a substantial reduction of vital exhaustion could be confirmed. The following questions are addressed: 1) What is the magnitude of the association between feelings of exhaustion and the extent of cononary artery disease before and after PTCA? 2) Does successful restoration of coronary perfusion through PTCA result in a marked improvement of exhaustion? 3) What is the long term effect of PTCA on feelings of exhaustion? We assume that feelings of exhaustion primarily reflect prolonged psychological tension, rather than (sub)clinical coronary disease. However, if the present study reveals that exhaustion is strongly associated with atherosclerosis and markedly improves following restoration of coronary blood-flow, there would be no need for further studies that investigate the psychological origin of exhaustion in patients at risk of myocardial infarction.

This chapter is in press: W.J. Kop, A.P.W.M. Appels, C.F. Mendes de Leon, H. de Swart, and F.W. Bär. The effect of successful coronary angioplasty on feelings of exhaustion. Int $J$ Cardiol. 
chapter 4

\subsection{Methods.}

\subsubsection{Patients.}

Subjects consisted of consecutive patients referred for elective coronary angioplasty. The study was approved by the human research committee of the University Hospital of Maastricht. All patients had to be symptomatic (class II-IV according to the New York Heart Association classification) and had to give informed consent. In addition, it was required that an angiographically documented stenosis of $\geq 70 \%$ was present in at least one coronary artery. Patients were excluded if they were older than 70 years, had recent myocardial infarction (i.e. $<1$ month before admission), previous PTCA or coronary artery bypass grafting, valvular disease, other disabling cardiac conditions, or severe comorbidity.

Since the aim of the present study was to investigate whether restoration of coronary blood supply results in an amelioration of exhaustion, only patients with a successful PTCA were included. A successful PTCA was defined as a residual stenosis of the culprit lesion of less than $50 \%$. In case of multiple lesions (e.g. in multi-vessel disease), it was required that at least one lesion was dilated successfully.

In total., 157 patients were eligible for the study. PTCA was considered to be fully successful in 129 patients (success-rate $82 \%$ ). Nine patients were excluded because of incomplete data on exhaustion on admission, leaving 120 patients for further analyses.

\subsubsection{Procedure.}

Exhaustion was assessed by means of the Maastricht Questionnaire (MQ; Appels et al., 1987). The $\mathrm{MQ}$ consists of 21 items and inquires about fatigue, sleep problems, demoralization, and irritability. The range of scores is from 0 to 42 , higher scores indicating more severe exhaustion. In a healthy population the mean score of the $\mathrm{MQ}$ is 8.8 (standard deviation $=8.7$, median $=6$ ). In hospitalized patients with other than cardiac diseases the MQ tends to be moderately elevated (mean $=11.7$, standard deviation $=9.8$; Falger, 1989; see also chapter 1, section 2.2.).

The first assessment of exhaustion was done while the patient was hospitalized for PTCA. Patients received the questionnaire before the actual procedure and were asked to give information about feelings of exhaustion during the preceding three months. 71 (59\%) actually completed the MQ before the actual procedure, while the other patients completed the MQ in the hospital but after the PTCA. This did not result in differences between MQ scores on admission or at follow-up and therefore both groups will be combined in the further analyses. Follow-up assessment of exhaustion was obtained at two weeks and six months after PTCA. The time of evaluation at two weeks was chosen to reveal the short-term effect of PTCA on exhaustion, assuming that restenosis is not yet present at that time (e.g., Liu et al., 1989). Follow-up assessment of exhaustion was complete in 108 patients at two weeks $(90 \%)$ and in $85(75 \%)$ at six 
months. Questionnaires were not used if they were returned more than two weeks too late. Patients with missing $\mathrm{MQ}$ data at follow-up did not differ from those who returned valid follow-up questionnaires on any of the independent variables, nor on the initial MQ score.

\subsubsection{Severity of coronary artery disease and clinical control variables.}

The extent of coronary disease was measured as the number of diseased vessels and also as the number of lesions $>50 \%$. During PTCA, 147 of the 202 lesions were approached, of which 139 were dilated successfully $(95 \%)$. If there were multiple lesions, the number of arteries with non-dilated significant lesions $(>50 \%)$ was registered. This number was addied to the number of arteries with an unsuccessfully dilated lesion and the sum is referred to as the "extent of coronary disease after PTCA'. This variable is used as a measure of restoration of coronary perfusion by PTCA. Of the 42 patients who had multi-vessel disease, 29 were dilated in one artery only. This resulted in 78 patients $(65 \%)$ who had no significant lesions after PTCA, 32 patients $(27 \%)$ who continued to have a significant lesion in one coronary artery and 10 patients $(8 \%)$ with a lesion in more than one coronary artery.

Left ventricular function was evaluated by means of angiography or echocardiography. If the ejection fraction was less than $40 \%$, the left ventricular function was considered as poor.

Information about the clinical characteristics was obtained from the medical records and included: exercise tolerance (following the Bruce protocol), duration of symptoms before admission, severity of angina (according to the New York Heart Association classification), the presence of dyspnea on admission, and history of myocardial infarction. Demographic control variables included: age, sex and education. Education was divided into three categories: low (less than 8 years), middle (8 to 12 years) and high (more than 12 years). Since fatigue has been described as a possible side-effect of several types of medications, the use of the following was registered: nitrates, $B$ blocking agents, calcium antagonists, platelet aggregation inhibitors, coumarins, and diuretics.

\subsubsection{Statistical analysis.}

The association between extent of coronary disease and other patient characteristics with exhaustion before and after PTCA was examined by univariate F-tests. The tertiles of the $\mathrm{MQ}$ in the healthy population were used as reference for dividing patients into three groups: 'not exhausted' (MQ < 4), 'moderately exhausted' $(\mathrm{MQ}=$ 4-11) and 'exhausted' (MQ $\geq 11)$. Multiple regression analyses were applied to investigate how much of the variance of the MQ scores could be explained by the extent of coronary disease and the clinical characteristics (indicated by $R^{2}$ ). $R^{2}$ expresses to what degree values of the dependent variable (i.e., the MQ) are deter- 
mined by a set of independent variables (i.e., the extent of coronary disease and control variables).

The short-term effect of PTCA on exhaustion was evaluated by paired t-test. The importance of the presence of nondilated lesions after PTCA as related to the MQ at two weeks follow-up was examined by analysis of variance. In order to examine the predictors of the change in exhaustion following PTCA, a difference score was calculated (subtracting the MQ at two weeks after PTCA from the MQ before PTCA). This difference score was entered as dependent variable in a regression model, with extent of coronary disease after PTCA, clinical characteristics, and MQ score on admission as predictor variables.

The long-term effect of PTCA on exhaustion was investigated at six months after the procedure. Patients who remained free of typical angina during follow-up were compared with patients who had typical anginal complaints. For this purpose a $2 \times 3$ analysis of variance was used with the two groups (with, and without angina) as between-subjects factor, and the MQ scores as a three-level within-subject factor. Patients who had a second revascularization or myocardial infarction during follow-up were excluded from this analysis, because this might confound the assessment of exhaustion. All analyses were performed with the SPSS-PC software package (Norusis, 1986).

\subsection{Results.}

\subsubsection{Exhaustion before PTCA.}

The mean age was $56.0 \pm 9.2$ years (range 33 to 70 ). Twenty-six patients (22\%) were women. The mean MQ score before PTCA was $20.0 \pm 10.9$, which indicates that patients with PTCA had on the average higher MQ scores than what we have previouslly found in the general population and in patients with non-cardiac diseases. When compared with the tertile distribution of the MQ in the general population, $75 \%$ were 'exhausted" "18\% "moderately exhausted", and 7\% 'not-exhausted' before PTCA.

The univariate association between MQ scores and clinical characteristics are shown in Table 1. The extent of coronary artery disease was related to higher MQ scores (F $=4.7$, d.f. $117,2, p=0.01)$. The number of patients with three-vessel disease was small $(\mathbb{N}=6)$, but their $M Q$ scores were similar as in patients with single-vessel disease. The total number of lesions before PTCA was also significantly related to the MQ scote $(F=4.3$, d.f. $117,2, \mathrm{E}=0.02)$. There was no significant relationship between a poor left ventricular function and vital exhaustion. 
Table 4.1. VITAL EXHAUSTION AS RELATED TO CLINICAL CHARACTERISTICS IN PATIENTS REFERRED FOR PTCA

\begin{tabular}{|c|c|c|c|c|c|}
\hline & & $\bar{N}$ & meant & s. & $\mathrm{p}$ \\
\hline \multirow[t]{3}{*}{ Vessels diseased } & 1 & 78 & 18.0 & 10.9 & \\
\hline & 2 & 36 & 24.5 & 9.9 & \\
\hline & 3 & 6 & 19.3 & 10.6 & 0.01 \\
\hline \multirow[t]{3}{*}{ Number of lesions } & 1 & 66 & 17.5 & 11.2 & \\
\hline & 2 & 36 & 23.6 & 9.4 & \\
\hline & $\geq 2$ & 18 & 22.2 & 10.9 & 0.02 \\
\hline \multirow[t]{2}{*}{ LV fumction } & poor & 4 & 13.6 & 129 & \\
\hline & good. & 96 & 20.8 & 10.9 & nis: \\
\hline \multirow[t]{3}{*}{ Exercise toleranco } & Bruce I, or II & 32 & 22.3 & 9.7 & \\
\hline & Bruce III, or W & 40 & 18.4 & 12.4 & \\
\hline & not made & 48 & 19.8 & 10.3 & ns \\
\hline \multirow{2}{*}{ Duration of complaints } & $\leq \mathbb{1}$ month & 31 & 15.7 & 10.9 & \\
\hline & $>1$ month & 88 & 21.4 & 10.6 & 0,01 \\
\hline \multirow[t]{3}{*}{ Angina (class) } & II & 38 & 18.0 & 12.0 & \\
\hline & MII & 62 & 21.6 & 10.5 & \\
\hline & IV & 20 & 19.0 & 9.9 & nis: \\
\hline \multirow[t]{2}{*}{ Dyspnea (class) } & 1 & 78 & 18.9 & 10.9 & \\
\hline & II-IW & 42 & 22.1 & 10.7 & mas \\
\hline \multirow[t]{2}{*}{ History of MI } & no & 92 & 20.1 & 11.2 & \\
\hline & yes & 28 & 19.9 & 10.3 & ns \\
\hline \multirow[t]{2}{*}{ Age } & $\leq 55$ & 58 & 22.2 & 11.3 & \\
\hline & $>55$ & 62 & 18.0 & 10.2 & 0.04 \\
\hline \multirow[t]{2}{*}{ Gender } & matle & 94 & 18.9 & 11.0 & \\
\hline & female & 26 & 24.0 & 9.7 & 0.04 \\
\hline \multirow[t]{3}{*}{ Education } & low & 37 & 24,0 & 10.6 & \\
\hline & middle & 40 & 19.4 & 10.1 & \\
\hline & thigh & 39 & 16.2 & 11.1 & 0,008 \\
\hline \multirow[t]{2}{*}{ Nitrates } & no & 31 & 17.4 & 11.4 & \\
\hline & yes & 89 & 20.9 & 10.7 & ns \\
\hline \multirow[t]{2}{*}{ B-blocking agents } & not & 46 & 18.8 & 11.4 & \\
\hline & yes & 74 & 20.7 & 10.6 & Dis \\
\hline \multirow[t]{2}{*}{ Calcium antagonisits } & no & 51 & 19.9 & 10.8 & \\
\hline & yes & 69 & 20.1 & 11.1 & thes \\
\hline \multirow{2}{*}{$\begin{array}{l}\text { Platelett aggregation } \\
\text { inthibitors. }\end{array}$} & no & 37 & 20.8 & 11.8 & \\
\hline & yes & 83 & 19.6 & 10.6 & this \\
\hline \multirow[t]{2}{*}{ Coumarins } & no & 114 & 19.9 & 11.1 & \\
\hline & $y e s$ & 6 & 23.0 & 80 & nuts \\
\hline \multirow[t]{2}{*}{ Diuretices } & no & 108 & 19.8 & 11.0 & \\
\hline & $y$ & 12 & 21.6 & 10.4 & ass \\
\hline
\end{tabular}

$L V=$ Left ventricular; $M I=$ Myocardial infarction; $n s=p>0.1$. 
The relationship between $\mathrm{MQ}$ and the clinical characteristics are also shown in Table 1. If anginal complaints lasted longer than one month the MQ score was higher than in case of shorter duration of symptoms $(F=6.7, p=0.01)$. However. exhaustion was not related to the sewerity of angina, nor to the maximal exercise tolerance. Type of medication was also not significantly related to higher $\mathrm{MQ}$ scores $(\mathrm{p}>0.1)$. Differences between patients who were on one of these medications compared to those who were not may have been obscured by the overall high exhaustion scores and the low prevalence of patients who were free of any kind of medication in the present sample.

Women had higher MQ scores than men (mean MQ scores 24.0 and 18.9 respectively; $F=4,5 \quad p=0.04$ ). Although there was no correlation between age and $\mathrm{MQ}$ score $(r=-0.06)$, patients younger than 56 years had higher $M Q$ scores $(F=4.6, p$ $=0.04)$. A lower educational level was related to higher $\mathrm{MQ}$ scores $(\mathrm{F}=5.1, \mathrm{p}=$ 0.008 ).

Multiple regression analysis showed that $4 \%$ of the variance in the exhaustion scores was determined by the extent of coronary artery disease $\left(R^{2}=0.04, F=5.1\right.$, $\mathrm{p}=0.03)$. Addition of the clinical characteristics resulted in an increase of the explained variance by $8 \%\left(\mathrm{R}^{2}=0.12, \mathrm{~F}=2.6, \mathrm{p}=0.03\right)$. Addition of the demographics increased the explained variance to $21 \%$. Table 2 shows the results of the Jatter multiplle regression analysis; the number of diseased vessels and the duration of complaints are positively associated with the MQ scores, while the educational level contributed in an opposite direction $\left(\mathbb{R}^{2}=0.21, F=3.1, p=0.002\right)$.

Table 4.2. THE PREDICTION OF VITAL EXHAUSTION BEFORE PTCA FROM EXTENT OF CORONARY DISEASE, CLINICAL AND DEMOGRAPHIC PATIENT CHARACTERISTICS

\begin{tabular}{lrrr}
\hline & $B$ & $t$ & $p$ \\
Education & -0.27 & -2.9 & 0.005 \\
Angina class & 0.12 & 1.3 & 0.212 \\
History of MI & -0.09 & -1.0 & 0.305 \\
Age & -0.15 & -1.7 & 0.091 \\
Exercise tolerance & -0.05 & -0.6 & 0.546 \\
Number of vessels diseased & 0.23 & 2.6 & 0.011 \\
Dyspnea & 0.05 & 0.5 & 0.613 \\
Duration of complaints & 0.19 & 2.0 & 0.047 \\
Gender & 0.04 & 0.4 & 0.684 \\
\hline
\end{tabular}

$B=$ standardized regression coefficient in the multiple regression model.

MI = Myocardial infarction. 


\subsubsection{The effect of PTCA on exhaustion.}

The mean MQ scores decreased from $20.2 \pm 11.0$ before PTCA to $16.5 \pm 11.3$ at

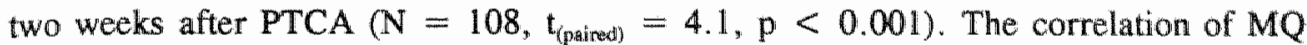
scores before PTCA and at two weeks follow-up was $0.65(\mathrm{p}<0.001)$. A substantial number of patients continued to be exhausted; the percentage 'exhausted' patients decreased from $75 \%$ to $65 \%$, while $19 \%$ were "moderately exhausted" two weeks after PTCA, and 16\% were 'not-exhausted' (Figure 1).

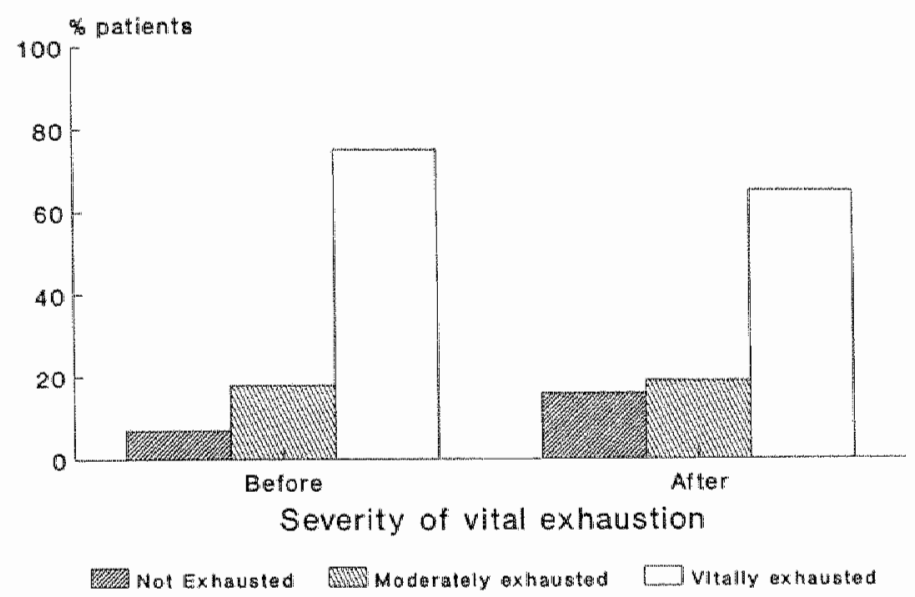

Figure $\mathbb{1}$. Exhaustion before and two weeks after successful PTCA. Patients are allocated to three categories: 'not exhausted', "moderately exhausted" and "exhausted' in accordance with the distribution of the $\mathrm{MQ}$ in the general population.

It was found that the extent of coronary disease after PTCA was not significantly associated with higher MQ scores two weeks after PTCA ( $F=1.9$, d.f. $=105,2, \mathrm{p}$ $=0.16$; Figure 2).

Next, the change in MQ scores was related to the extent of coronary disease after PTCA, the clinical and demographic characteristics, as well as the MQ score on admission. The explained variance was $21 \%(\mathrm{~F}=2.2, p=0.02)$. The MQ on admission was the only significant predictor of the MQ difference score $(p<0.001)$ and none of the clinical variables contributed significantly to the prediction of the change in MQ score.

To evaluate the state of exhaustion six months after discharge, a distinction was made between patients free of typical anginal complaints at the end of follow-up $(\mathrm{N}=53)$, and those suffering typical angina $(\mathrm{N}=19)$. Analysis of variance revealed 
that the MQ at two weeks and six months was significantly lower than before PTCA $(\mathrm{F}=7.1, \mathrm{~d} . \mathrm{f} .=140,2, \mathrm{p}=0.001)$. But there appeared to be no differences between those with and without angina $(F=2.3$, d.f. $=70,1, p=0.13)$. The interaction between the change in the three MQ assessments and the presence or absence of angina was also not significant $(F<1)$. Sixty percent of the patients were exhausted at six months follow-up.

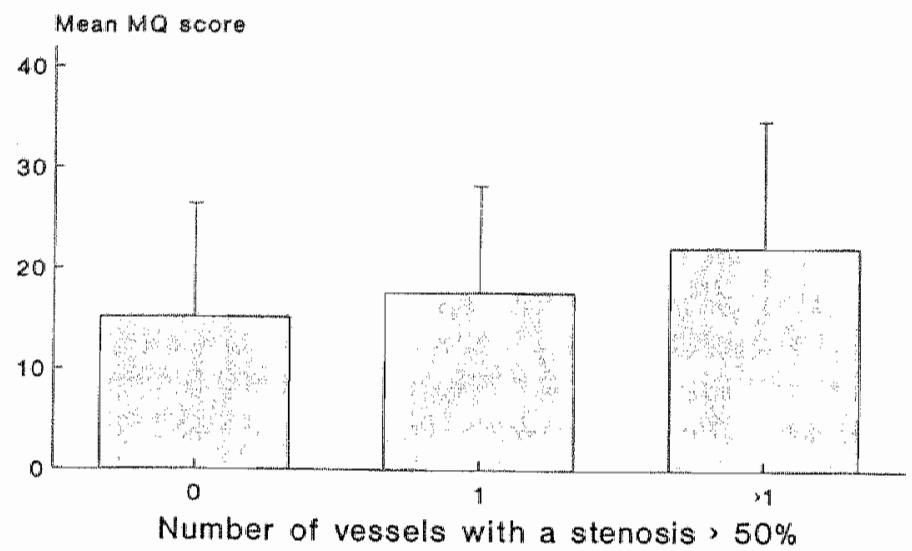

Figure 2. The relationship between vital exhaustion two weeks after PTCA and the extent of coronary artery disease after PTCA. Extent of coronary disease after PTCA reflects the number of coronary arteries which were not dilated during PTCA and hence continued to be significantly narrowed (i.e., stenosis $>50 \%$ ). The MQ at two weeks after discharge is not significantly related to the extent of coronary artery disease after PTCA $(F=1.9, p=0.16)$. Patients free of any lesion $(N=68)$ had a MQ score of $15.2 \pm$ 11.2, while those with one $(\mathrm{N}=30)$ or more than one narrowed vessel $(\mathrm{N}=10)$ had $\mathrm{MQ}$ scores of $17.6 \pm 10.6$ and $22.1 \pm 12.5$, respectively.

\subsection{Discussion.}

The aim of the present study was to investigate whether restoration of coronary perfusion by coronary angioplasty is followed by a substantial reduction of feelings of exhaustion. A positive association was found between exhaustion and the extent of coronary artery disease before PTCA, and on the average, feelings of exhaustion improved after PTCA. Both observations are in line with the contention that impaired coronary perfusion causes tiredness in patients with coronary disease. At the same time, however, only $4 \%$ of the variance of exhaustion scores was accounted for by the extent of atherosclerosis and $65 \%$ of the successfully treated patients were exhausted 
two weeks after PTCA. The severity of exhaustion was also evaluated six months after discharge, while patients who had repeat PTCA, bypass surgery, or myocardial infarction during that period were excluded. It appeared that there was no late decline in exhaustion scores. Moreover, there was no clear difference between patients who had typical anginal complaints at six months and those without typical angina. These results suggest that exhaustion in coronary patients can, at best, partially be explained by the presence of coronary atherosclerosis.

The modest magnitude of the relationship between the extent of coronary artery disease and vital exhaustion and some limitations of the study design give rise to the following questions: 1 . Is the association between the extent of coronary artery disease and exhaustion well estimated? 2. Is the change of vital exhaustion adequately evaluated? 3. What are the implications of the use of a healthy reference group? These questions will be addressed in turn.

The association between the extent of coronary artery disease and exhaustion before PTCA was statistically significant but the coefficient of determination $\left(R^{2}\right)$ was low. This may point to a possible statistical underestimation of an existing relationship. Patients without significant coronary lesions on admission were not included in this study. This leads to a restriction of range in the severity of coronary disease, which might artificially reduce $\mathbf{R}^{2}$ (Pedhazur, 1982). But after PTCA, the argument of restriction of range does not apply since a substantial number of patients are free of significant stenoses at that time.

Successful PTCA did not result in a substantial reduction of the number of patients reporting feelings of exhaustion. On admission, $75 \%$ of the patients were exhausted and after successful PTCA this percentage was still $65 \%$. Only 5 patients who were exhausted on admission improved substantially (i.e., a decrease from the third to the first tertile of the MQ). Furthermore, since the mean MQ score on admission was far above the vallues of the general population, the decrease of the MQ score may be a consequence of statistical regression to the mean. The latter argument is supported by the finding that the change in MQ score is solely predicted by the state of exhaustion on admission.

One might argue that the time between PTCA and the two weeks follow-up is too short, because at that time patients are still handling the emotional experience of hospitalization and have not yet resumed their daily activities. The question to be answered by this study addresses the effect of restoration of coronary blood-flow on exhaustion. It is known that restenosis of the dilated coronary arteries occurs in $20 \%$ to $30 \%$ of patients with an initially successful PTCA (Detre et al., 1989; Rupprecht, 1990), and that the incidence restenosis peaks at three months after the procedure (Serruys et al., 1988; Liu et al., 1989). The possible presence of new lesions would obscure the validity of the answer to the question at hand. Therefore, a follow-up assessment at two weeks after discharge (i.e. before the time at which restenosis occurs) was considered to be an appropriate time of evaluation. 
In order to assess the effect of PTCA on exhaustion, we have compared the exhaustion scores of the present sample with the distribution of the exhaustion scores in the general population. The present finding, that the number of exhausted individuals after PTCA hardly changed, could be related to the presence of disease in general and might be independent of coronary disease in particular. However, patients were used as their own controls in the present study, and in addition such an explanation would add up to the contention that exhaustion is not merely attributable to impaired coronary perfusion. Exhaustion scores were still increased after successful PTCA if compared with scores of patients with other diseases (means $16.5 \pm 11.3$ and $11.7 \pm$ 9.8 , respectively).

In the present study we limited ourselves to the severity and location of stenoses as measures of coronary artery disease. No information is available on transient ischemia, nor on collateral blood supply, which may have been of relevance for the presence of feelings of exhaustion. As far as cardiac pump function is concerned, it appeared that only four patients had a poor left ventricular function. Therefore, it seems that a decreased pump function of the heart is not a plausible candidate to explain exhaustion in these patients.

The main conclusion of this study is that excess fatigue in patients undergoing PTCA can only partially be explained by severity of atherosclerosis, use of medication, or duration and severity of anginal complaints. In addition, the number of exhausted patients did not markedly decrease after successful PTCA. The latter finding does not seem to correspond with the common clinical experience that most patients feel much better after PTCA. These positive reports of patients refer probably to the relief of anginal complaints and the fatigue on exertion and apparently not to the syndrome of vital exhaustion which is not restricted to tiredness alone, but also includes other aspects such as increased irritability and demoralization. This investigation does not give an answer as to the origin of exhaustion in coronary patients, and it does not prove that excessive fatigue is caused by prolonged psychological tension. The present study does suggest, however, that the association between vital exhaustion and coronary artery disease is not strong enough to explain the observed relationship between exhaustion and future myocardial infarction and sudden cardiac death. 


\section{Chapter 5.}

\section{Vital exhaustion predicts new cardiac events after successful coronary angioplasty.'}

Estimates of the prevalence of excess fatigue preceding cardiac morbidity and mortality vary between studies and range from $30 \%$ to $70 \%$ (see chapter 1 , secrion 1 for references). Most cardiology textbooks attribute this state to global coronary ischemia caused by clinical or subclinical heart disease, or alternatively, to prolonged psychological tension (Braunwald, 1988; Hurst et al., 1990). Although such statements are derived from careful clinical observations, it is noteworthy that there are virtually no systematic studies which have addressed the etiology of fatigue in persons at risk for manifest coronary artery disease.

Psychological analysis of patients reporting extreme tiredness before myocardial infarction revealed that this state is composed of three characteristics: 1) lack of energy; 2) increased irritability; and 3) feelings of demoralization (Appels, 1990). This state was labelled "vital exhaustion" and can be assessed by the Maastricht Questionnaire (Appels et al., 1987). Proof for the association between exhaustion and myocardial infarction was found in case-control studies and one prospective study (Appels \& Mulder, 1988; Falger, 1989). In the prospective study, 3877 healthy men were followed for 4.2 years. Exhaustion was associated with a relative risk of 2.28 of first myocardial infarction. Although the association was independent of the standard risk factors for myocardial infarction, it was possible that the feelings of exhaustion were merely a consequence of progressing atherosclerosis. which would explain its relationship with clinically manifest heart disease. Thus, the possible confounding by subclinical coronary artery disease could not be ruled out as an explanation for the observed relationship between the state of exhaustion and myocardial infarction.

The purpose of the study described in this chapter was to test the following: Vital exhaustion predicts new cardiac events after successful coronary angioplasty, independently of the extent of underlying coronary artery disease and other predictors of the clinical course after angioplasty (Hypothesis 4). We conducted a prospective study of a group of patients with successful Percutaneous Transluminal Coronary Angioplasty (PTCA), who were followed for recurrent coronary events. New coronary events after PTCA are usually the result of restenosis of the dilated area. Restenosis occurs in $20 \%$ to $30 \%$ of all patients after initial successful PTCA (Detre et al., 1989; Serruys

This chapter is in press: Willem J. Kop, Ad P.W.M. Appels, Carlos F. Mendes de Leon, Hans B. de Swart, Frits W. Bär. Pshchosom Med. 
et al., 1990; Rupprecht et al., 1990). Since the state of the coronary arteries at the time of PTCA is well documented, this group of patients provides a unique opportunity to test the current hypothesis.

\subsection{Methods.}

\subsubsection{Patients.}

Consecutive patients referred for coronary angioplasty were recruited between August 1988 and December 1989. Before recruitment patients had to give informed consent. To be included in the study, it was required that patients had an angiographically documented stenosis of more than $70 \%$ in at least one coronary artery, and that they were symptomatic (i.e., angina class II-IV according to the New York Heart Association classification). In addition, patients had to meet the following criteria: 1) $\leq 70$ years old; 2) no previous revascularization by PTCA or coronary artery bypass grafting; 3) no valvular or other cardiac disorders; and 4) no severe co-morbidity, such as cancer or psychiatric disorder (see also, chapter 2, section 1.4.).

For the purpose of this study, only patients with a successful dilatation in at least one coronary artery were included. A successful dilatation was defined as a definite reduction of the stenosis from over $70 \%$ before PTCA to a residual stenosis of less than 50\% after PTCA. Successful dilatation was achieved in 167 of the 196 eligible patients (success rate $85 \%$ ). Of these, five patients were excluded because they suffered myocardial infarction due to the PTCA and fourteen were excluded because of recurrent chest pain within two weeks after PTCA (Table 1 ). The latter fourteen patients were excluded for two reasons: 1) to ensure that the assessment of vital exhaustion, which took place two weeks after discharge (see below), was not in any way affected by the presence of anginal complaints; and 2) in order to preserve the chronological order between the assessment of vital exhaustion and the development of new events.

Patients completed the Maastricht Questionnaire (MQ) to evaluate vital exhaustion. The MQ is a self-report questionnaire consisting of 21 items with a score range from 0 to 42 and a good internal consistency (Crombach"s $\alpha=0.89$ ). The mean MQ score in a healthy population is $8.8( \pm 8.7)$ (Appels et al., 1987). The development of the MQ began with the selection of an initial item-set based on clinical interviews with coronary patients. Subsequently, only those items were included that discriminated myocardial infarction patients from controls in a case-control study (Appels et al,, 1980). Next, the validity of these items was investigated in a prospective study (Appels et al., 1987; 1989). It appeared that healthy men with scores in the upper tertile of the MQ were at elevated risk to suffer a first myocardial infarction. The estimated relative risk of myocardial infarction was 2.28 during a mean follow-up of 4.2 years (Appels \& Mulder, 1989). The association between vital exhaustion and myocardial infarction was most apparent in the first year of follow-up and gradually 
decreased during subsequent years (estimated relative risks for each year were 10.05, $2.23,3.04$, and 0.68 , respectively) (Appels \& Mulder, 1988). The results of this studly were confirmed in a case-control study (Falger, 1989) in men, and recently the predictive value of vital exhaustion for first myocardial infarction was validated in women (Appels et al., in press). The content of the MQ has been published by Appels et al. (1987) and is provided in appendix II. The MQ was mailed two weeks following discharge from the hospital. Twenty-one patients failed to return a valid questionnaire (i.e., more than five items were not answered) or returned the questionnaire too late, that is, after more than one month following discharge. This resulted in the present study sample of 127 patients.

Table 5.1. INCLUSION AND EXCLUSION CRITERIA.

\section{Inclusion criteria:}

Informed consent

Stenosis $\geq 70 \%$ suitable for PTCA

Symptomatic

Age $\leq 70$ years

No previous revascularization

No severe (cardiac) co-morbidity

Total mumber of eligible patients

Exclusion criteria:

Failed PTCA

Myocardial infarction during PTCA

Recurrent angina within 2 weeks

\subsubsection{Patient characteristics.}

The number of diseased coronary arteries was used as an indicator of the extent of coronary disease. Vessels were categorized into: right coronary artery (RCA), left anterior descending artery and diagonal branches (LAD), and circumflex artery (CX). Two measures of the extent of coronary artery disease after successfull PTCA were used: the number of undilated lesions that remained with a stenosis $>50 \%$, and the severity of residual stenosis of the successfully dilated artery. Remaining stenoses $>$ $50 \%$ could occur in case of multiple lesions, where only the culprit lesion was dilated.

The number and location of lesions before and after PTCA are presented in Table 
2. Significant stenoses remained in the RCA in 22 patients (25 lesions), in the LAD in 24 ( 27 lesions), and in the CX in 25 patients ( 27 lesions). Of the 127 patients with successful PTCA, there were 49 patients who continued to have a significant stenosis in one or more coronary arteries (33 in one, 14 in two, and 2 in three arteries). The median residual stenosis of the successfully dilated lesion was $20 \%$ (range $0 \%-50 \%$; mean $22.2 \pm(1.4)$.

\section{Table 5.2. NUMBER AND LOCATION OF CORONARY LESIONS BEFORE AND AFTER SUCCESSFUL PTCA.}

\begin{tabular}{|c|c|c|c|}
\hline $\begin{array}{l}\text { Severity of coronary } \\
\text { disease on admission }\end{array}$ & $\mathbb{N}$ & $\begin{array}{l}\text { Number of significant } \\
\text { lesions before PTCA }\end{array}$ & $\begin{array}{l}\text { Number of significant } \\
\text { lesions after PTCA }\end{array}$ \\
\hline 1 vessel disease & 76 & 87 & 7 \\
\hline 2 vessel disease & 41 & 98 & 45 \\
\hline 3 vessel disease & 10 & 36 & 24 \\
\hline Total & 127 & 221 & 76 \\
\hline Location of the lesion & $\mathbb{N}$ & $\begin{array}{l}\text { Number of significant } \\
\text { lesions before PTCA }\end{array}$ & $\begin{array}{l}\text { Number of } \\
\text { dilated areas }\end{array}$ \\
\hline Right Coronary artery & 60 & 68 & 43 \\
\hline Left Anterior Descending & 84 & 104 & 80 \\
\hline Circumflex & 44 & 49 & 22 \\
\hline Total & & 221 & 145 \\
\hline
\end{tabular}

Left ventricular function was evaluated by echocardiography or angiography. A distinction was made between those who had a deteriorated left ventricular function resulting in a left ventricular ejection fraction $<40 \%$ and those with a good left ventricular function.

To assess the impact of complaints before admission on the subsequent clinical course, the importance of the duration and severity of angina was investigated. The diagnosis upon which PTCA was indicated was also taken into account, making a distinction between stable angina, unstable angina, and myocardial infarction. Unstable angina was defined as angina at rest, progressive angina, or recent onset angina. The possible influence of myocardial infarctions occurring more than one month before hospitalization) was registered and statistically controlled for. Information about these variables was obtained form the medical record.

The classical risk factors for coronary disease were investigated as far as history of hypertension, diabetes mellitus, and smoking were concerned. The variable smoking was categorized into: 'never smoked', 'stopped smoking more than one year before 
PTCA', 'stopped smoking less than one year before PTCA' and 'smoking until admission', using interview collected information while the patient was hospitalized. The contribution of peripheral vascular disease was also examined.

\subsubsection{Follow-up.}

The occurrence of new cardiac events was recorded during a follow-up of 1.5 years after PTCA. All events had to be the consequence of new coronary ischemia. Data were obtained from the outpatient charts. A new cardiac event was defined as one of the following endpoints: 1) cardiac death; 2) myocardial infarction; 3) coronary bypass surgery; 4) repeat PTCA; 5) new coronary lesion; or 6) new angina with documented ischemia by thallium scintigraphy or ST segment changes during exercise. The category 'new coronary lesion' consisted of a) 'restenosis' of the dilated artery to a lesion $>50 \%$, b) 'progression of coronary disease' which could be a new significant lesion (>50\%) or increase in an already existing lesion by $20 \%$ or more. Note that a repeat angiogram was only made on clinical indication. If none of these events occurred, the follow-up was considered to be event-free. Information on follow-up status was complete for all patients.

\subsubsection{Statistical analysis.}

Univariate associations between the independent variables and new cardiac events were examined using $\mathrm{Chi}^{2}$ tests. The Kaplan-Meier curve was used in order to investigate the predictive value of vital exhaustion over time. Patients were divided into a 'vitally exhausted' group (with MQ scores in the highest tertile of the MQ) and a 'not exhausted' group (with MQ scores in the لower two tertiles).

The predictive value of vital exhaustion, controlled for the other predictors of the clinical course, was evaluated by multiple logistic regression analyses (Kleinbaum \& Kupper, 1979). The backward stepwise procedure was used with likelihood ratio testing. In the first analysis, variables were entered which showed a significant univariate association $(p<0.1)$ with a new cardiac event. Secondly, an analysis was performed entering all theoretically relevant independent variables as well as vital exhaustion.

For all patients who had a second coronary angiogram, the t-test was used to investigate whether patients who developed restenosis or progression of coronary disease had different exhaustion scores from those who remained to be free of new lesions. 
chapter $\$$

\subsection{Results.}

\subsubsection{Clinical predictors of new cardiac events.}

The mean age of the patients was $55.6 \pm 9.1$ (range 33 to 70 ). Twenty-two (17\%) were females. Non-response to the MQ at two weeks after discharge was not related any of the clinical characteristics $(\mathrm{p}>0.1)$.

New cardiac events occurred in 29 patients (23\%). One patient died of myocardial infarction, one survived a myocardial infarction, four patients were referred for bypass surgery, eleven had a repeat PTCA, seven had increase of coronary artery disease but no rewascularization procedure, and five had new angina with documented coronary ischemia (Table 3).

\section{Table 5.3. RELATION BETWEEN VITAL EXHAUSTION} AND CLINICAL STATUS 1.5 YEAR AFTER PTCA.

\begin{tabular}{|c|c|c|c|c|}
\hline & \multicolumn{2}{|c|}{$\begin{array}{r}\text { Not exhausted } \\
(\mathrm{MQ} \leq 18)\end{array}$} & \multicolumn{2}{|c|}{$\begin{array}{r}\text { Exhausted } \\
(\mathrm{MQ}>18)\end{array}$} \\
\hline & $\mathbb{N}$ & $\%$ & $\mathrm{~N}$ & $\%$ \\
\hline Event-free & 70 & 83 & 28 & 65 \\
\hline New angina with ischemia & 3 & 4 & 2 & 5 \\
\hline New coronary lesion & 3 & 4 & 4 & 9 \\
\hline Repeat PTCA & 7 & 8 & 4 & 9 \\
\hline Coronary bypass surgery & 1 & 1 & 3 & 7 \\
\hline Myocardial infarction & & & 1 & 2 \\
\hline Cardiac death & & & 1 & 2 \\
\hline
\end{tabular}

Univariate statistics comparing patients with $(\mathrm{N}=29)$ and without $(\mathrm{N}=98)$ new cardiac events are presented in Table 4. Patients who had multi-vessel disease and those who had hypercholesterolemia were significantly over-represented in the group with new cardiac events. There was no relationship between the incidence of new cardiac events and the diagnosis on admission (i.e., stable angina, unstable angina, or myocardial infarction). In patients where the values of triglycerides and cholesterol were reported $(\mathrm{N}=88$ ), higher levels were found in those who suffered a new cardiac event during follow-up $(2.8 \pm 1.3 \mathrm{mmol} / 1$ and $7.1 \pm 1.4 \mathrm{mmol} / 1$, respectively) than in patients without a new event $(2.2 \pm 1.3 \mathrm{mmol} / \mathrm{l}$ and $6.4 \pm 1.2 \mathrm{mmol} / 1$, respectively; $\mathrm{p}<$ $0.07)$. Only one patient had insulin-dependent diabetes mellitus, and this patient had an event-free follow-up. As shown in Table 4, there were no differences in the severity of residual stenosis nor in the number of remaining lesions after PTCA. In patients with new cardiac events, the RCA was dilated in $24 \%$, the LAD in $69 \%$, and the CX in $21 \%$, while for those with an event-free follow-up these percentages were $32 \%, 53 \%$, and $18 \%$, respectively. 
Table 5.4: CLINICAL, ANGIOGRAPHIC AND DEMOGRAPHIC CHARACTERISTICS OF PATIENTS WITH OR WITHOUT A NEW CARDIAC EVENT AFTER SUCCESSFUL PTCA

\begin{tabular}{|c|c|c|c|c|c|c|c|c|c|}
\hline & & \multicolumn{2}{|c|}{ No Event } & \multicolumn{2}{|c|}{ Event } & \multicolumn{2}{|c|}{ Total } & \multirow[t]{2}{*}{ OR } & \multirow[t]{2}{*}{$\mathrm{p}$} \\
\hline & & $\mathrm{N}$ & $\%$ & $\mathbb{N}$ & $\%$ & $\mathrm{~N}$ & $\%$ & & \\
\hline \multirow[t]{2}{*}{ Vital exhaustion } & no & 70 & 71.4 & 14 & 48.3 & 84 & 66.1 & & \\
\hline & yes & 28 & 28.6 & 15 & 51.7 & 43 & 33.9 & 268 & 0.02 \\
\hline \multirow[t]{2}{*}{ Gender } & Male & 82 & 83.7 & 23 & 79.3 & 105 & 82.7 & & \\
\hline & Female & 16 & 16.3 & 6 & 20.7 & 22 & 17.3 & 1.34 & ns \\
\hline \multirow[t]{2}{*}{ Age } & $<55$ years & 47 & 48.0 & 15 & 48.8 & 62 & 48.8 & & \\
\hline & $>55$ years & 51 & 52.0 & 14 & 51.2 & 65 & 52.2 & 0.86 & $\mathrm{~ns}$ \\
\hline \multicolumn{10}{|c|}{ Duration of complaints } \\
\hline & $<1$ month & 33 & 33.7 & 12 & 42.9 & 45 & 35.7 & & \\
\hline & $>1$ month & 65 & 66.3 & 16 & 57.1 & 81 & 64.3 & 0.68 & ns \\
\hline \multirow[t]{3}{*}{ Angina class } & II & 30 & 30.9 & 6 & 20.7 & 36 & 28.6 & & \\
\hline & III & 44 & 45.4 & 14 & 48.3 & 58 & 46.0 & & \\
\hline & IV & 23 & 23.7 & 9 & 31.0 & 32 & 25.4 & 1.39 & ns. \\
\hline \multicolumn{2}{|l|}{ Stable angina } & 29 & 29.6 & 5 & 17.2 & 34 & 26.8 & & \\
\hline \multicolumn{2}{|l|}{ Unstable angina } & 48 & 49.0 & 20 & 69.0 & 68 & 53.5 & & \\
\hline \multicolumn{2}{|c|}{ Myocardial infarction } & 21 & 21.4 & 4 & 13.8 & 25 & 19.7 & 1.11 & ns \\
\hline \multicolumn{10}{|c|}{ History of myocardial infarction } \\
\hline & no & 75 & 76.5 & 20 & 69.0 & 95 & 74.8 & & \\
\hline & yes & 23 & 23.5 & 9 & 31.0 & 32 & 25.2 & 1.47 & ns. \\
\hline \multicolumn{10}{|c|}{ Vessel disease before PTCA } \\
\hline & 1 & 65 & 66.3 & 11 & 37.9 & 76 & 59.8 & & \\
\hline & 2 & 26 & 26.5 & 15 & 51.7 & 41 & 32.3 & & \\
\hline & 3 & 7 & 7.1 & 3 & 10.3 & 10 & 7.9 & 2.06 & 0.02 \\
\hline \multicolumn{10}{|c|}{ Left wentricular function } \\
\hline & Good & 80 & 81.6 & 24 & 82.8 & 104 & 81.9 & & \\
\hline & Poor & 4 & 4.1 & 1 & 3.4 & 5 & 3.9 & 0.83 & $n s$ \\
\hline & Not determine & 114 & 14.3 & 4 & 13.8 & 18 & 14.2 & & \\
\hline
\end{tabular}

Table 4 will be continued on the next page. 
chapter 5

Table 5.4. continued:

\begin{tabular}{|c|c|c|c|c|c|c|c|c|c|}
\hline & & \multicolumn{2}{|c|}{ No Event } & \multicolumn{2}{|c|}{ Event } & \multicolumn{2}{|c|}{ Totall } & \multirow[t]{2}{*}{ OR } & \multirow[t]{2}{*}{ p } \\
\hline & & $\mathrm{N}$ & $\%$ & $\mathbb{N}$ & $\%$ & $\mathbb{N}$ & $\%$ & & \\
\hline \multirow{2}{*}{ Hypertension } & tho & 78 & 79.6 & 21 & 72.4 & 99 & 78.0 & & \\
\hline & yes & 20 & 20.4 & 8 & 27.6 & 28 & 22.0 & 1.49 & ns \\
\hline \multicolumn{10}{|c|}{ Peripheral vessel disease } \\
\hline & no & 91 & 92.9 & 26 & 89.7 & 117 & 92.1 & & \\
\hline & yes & 7 & 7.1 & 3 & 10.3 & 10 & 7.9 & 1.50 & ns \\
\hline \multicolumn{10}{|c|}{ Hyperchalesterolemia } \\
\hline & no & 86 & 87.8 & 21 & 72.4 & 107 & 84.3 & & \\
\hline & yes & 12 & 12.2 & 8 & 27.6 & 20 & 15.7 & 2.73 & 0.05 \\
\hline \multicolumn{10}{|c|}{ Smoking status on admission: } \\
\hline Never & & 15 & 15.6 & 4 & 13.8 & 19 & 152 & & \\
\hline Stopped > & & 26 & 27.1 & 6 & 20.7 & 32 & 25.6 & & \\
\hline Stopped < & & 15 & 15.6 & 4 & 13.8 & 19 & 15.2 & & \\
\hline Continued & g until PTCA & 40 & 41,7 & 15 & 51.7 & 55 & 44,0 & 1.17 & ns \\
\hline \multicolumn{10}{|c|}{$\begin{array}{l}\text { Number of vessels with a lesion } \\
>50 \% \text { after PTCA }\end{array}$} \\
\hline & 0 & 64 & 65.3 & 13 & 44.8 & 77 & 60.6 & & \\
\hline & 1 & 23 & 23.5 & 11 & 37.9 & 34 & 26.8 & & \\
\hline & $\geq 2$ & 11 & 11.2 & 5 & 17.2 & 16 & 12.6 & 1.64 & ns \\
\hline Residual steno & eans \pm s.d.) & $22.5 \pm$ & 11.7 & $21.6 \pm$ & 10.4 & $22.3 \pm$ & 11.4 & & ns \\
\hline
\end{tabular}

5.2.2. The predictive value of vital exhaustion.

Vital exhaustion was significantly related to the occurrence of cardiac events during follow-up. Fifteen $(35 \%)$ of the 43 patients who had a $\mathrm{MQ}$ score in the third tertile (i.e., MQ $>18$ ) had a new cardiac event, while only $14(17 \%)$ of the 84 patients in the lower two tertiles experienced such an event (crude odds ratio $2.7 ; \mathrm{CI}=1.1$ $6.3 ; p=0.02$ ). Essentially the same relative risk was observed when the analysis was repeated for patients who did not have myocardial infarction on admission ( $R R=2.7$; $C I=1.1-6.8$ ).

The median time between the PTCA procedure and event was 17 weeks (4 months). The Kaplan-Meier curve comparing exhausted and not exhausted patients is shown in Figure 1. The clinical course of exhausted patients is significantly less favorable $\left(\mathrm{Chi}^{2}{ }_{\text {iBreslowi }}=5.2 ; \mathrm{p}=0.02\right)$. 


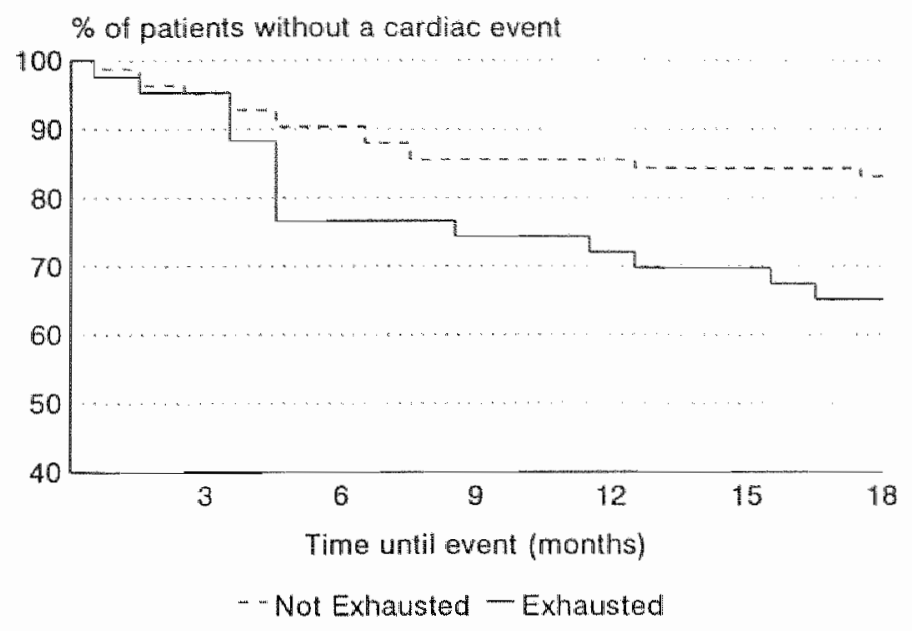

Figure 5.1. The relationship between vital exhaustion and new cardiac events during 1.5 years follow-up, tising the Kaplam-Meier curve to compare Exlrausted $(\mathrm{N}=43)$ and $\mathrm{Not}$ Exhausted $(\mathrm{N}=84)$ patients. The $y$-axis shows the proportion of patients without a cardiac event; the abscissa the duration of follow-up after coronary angioplasty. "The exhausted group (solid line) had a less faworable clinical course than the not exhausted group (diashed line) $(\mathrm{p}=0.02)$.

Multiple logistic regression analysis showed that when the sample specific predictors of new cardiac events were controlled for simultaneously (i.e., hypercholesterolemia, multi-vessel disease before PTCA, and vital exhaustion), all three variables were kept in the model as predictors of new cardiac events (Table 5). The second multiple logistic regression analysis included all variables which might theoretically influence the clinical course after PTCA. These are: age, sex, history of myocardial infarction, hypertension, peripheral vascular disease, hypercholesterolemia, smoking status, severity of angina on admission, diagnosis of stable, unstable angina, or myocardial infarction, multivessel disease on admission, left ventricular function, the number of remaining lesioned vessels after PTCA, and severity of residual stenosis. Results were marginally different from the previous multiple logistic regression analysis; multivessel disease, hypercholesterolemia, and vital exhaustion were the only variables which were kept in the model.

A new coronary angiogram was performed on clinical grounds only. Twenty-eight patients were evaluated this way, of whom 10 had restenosis of the dilated segment, 5 had progression of coronary artery disease, and 7 had both restenosis and progression of coronary disease. Six patients did not have a significant increase in coronary artery disease. Patients who had a new coronary angiogram during follow-up, tended to be more vitally exhausted than those who did not receive such an investigation (means 
$18.8 \pm 12.2$ and $14.5 \pm 10.7, t=1.83, p=0.07$ ). However, patients who had restenosis or progression of coronary disease at re-angiography were not significantly different from those who did not show new coronary lesions (MQ means: $17.1 \pm$ $10.4,16.8 \pm 12.3$ and $20.8 \pm 18.4$, respectively).

\section{Table 5.5. MULTIPLE LOGISTIC REGRESSION ANALYSIS \\ WITH SAMPLE SPECIFIC PREDICTORS \\ OF NEW CARDIAC EVENTS.}

\begin{tabular}{lrrrr}
\hline & B & s.e. & OR & p \\
& & & & \\
Vital exhaustion & 0.85 & 0.46 & 2.340 .06 \\
Multivessel disease & 1.04 & 0.45 & 2.840 .02 \\
Hypercholesterolemia & 1.01 & 0.56 & 2.750 .07 \\
\hline
\end{tabular}

\subsection{Discussion.}

The principal finding of this study is that patients who are exhausted early after PTCA are more likely to suffer new cardiac events during 1.5 year follow-up than patients who are not vitally exhausted. This association cannot be attributed to remaining stenoses, anginal complaints or other clinical characteristics. Thirty-five percent of the patients who were exhausted after PTCA experienced a new cardiac event, while only $17 \%$ of those who were not exhausted suffered such an event (OR $=2.7$ ).

One of the exclusion criteria was the recurrence of anginal complaints within two weeks after successful PTCA. There were two reasons for this strategy. First, it is possible that the assessment of vital exhaustion is biassed by the presence of angina. Second, it is difficult to determine if these complaints are the result of an unsatisfactory result of the PTCA or if they are caused by early restenosis.

One possible interpretation of the increased risk of vital exhaustion for new cardiac events is that patients who are exhausted are more likely to visit their general practitioner or cardiologist (e.g., Costa et al., 1985). This may lead to an increased detection rate of cardiac events and also to more readmissions (Rothman, 1986). However, all patients in the present study were required to attend the outpatient clinic for follow-up care on a regular basis. Therefore, it is not likely that the detection rate of new cardiac events is influenced by a physician-based referral bias. Increased selfreferral may have occurred more often in some of the exhausted patients. Angiograms during follow-up were only made when anginal complaints increased. In six of the twenty-eight patients with a repeat angiogram, no restenosis or progression of coronary artery disease was observed. This group had elevated exhaustion scores compared with patients who did not have a repeat angiogram. Because the result of angiography was negative with respect to progression of coronary disease, these 
patients were allocated to the event-free group. In addition, the data show that the more severe cardiac events (i.e., repeat PTCA, bypass surgery, myocardial infarction, or cardiac death) occurred more often in the vitally exhausted than in the nonexhausted patients ( $20 \%$ versus $9 \%$ ). Therefore it is not likely that referral bias can explain the observed relationship between exhaustion and the clinical course after PTCA.

New cardiac events after successful PTCA are assumed to be caused by late restenosis of the dilated location (Serruys et al., 1988; Liu et al., 1989). The exact pathophysiological mechanism of this phenomenon is not fully understood, but it is probable that endothelial damage at the site of balloon dilatation induces an excessive fibrocellular reaction (see also chapter 7, section 2). The present study did not reveal differences in the severity or the number of remaining stenoses between patients with or without a new cardiac event. Furthermore, restenosis is not always accompanied by recurrence of anginal complaints, and new angina may also be caused by other lesions (Bengtson, 1990). One shortcoming of the present study is that re-angiography was only available for a limited number of patients with cardiac complaints.

A poor left ventricular function was observed in only five patients of whom one suffered a new cardiac event. It is important to note that those who had a poor left ventricular contraction pattern were not more exhausted than patients who had a normal left ventricular function. Therefore, it is unlikely that a decreased cardiac output can explain the presence of vital exhaustion as measured two weeks after PTCA nor that the low cardiac output accounts for the predictive value of vital exhaustion for new cardiac events.

There is considerable congruency between the characteristics of vital exhaustion and depression (see chapter 1, section 4.1.). The results of the present study could be interpreted as a replication of the observation of Carney et al. (1988), who showed that depression is of predictive value in the clinical course of coronary disease. Although it is beyond the scope of this chapter to compare both concepts in detail, a few differences are worth mentioning. First, the core characteristic of depression is the affect disturbance. In this respect it is important to note that exhausted persons rarely complain about sadness, low self-esteem, or feelings of guilt (Appels, 1990). This suggestion was supported in a study by van Diest (1991), where normal and exhausted subjects were evaluated with the Profile of Mood States. It was found that exhausted subjects were more fatigued and were less vigorous than not exhausted subjects, but there was no difference on the depression sub-scale. Therefore, we believe that vital exhaustion is conceptually different from depression.

The question as to the mechanism of the relationship between vital exhaustion and a negative clinical course after PTCA can not be answered by this study. Van Doornen (1988) showed that cholesterol levels increased during stress in healthy subjects who were exhausted. The data presented in chapter 6 indicate that there may be a reduced fibrinolytic activity in exhausted individuals. These findings may indicate that exhausted patients are more prone to develop atherosclerotic plaques or thrombi, 
leading to coronary artery stenosis or occlusion. Alternatively, one might speculate that vital exhaustion is associated with an increased vasomotor tone. Ardissino et al. (1991) observed a high risk for restenosis after successful PTCA in patients with hyperventilation-induced abnormal coronary vasoconstriction. It may be that exhausted persons share common characteristics with those who have a positive hyperventilation test. The issue of the psychobiological mechanism will be discussed in more detail in chapter 7.

The present study shows that vital exhaustion is of relevance in the prediction of the clinical course after PTCA. The relationship between vital exhaustion and new cardiac events continued to be present when the severity of coronary artery disease was controlled for. Moreover, it is shown in the previous two chapters that less than $5 \%$ of the variance of MQ scores can be explained by the severity of coronary disease, and that clinical characteristics (among which: angina, dyspnea and duration of complaints) do not sufficiently explain vital exhaustion either. Therefore, it may be possible that vital exhaustion in patients with coronary disease has, at least in part, a psychological origin. It is a tentative hypothesis that the risk for new cardiac events after PTCA has multifactorial determinants. If vital exhaustion can be reduced by psychological intervention, the long term result of PTCA may be influenced in a positive direction. This line of reasoning appears to be attractive, since pharmacological intervention trials have failed to substantially decrease cardiac incidents after PTCA (Hermans et al., 1991). Several psychological intervention studies have been applied to ameliorate the clinical course after myocardial infarction (e.g., Kallio et al., 1979; Friedman et al., 1986; Frasure-Smith \& Prince, 1989). with results justifying further application and investigation. Since $20 \%-30 \%$ of patients with PTCA are readmitted within one year after coronary angioplasty, this high incidence deserves our attention (Markovitz \& Matthews, 1991). It may be that psychological stress reduction, resulting in an amelioration of feelings of vital exhaustion, will reduce the risk for new cardiac events in patients after PTCA. 


\section{Chapter 6.}

\section{The consequences of vital exhaustion for blood coagulation and fibrinolysis.'}

Acute physical and psychological stressors are known to influence the coagulation and fibrinolytic systems of the blood (Friedman et al., 1958; Cohen et al., 1988, Jern et al., 1989). Cannon (1914) already observed that emotional distress substantially reduced blood-clotting time in the cat, indicating a possible relationship between psychological stress and hypercoagulability. Several prospective studies have shown that prolonged psychological stress precedes myocardial infarction (Ruberman et al, 1984; Crisp et al., 1984; Reed et al., 1989; Rosengren et al. 1991). Continuous psychological stress may end in a state of exhaustion (Selye, 1977). In this respect, it is of relevance to note that excessive fatigue and tiredness are among the most prevalent premonitory symptoms of myocardial infarction (Kuller et al., 1972; Fraser, 1978; Klaboe et al., 1987). In a prospective study in 3877 healthy males, feelings of exhaustion appeared to be an independent predictor of first myocardial infarction (Appels et al., 1987). The typical characteristics of this state of exhaustion are: 1) lack of energy, 2) increased irritability, and 3) demoralization (Appels, 1990; see also chapter 1 (or a more extensive discussion).

The contribution of coagulation factors and fibrinolysis to the development of acute myocardial infarction is supported by a number of epidemiological investigations (Wilhelmsen et al., 1984; Stone, et al., 1985; Meade et al., 1986; Kannel et al., 1987; Hamsten et al., 1987; Anzar et al., 1988). There is a dynamic balance between the coagulation factors that generate fibrin and the removal of fibrin by the fibrinolytic system. Normally, both aspects of the cloting process are activated by stress, resulting in a balanced reaction. Most studies that addressed the influence of stress on blood-clotting investigated the effects of acute experimental stressors. For example, Jern et al. (1989) used physical exercise, adrenalin infusion, and a mental effort task (the Stroop test and mental arithmetic) as stressors. They observed an acute phase reaction, with an increase in clotting factors (von Willebrand factor, factors VII and VIII) and indicators of fibrinolysis (tissue plasminogen activator activity, and plasminogen activator antigen) in all three stressful conditions. Fibrinogen levels were also found to be higher following stress. Since mental stress had the same effects on hemostasis as did adrenalin infusion and physical exercise, Jern at al. postulated that an adrenergic mechanism is involved in the relationship between psychological stress

A modified version of this chapter is submitted by: Kop WJ, Hamulyák K, Pernot C, Appels A. 
and coagulation and fibrinolysis. In this respect, it is of interest that Gunn and colleagues (1967) noticed that electrical stimulation of the hypothalamus and the reticular formation resulted in a subsequent increase in plasma concentration of factor VIII. This supports the contention that the central nervous system is involved in the activation of factors that are of relevance in clot formation.

The relationship between chronic psychological stress and blood-clotting has, to our knowledge, never been investigated. There is some evidence that in case of sustained psychological arousal, clotting increases (factor VIII and fibrinogen) at the onset of a stressfull task, and decreases again over time (Palmblad el al., 1977). However, this was an experimental stressful situation with a duration of three days, which is obviously different from chronic psychological stress of daily life. Another observation in support of a relationship between continuous psychological stress and hematologic parameters is that people with high job-strann and of low social class have relatively high levels of fibrinogen and factor VII (Markowe et al., 1985). Social class and job-strain are rather crude measures of psychological stress, however. Therefore, the present study investigates if Vital exhaustion is associated with coagulation and fibrinolytic factors that promote clot-formation and stabilization (hypothesis 5, chapter 1).

\section{1. Methods.}

\subsubsection{Selection of subjects.}

The study was approved by the medical ethical committee of the University Hospital of Maastricht. Exclusion criteria were: age above 70 or below 35 years, coronary artery disease, hypertension, diabetes mellitus, thyroid disease, or any other chronic disease, treatment for psychiatric disorder, alcohol or other substance abuse, and current use of medication. Because age is associated with higher levels of factor VIIc and plasminogen activator inhibitor (PAD), the groups were matched on this variable (Brozovic at al., 1974; Andreotti et al., 1988).

Male subjects were invited to participate by means of a mailed questionnaire. The inventory included the Mastricht Questionnaire to assess feelings of exhaustion (Appels et al, 1987) and a short health status questionnaire. Next, potentially eligible subjects were invited to the hospital for clinical investigation to confirm the presence of exhaustion and to validate the absence of the exclusion criteria. This procedure resulted in 15 chronically stressed subjects, and 15 non-stressed controls (see also chapter 2, section 2).

Following the psychological assessment, blood-samples of each subject were collected on two occasions. The first sample was collected within two weeks after the interview. The second sample was obtained within three months of the first examination to correct for intra-individual fluctuations of the coagulation and fibrinolytic variables. 


\subsubsection{Blood collection.}

All blood specimens were collected between 8.30 and $10.00 \mathrm{a} . \mathrm{m}$. after an overnight fast, when the patient was at rest for at least 15 minutes. Venipuncture was performed with a $1.2 \mathrm{~mm}$ needle and minimal stasis was applied to the upper arm. Serum was prepared for the blood chemistry determinations. EDTA blood was used for routine hematological screening. The samples were immediately centrifuged at $4^{\circ} \mathrm{C}$ and aliquots of platelet free plasma were snap frozen and stored at $-70^{\circ} \mathrm{C}$ until further analysis.

\subsubsection{Measurements.}

The initial assessment of vital exhaustion as an end stage of prolonged psychological stress was by means of the Maastricht Questionnaire (MQ). In a healthy population the mean of the $\mathrm{MQ}$ is 8.8 (s.d. $=8.7$; possible score range $=0-42$ ). Patients were identified as exhausted if the score was $\geq 16$, and as not-exhausted if the MQ score was $\leq 6$. Eligible subjects were invited to the hospital for an interview by a clinical psychologist.

The clinical interview was used to confirm that the feelings of exhaustion were the consequence of a breakdown in adaptation to prolonged psychological stress. It was required that at least one of the elements of exhaustion (lack of energy, irritability, or demoralization) showed an increase in intensity during the last year before examination. By this procedure we could exclude false positives of the questionnaire, as well as subjects with complaints of exhaustion as the result of a psychiatric disorder (e.g., major depression). For this purpose an abbreviated version of the Diagnostic Interview Schedule was used (Robins et al., 1982; Bouman, 1987). As an overall measure of psychological stress, the Symptom Check List (SCL-90) was added (total score range 0 - 360; normal population mean 117.2 \pm 27.3 ; Derogatis, 1977; Arrindell \& Ettema, 1986).

Blood chemistry parameters were measured using a Dimension automate (Dupont; U.S.A.) including the level of cholesterol, triglycerides, creatinine, alkaline phosphatase, gamma GT, ALAT, ASAT, LDH, and bilirubin. Routine hematological parameters were measured immediately after blood-sampling using the $\mathrm{H} 1$ automate (Bayer; U.S.A.) including hematocrit value, platelet count, leucocyte count and differential.

The activity of coagulation factors VIIc and VIIIc was measured with a one-stage clotting assay using immuno-depleted factor deficient reagents obtained from Instrumentation Laboratory (Italy). Data are presented as percentages of values found with normal pool plasma. Fibrinogen was assessed in accordance with the method of Clauss (1957). PAl activity was measured with a chromogenic assay from Kabi (Sweden) in accordance with the manufacturer's prescription; tissue plasminogen activator (t-PA) antigen with an ELISA technique using a kit from Innogenetics (Belgium). All assays were analyzed in one run to prevent inter-assay variation. The laboratory personnel 
did not know the psychological status of the subjects.

Increased factor VII, fibrinogen, and $\mathrm{PAI}$ levels are related to smoking status $\mathrm{Kluft}$ et al., 1990; Hultin, 1991). We differentiated between non-smokers, subjects who stopped smoking more than two years ago, subjects who stopped during the last two years, and those who were smokers at the time of investigation.

\subsubsection{Statistical analysis.}

The results of the coagulation and fibrinolytic measurements were averaged over the two occasions of sampling for each subject separately. This procedure was applied in order to correct for intra-individual fluctuations of these variables. The comparison between chronically stressed and the non-stressed subjects was analyzed by the MannWhitney $U$ test on these averaged scores. Control variables were analyzed by the appropriate univariate tests (t-lest or Mann-Whitney).

\subsection{Results.}

There were no significant differences in the control variables between chronically stressed and non-stressed subjects (Table I). The mean MQ score in the chronically stressed group was $23.8 \pm 8.0$, and in the non-stressed group $3.5 \pm 1.9$. There was also a significant difference in the SCL-90 total score (means $149.9 \pm 39.4$ and 104.8 \pm 9.4 , respectively), validating that the chronically stressed group indeed had more psychological complaints $(t=4.13 ; p=0.001)$. No differences were found between the chronically stressed and the non-stressed groups in blood pressure, cholesterol and triglycerides, nor in any of the other laboratory measurements.

Table 6.I. THE RELATIONSHIP BETWEEN VITAL EXHAUSTION AND CONTROL VARIABLES.

\begin{tabular}{|c|c|c|c|c|c|}
\hline & \multicolumn{2}{|c|}{ non-stressed } & \multicolumn{2}{|c|}{ chronically stressed } & \\
\hline & Mean & s.d. & Mean & s.d. & \\
\hline Age & 54.2 & 9.7 & 49.3 & 7.3 & years \\
\hline Systolic BP & 120.3 & 12.7 & 121.7 & 17.3 & $\mathrm{mmHg}$ \\
\hline Diastolic BP & 77.7 & 10.0 & 78.7 & 7.4 & $\mathrm{mmHg}$ \\
\hline Cholesterol & 6.06 & 0.90 & 6.47 & 0.89 & $\operatorname{mmol} / 1$ \\
\hline Triglycerides & 1.47 & 1.03 & 1.96 & 0.94 & mmol// \\
\hline
\end{tabular}

continued on next page. 
Table 6.I. continued

\begin{tabular}{|c|c|c|c|c|c|}
\hline & \multicolumn{2}{|c|}{ non-stressed } & \multicolumn{2}{|c|}{ chronically stressed } & \\
\hline & Mean & s.d. & Mean & $s . d_{x}$ & \\
\hline Hematocrit & 0.44 & 0.03 & 0.45 & 0.03 & $1 / 1$ \\
\hline Thrombocyte & 232.8 & 71.6 & 233.3 & 47.9 & $n^{-1}$ \\
\hline Neutrophils & 53.6 & 7.9 & 56.3 & 7.1 & $\%$ \\
\hline Creatinine & 84.7 & 8.3 & 81.6 & 13.7 & $\mu \mathrm{mol} / 1$ \\
\hline Alk. phosphatase & 67.9 & 20.9 & 80.7 & 28.3 & $\mathrm{w} / 1$ \\
\hline gamma GT & 23.1 & 9.5 & 28.3 & 13.5 & $\mathrm{u} / 1$ \\
\hline ASAT & 19.9 & 3.4 & 18.5 & 4.1 & $w / 1$ \\
\hline ALAT & 27.7 & 5.6 & 28.5 & 5.1 & $u^{\prime} l$ \\
\hline $\mathrm{LDH}$ & 316.5 & 54.2 & 323.4 & 63.3 & $\mathrm{w} / \mathrm{l}$ \\
\hline Bilirubin & 13.5 & 8.5 & 10.3 & 2.1 & $\mu \mathrm{mol} / 1$ \\
\hline Smoking status & $\mathbf{N}$ & $\%$ & $\mathrm{~N}$ & $\%$ & \\
\hline never & 4 & 26.7 & 4 & 26.7 & \\
\hline stopped $>2$ years & 5 & 33.3 & 5 & 33.3 & \\
\hline stopped $\leq 2$ years & 2 & $\$ 3.3$ & 0 & 0.0 & \\
\hline currently smoking & 4 & 26.7 & 6 & 40.0 & \\
\hline
\end{tabular}

None of the differences between the groups was statistically significant.

Figure 1 shows that PAI levels were higher in the chronically stressed group than in the non-stressed subjects. The values of the chronically stressed group were in the upper range of the normal values of PAI. There were no statistically significant differences between the groups in coagulation factors VIIc and VIIIc, nor in fibrinogen and $\mathrm{t}-\mathrm{PA}$ antigen.

\subsection{Discussion.}

The principal finding of the present study is that significantly higher PAI levels are found in a well-defined group of subjects with chronic psychological stress than in non-stressed controls (median PAI activity 13.0 and 6.0 au/1, respectively). This is indicative of a reduced fibrinolytic capacity in individuals who are exhausted because of prolonged psychological stress. The importance of this and other fibrinolytic measures is supported by several studies which showed that increased PAI is associated with an unfavorable prognosis in unstable angina (Munkvad et al., 1990) or after successful coronary angioplasty (Kirschstein, 1989). The current study did not reveal an association between prolonged psychollogical stress and coagulation factors VIIc and 
VIIIc. Acute physiological as well as psychological stressors increase both coagulation and fibrinolytic activity. This study indicates that there may be an imbalance between coagulation and fibrinolysis in subjects who are chronically stressed. Such an interpretation is in line with the suggestion by Jern and colleagues (1989), who state that a disequilibrium between the coagulation and fibrinolytic systems in response to prolonged psychological stress may promote the risk for thrombus formation and hence myocardial infarction.

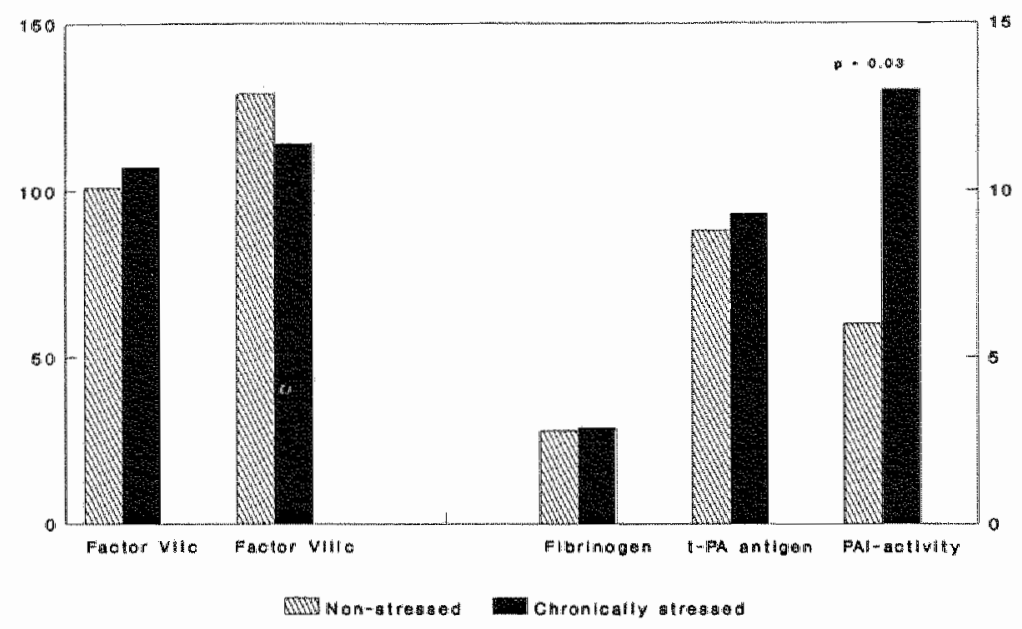

Figure 7.1. Coagulation factors and fibrinolysis as related to prolonged psychological stress leading to vital exhaustion. Factors VIIs and VIIlc are in $\%$, fibrinogen in $g r / l$, tissue plasminogen activator ( $t-P A)$ antigen in $n g r / m l$, and plasminogen activator inhibitor (PAI) activity in au/ml. PAl activity levels were higher in the chronically stressed group $(p=0.03) ;$ none of the other differences reached statistical significance $(p>0.1)$.

Smoking status may have confounded the present findings, since it is associated with factor VIIc, fibrinogen, and PAI (Kluft et al, 1990; Huttin, 1991). When current smokers were excluded from the sample, the analysis showed approximately the same results. There were no differences in coagulation factors and $t-P A$ antigen, and PAI activity was again higher in the exhausted group than in the not exhausted group (medians 5 and $13 \mathrm{au} / \mathrm{ml}$, respectively).

Coagulation and fibrinolysis may reflect an acute reaction to stressors. However, it is not likely that the higher PAI activity level in the chronically stressed group is attributable to differences in the acute phase reaction since no distinction between the groups was observed in platelet count. The absence of a relationship between chronic 
psychological stress and coagulation factors VIIe and VIIIe, fibrinogen, and t-PA antigen may be related to lack of statistical power in the present study due to the small sample-size. There was some support for factor VIIc to be rellated to chronic stress, because the data of the second occasion of blood-sampling showed significantly higher factor VII in the stressed group (107\%, versus $97 \% ; \mathrm{p}<0.01)$. But the present study suggests that fibrinolysis appears to be more influenced by chronic psychological stress than coagulation. This finding corresponds with the observation that in the setting of unstable angina pectoris, the fibrinolytic capacity is of substantially more predictive value for the development of myocardial infarction than thrombin activity (Munkvad et all, 1990).

If the fibrinolytic system is overall less active in vitally exhausted individuals, one would expect PAI activity to be higher and t-PA antigen level lower in this group. The present study revealed that the PAI activity level was indeed higher in the chronically stressed group, but t-PA antigen did not differ between the groups. PAI and t-PA are for the most part generated in the endothelial cells. If the present study had revealed that both measures of the fibrinolytic system were significantly elevated in the chronically stressed group, a dysfunctioning of the endothelial cells caused by subclinical atherosclerosis could have accounted for such a difference between the exhausted and not exhausted subjects (Ridker et al., 1993). However, in this respect, subclinical atherosclerosis is not a likely explanation for the observed difference between chronically stressed and non-stressed subjects because the groups differed only PAI activity and not on the level of t-PA antigen.

The presence and cause of psychological stress was extensively evaluated in each subject. For allocation to the exhausted group, the symptoms had to be the result of a failure to cope with prolonged psychological stress. Therefore, it was required that there was an increase in one of the three characteristics of exhaustion: excessive tiredness, irritability, or demoralization, during the last year before examination. Patients were excluded if exhaustion was a consequence of a somatic or psychiatric disorder, such as major depression. In other words, the chronically stressed group had to experience feelings of exhaustion as a normal reaction reflecting a breakdown in adaptation to prolonged psychological stress. The results of this study suggest that the relationship between prolonged psychological stress and myocardial infarction may be mediated in part by an imbalance between clotting tendency and fibrinolysis. 



\section{Chapter 7.}

\section{Psychobiological mechanisms of the relationship between vital exhaustion and acute coronary syndromes.}

The principal finding of the present study is that feelings of exhaustion are of predictive value in the clinical course after coronary angioplasty. Furthermore, this association is independent of the severity of underlying coronary artery disease. This result supports the previously reported observations of the Rotterdam Civil Servant Study (Appels \& Mulder, 1988) and the Kaunas Rotterdam Intervention Study (Appels \& Otten, 1992) where it was found that feelings of exhaustion predict future myocardial infarction in apparently heathy individuals.

The relationship between vital exhaustion and future cardiac events raises questions as to the responsible psychobiological mechanism; this is the subject of the present chapter. The first two sections deal with the pathogenesis of spontaneous atherosclerosis and restenosis after Percutaneous Transluminal Coronary Angioplasty (PTCA). Next, it is discussed how progressive coronary disease is related to the development of anginal complaints and myocardial infarction. This chapter continues with a proposed psychobiological model that can explain the relationship between vital exhaustion and future cardiac events. Finally a few suggestions are given for the addition of immunological factors that could be addressed in future studies in the area of cardiovascular behavioral medicine.

\subsection{The pathogenesis of coronary lesions.}

Presently, the model of "response to injury" as formulated by Ross (1986) has received much attention and scientific support. This model combines the hypotheses that atherosclerotic plaques develop from a) increased fibrin deposition in the vessel wall., with secondary lipid accumulation; and b) increased blood-lipid accumulation in the vessel wall, as a result of an imbalance between deposition and removal of bloodlipids. This model is alluded to by Fuster and colleagues (1992) in order to formulate a pathophysiological classification of vascular injury in which three types of lesions are distinguished. Each type of lesion reflects a stage of increasing coronary artery disease on the basis of the extent of damage to the arterial wall. Type I lesions consist of functional alterations of the endothelial cells without substantial morphological changes in the arterial wall; Type II lesions include damage to the endothelium (denudation) and intima while the internal elastic lamina is still intact; and Type III lesions contain endothelial denudation with damage to both the intima and media (figure 1). 


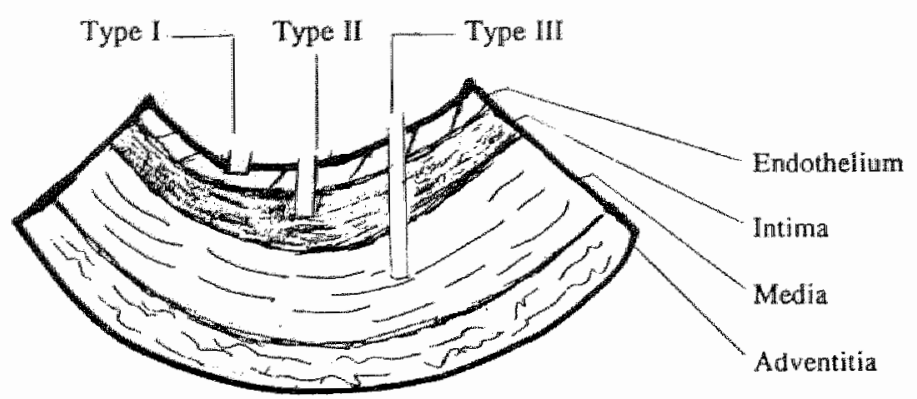

figure 1, Components of the arterial wall and types of lesions that indicate the severity of arterial disease.

Type I injuries often occur at locations where the blood-flow is irregular, especially at bending points, branching sites, or bifurcations. Mild endothelial damage is promoted by several factors, among which hypercholesterolemia, circulating vaso-active amines, infections, and chemical irritants of tobacco smoke. Type I injury leads to the accumulation of lipids and macrophages in the vascular wall.

Platelets, endothelial cells, and macrophages may secrete several growth factors that initiate moving of smooth muscle cells to other layers of the vessell wall (migration) and growth (proliferation) of these cells. This effect on the smooth muscle cells may result in a Type II lesion, that may reflect either deposition of fibrin into the intima (= fibrointimal lesion) or, less frequently, the development of a lipid-lladen lesion (foam cell). This lipid lesion is enclosed by a capsule-like layer of smooth muscle cells and collagen (fibrous cap). In addition, platelets may adhere to these sites.

A Type II lipid lesion can easily be disrupted because of the thin layer of the lesion. This risk is especially present at sites of arterial branching or bending because of the lack of an underlying stable collagen support of the lesion. If a Type II lesion disrupts, clot (thrombus) formation may occur resulting in a Type III lesion. There are two subsequent processes that are involved in the progression from Type II to Type III lesions: 1) platelet adhesion and aggregation; and 2) the formation of fibrin fibers which is activated by thrombin (see also section 2.2.).

The von Willebrand factor is necessary for the adhesion of platelets to the vascular wall. Platelets may activate several growth factors that cause the smooth muscle cells to proliferate and, in second instance, to migrate from the medial vascular layer to the intima.

Following the formation of the initial platelet clot an extra-cellular matrix may develop which includes fibrotic organization of the clot. If the thrombus is small, then this will promote a gradual local increase of the atherosclerotic plaque. On the other 
hand, if the thrombus is large and occlusive, they can contribute to acute coronary syndromes (i.e., unstable angina, myocardial infarction, or sudden cardiac death; see also the next section).

The ultimate role of thrombin is the activation of the transition of fibrinogen into fibrin. But, thrombin also effects the initial phases of the platelet clot formation. Following Type I damage, thrombin (factor $\Pi_{2}$ ) is embodied into the lesion. In second instance it is released as a response to fibrinolysis of the initial clot and also as a result of organization of the clot. Furthermore, thrombin may activate growth related signals in the smooth muscle cells, which is of importance to the initial proliferation of smooth muscle cells as well as the formation of the organized thrombus in the intima.

In sum, atherosclerosis progresses from minor endothelial injury (Type I lesions) via intimal damage (Type II lesions) to thrombus-formation (Type III lesions). Initially platelet adhesion and aggregation as well as smooth muscle cell proliferation play a crucial role. In later stages of coronary artery disease thrombosis and fibrinolysis contribute to the development of Type III lesions.

\subsection{Mechanisms of restenosis after coronary angioplasty.}

The development of new cardiac events after PTCA is often the result of restenosis of the dilated lesion. The incidence of restenosis after successful PTCA varies from 20 $30 \%$, depending on the definition of restenosis, the clinical characteristics of the patients, and the anatomy of the dilated lesion (chapter 5). The process of restenosis differs from spontaneous atherosclerosis in the sense that PTCA induces a Type II (or sometimes Type III) lesion. This occurs because during PTCA, the coronary lesion is not removed but more or less pressed into the intima of the arterial wall (chapter 2). The damage that results usually affects the endothelial and smooth muscle cells and this is thus equivalent to the Type II lesion. In case of a larger dissection of the artery, it may occur that a more severe lesion is created, resulting in acute thrombus formation (Type III lesion) requiring short term re-dilatation. One of the typical characteristics of restenosis following successful PTCA is that it predominantly occurs between two and three months after the initially successful treatment.

Two processes that most probably promote restenosis are early platelet adhesion and aggregation and late intimal proliferation. Thrombus formation at the site of balloon dilatation has been suggested as a pathological mechanism (Chesebro et al., 1987).

In animal studies where the endothelial cells are denudated and the intima is injured (Type II lesion), local platelet adhesion and aggregation occurs which results in the secretion of the glycoprotein platelet derived growth factor (PDGF) and other growth factors. Several findings are in support of the contribution of platelet activity in the initial stages of restenosis: 1) The presence of platelet factor 4 (a constituent of the platelet $\alpha$ granules) within half an hour after balloon injury. Liu et al. (1989) argue that PDGF and other growth factors probably function locally at the site of platelet 
adhesion and that aggregated platelets hardly contribute to the healing process. 2) PDGF may both attract smooth muscle cells from the media to the intima (migration) as well as promole growth of the intimal cells (proliferation). PDGF and other growth factors may also be secreted by endothelial cells, smooth muscle cells, and macrophages. 3) endothelial cells secrete heparin after mild damage but loose this protective capacity after extensive denudation. 4) It appears that the number of smooth muscle cells increases from day 2 to day 7 after balloon injury (Clowes et al., 1985).

However, depletion of platelets does not prevent smooth muscle cell proliferation and platelet inhibition therapy does not reduce the incidence of restenosis. It appears, therefore, that platelet deposition plays a role in early restenosis but that it can not account for the typical late coronary restenosis. Apart form the problem of the delayed onset of restenosis, Liu et al. (1989) additionally refer to the absence of thrombus material in restenosed lesions at autopsy studies. The evidence for excessive intimal thickening (hyperplasia) is more consistent (e.g., Austin et al., 1985). Normally, smooth muscle cells proliferate at a slow rate $( \pm 0.1 \%$ per day). This is probably due to the growth limiting effect of heparin. Endothelial cells, platelets, and macrophages may act as mitogenic stimuli accelerating the growth of smooth muscle cells. For as yet unknown reasons, it appears that some smooth muscle cells are more prone to proliferate than others. After two weeks, intimal thickening is not related to a further increase in the number of smooth muscle cells, but more to an increase in cell volume and the formation of an extracellular matrix and connective fibrotic tissue. The severity of the lesion is an important determinant of intimal proliferation. If the endothelial cells are damaged only, the effect of exposure to platelets is most often not strong enough to initiate intimal thickening. It appears that direct injury to the smooth muscle cells is necessary to induce intimal proliferation (Liu et al., 1989).

In the study described in chapter 5 , the extent of coronary disease was taken into account in order to control for confounding due to underlying coronary artery disease. Three indicators were used: the number of diseased vessels before PTCA and the number of diseased vessels after successful PTCA as well as the severity of residual luminal narrowing. This is a rather global way to evaluate the influence of coronary disease on restenosis. However, more precise measures would not have solved the objection that probable predictors of restenosis were not adequately evaluated. For example, if the minimal luminal diameter of the residual stenosis is considered (e.g., Serruys et al., 1988), then it is not clear what condition preceded the final result of the angioplasty. It might have been that a large "Type II lesion was necessary in order to dilate the artery, with an associated over-stretching of the original vessel wall. Alternatively, the same residual stenosis might have been resulted from a less drastic lesion because the dilatation was easier to perform or because part of the lesion was washed away by the circulation. Because it was not possible to take these phenomena into account, the global measure of coronary artery disease was used. This procedure appears to be adequate the more so because it predicted future cardiac events better than location of the dilatation or the severity of the residual stenosis. 
Future studies on restenosis after PTCA might reveal more insight into the progression of coronary artery disease by taking coagulation and fibrinolysis, as well as growth mediating factors into account. In addition, the echo-Doppler techniques that allow local investigation of the coronary lesion might provide useful insight into the importance of specific lesion characteristics on restenosis of dillated lesions.

\subsection{Factors that contribute to development of acute coronary syndromes.}

In this section, the characteristics of the lesions will be described that lead to reduced coronary perfusion and hence may result in anginal complaints or myocardial infarction. The two processes that mainly account for the reduction of coronary blood supply are the formation of a stabilized the blood-clot and vasoconstriction. It is believed that recurrent episodes of disruption of atherosclerotic plaques, which results in intraluminal thrombosis, leads gradually to coronary occlusion. This process is dynamic and repetitive. Plaque disruption may cause intermittent or transient vessel occlusion and ischaemia by a labile thrombus in some patients with unstable angina. In other patients more severe vascular damage may develop taking the form of a large ulcer. This leads to the formation of a fixed thrombus and a more persistent occlusion, which may result in an acute myocardial infarction. The definite occlusion of a coronary vessel is often caused by a (partial) detachment of a thrombus that lies over a fissured atherosclerotic plaque. On the basis of observations by Falk and colleagues, Fuster et al. (1992) conclude that vascular occlusion is not preceded by one single thrombotic event, but that intermittent thrombus formation and degradation occurs prior to coronary occlusion.

Since Type I and II lesions do commonly not have clinical consequences, the present section is limited to factors that affect Type III lesions. As alluded to above, mild Type III lesions induce adhesion of platelets and white blood cells to the vessel wall which forms an unstable thrombus. During this process several substances are secreted by the platelets, of which thromboxane $A_{2}$ and serotonin are the most important. These substances promote platelet aggregation, vasoconstriction, and intimal proliferation. These three consequences lead to a temporary coronary stenosis, often reflected by unstable anginal complaints. On the other hand, if the Type III lesion is deep, then the thrombogenic component becomes more active, leading to the exposure of collagen and tissue factor to the blood. This results in a stable blood-clot and subsequent myocardial infarction. When a thrombus is formed, fibrinolytic activity may partially dissolve the thrombus. But in the setting of an underlying Type III lesion previously covered collagen and fibrin-bound thrombin is exposed to the blood; this is highly thrombogenic and generally leads to unstable angina or myocardial infarction. The fact that lesion progression is characterized by intermitting thrombosis and fibrinolysis (see below) adds up to this phenomenon.

As for the systemic component of a hypercoagulable state, the circulating catacholamines (adrenaline and noradrenaline) play a contributing role. Catecholamines 
activate platelets and generate thrombin. "This mechanism and catecholamine-dependent vasoconstriction may be of major importance in humans because they may link emotional stress, circadian variation, catecholamine effects, and the development of arteriall thrombosis and vasoconstriction" (Fuster et al., 1992).

Several studies supported the importance of the fibrinolytic system in the development of unstable angina and myocardial infarction in patients with coronary disease (Aznar et al., 1988; Hansten et al., 1987; Olofson et al., 1989) However, there are studies that do not show a prospective relationship between fibrinolytic capacity and future manifestation of coronary disease (Hunt et al., 1990; Oseroff et al., 1990). It has also been argued that the risk associated with a deficient fibrinolytic system merely reflects the presence of subclinical coronary artery disease (Ridker et al., 1993). Other hemostatic proteins that coniribute to thrombus formation are fibrinogen and factor VII. For a more elaborate discussion of the clotting cascade and the fibrinolytic system the reader is referred to chapter 2 , section 2).

In addition to blood coagulation and fibrinolysis, coronary flow is also significantly influenced by factors that promote vasoconstriction. Mild endothelial damage may cause inappropriate vasoconstriction by smooth muscle cell activity after exposure to shear stress, acetylcholine, and bradykinin. Alternatively, intact endothelium can release mediators (endothelial relaxing factor (EDRF) and prostacyclin) that relax the smooth muscle cells and thence dilate the coronary artery. EDRF is responsive to shear stress, acetylcholine, bradykinin, angiotensin II, histamine, ADP, ATP, and noradrenaline. Endothelial cells also contain factors that promote vasoconstriction. For example, endothilin-I induces constriction after stimulation by noradrenaline. In addition, endothilin-I increases smooth muscle cell proliferation. Zeiher and colleagues (1991) postulate that there is an increasing impairment of the endothelium in its vasoactive function, where local endothelial dysfunction characterizes the initial stage of atherosclerosis (Type I lesions), which develops to inappropriate vasoconstriction in over time.

When the arterial lesion is thus severe that the endothelial layer is no longer present, then activated platelets secrete thromboxane $\mathrm{A}_{2}$, serotonin, and adenosine nucleoids (ADP and ATP). This condition causes vasoconstriction. In peripheral arteries this is a functional response that prevents the organism from excessive bleeding. But, in the coronary arteries this response is evidently undesirable. However, when the activated platelets reach the intact endothelium that is located more distally, then vasodilatation occurs at that distal site. The latter effect is the result of EDRF on intact endothelium and may prevent severe ischemia of the whole traject.

In sum, the disruption of small plaques is of importance to the pathogenesis of acute myocardial infarction, whereas prolonged severe stenoses more commonly result in gradual vessel occlusion. In the latter situation a small or silent infarction may occur, or no infarction at all which might be the beneficial result of well-developed collateral blood supply to the distal portion of the stenosed vessel. In this respect it is of relevance to note that Webster and colleagues (1990) observed in a five year follow-up 
study that coronary lesions of Type III progress three times as often to total occlusion than less severe lesions (Type $I$ and II). Of interest is that the infarct related lesions were initially of hemodynamically minor importance $(<75 \%$ stenosis). This is congruent with the observation of Ambrose et al. (1988) who showed that in more than two thirds of patients with myocardial infarction, the previously observed coronary stenosis was less than $70 \%$. The formation and removal of stabilized blood clots as well as the factors that induce vasoconstriction determine for the most part coronary blood flow. There are a number of lesion specific and systemic factors that promote clot stabilization and vasoconstriction. The next section will discuss how these factors might be affected by vital exhaustion.

\subsection{The relationship between vital exhaustion and the biological mechanisms of acute coronary syndromes.}

The overall hypothesis that will be formulated in the present section is that vital exhaustion is related to the occurrence of acute cardiac events. We will discuss the consequences of exhaustion in the setting of the types of lesions as described in the previous sections. The proposed psychobiological mechanism is that vital exhaustion results in an imbalance between coagulation and fibrinolytic activity which destines for acute coronary syndromes.

The development of Type I lesions is, among other factors, promoted by hypercholesterolemia and vasoactive amines like adrenaline and noradrenaline. The negative consequences of real life stress on blood lipid levels was demonstrated in several studies (e.g., Friedman et al., 1958; Kasl et al., 1968; Taggart \& Carruthers, 1971). In the study by Kasl et al (1968) it was observed that loss of work was related to higher serum cholesterol levels. Van Doornen (1988) investigated a real life stressor in healthy male subjects (the defence of their doctoral thesis). A significant correlation was observed between the level of exhaustion and the increase in cholesterol during exposure to this stressor. Van Doornen (1988) also found a positive association between exhaustion and increase in adrenaline during stress. These data indicate that exhausted persons may have higher levels of blood lipids and catecholamines in response to psychological stressors. Consequently, this may contribute to the development of Type I lesions in these individuals.

Until now, no information is available as to the relationship between objectified Type I and Type II lesions and vital exhaustion. Animal studies have shown that the central nervous system is involved in the pathogenesis of Type I and Type II lesions. Electrical stimulation of the lateral hypothalamus in conscious unrestrained animals on normal diets induces severe endothelial damage and denudation in the coronary arteries. If the stimulation is continued, then intimal lesions develop with features of atherosclerotic plaques (Gutstein, 1988). Given the endothelial dysfunction in these lesions, it is of importance to note that coronary ischemia may result from inappropriate vasoconstriction after physical and/or psychological stressors (Yeung et al., 1989; 
1991). This leads to the intriguing hypothesis that vital exhaustion is related to the presence of endothelial dysfunction. However, as was alluded to above, the mere presence of endothelial dysfunction is not a sufficient condition for the progression of thrombogenic coronary artery disease nor for the development of acute coronary syndromes. For these events to occur, more severe lesions have to be present that also affect the smooth muscle cells.

As far as the relationship between vital exhaustion and more severe coronary artery disease (Type III lesions) is concerned, it was shown in chapter 3 that there was no substantial association between the extent of coronary artery disease and vital exhaustion. This could have partially been caused by the heterogeneity of the group of consecutive patients referred for diagnostic angiography in the setting of symptoms of myocardial ischemia. The association between extent of coronary artery disease and vital exhaustion gained strength when the analysis was restricted to those patients who received PTCA (chapter 4). But also in the latter instance, the explained variance of the exhaustion scores on the Maastricht Questionnaire could hardly be accounted for by the severity of coronary artery disease (less than 5\%). As mentioned in chapters 3 and 4 , this indicates that the relationship between vital exhaustion and future cardiac events as observed by Appels and colleagues $(1988 ; 1992)$ is unlikely to be confounded by underlying coronary artery disease. Moreover, unstable angina and myocardial infarction usually develop from Type III lesions that are relatively mild (i.e., leading to a coronary stenosis of less than $75 \%$ ). This is also in line with the idea that the association between vital exhaustion and acute coronary syndromes is probably mediated by another pathway than gradual progressive coronary artery disease.

The initial stages of acute occlusion of coronary vessells are characterized by platelet adhesion to the (fissured) arterial wall. Lulofs (1990) compared the platelet activity of exhausted and non-exhausted subjects during rest and during a laboratory stress task. Platelet aggregation was measured making use of the methods developed by Brown and the in vivo platelet activity assessment of $W_{\mathrm{u}}$ and Hoaz, respectively. The results revealed no differences between exhausted and non-exhausted persons. With respect to the development of acute cardiac events, these investigations do not suggest an important contribution of the initial platelet plug formation in exhausted subjects. As was mentioned in the previous section, the pathogenesis of late restenosis after successful PTCA is more dependent on hyperplasia than on platelet adhesion and aggregation. This may point to the importance of growth and migration promoting mediators as an explanatory pathway for the relationship between vital exhaustion and new cardiac events after successful PTCA (see also section 7.5).

The processes that are involved in the formation of a stabilized blood clot (coagulation and fibrinolysis) were investigated in the study described in chapter 6. It was shown that vital exhaustion was associated with a reduced fibrinolytic capacity. More precisely, exhausted subjects were characterized by increased levels of plasminogen activator inhibitor. Because acute coronary syndromes occur following intermittent thrombus formation and degradation prior to coronary occlusion (section 7.3.), this 
reduced fibrinolytic capacity is likely to be of major importance to the relationship between vital exhaustion and acute coronary syndromes, especially myocardial infarction.

Vital exhaustion is conceived as an end stage of prolonged psychological stress (see chapter 1, section 3). There are individuals who, as a consequence of their personality, experience more stressful events than others. Hostile (or Type A) individuals are more prone to encounter distressing situations and are also likely to cope with these circumstances in a more demanding way. This has lead to the assumption of an interaction between vital exhaustion and hostility as related to future cardiac events. Previous case-control studies have given support for this hypothesis (chapter 1, section 2.2.). Personality characteristics such as hostility or Type A behavior are characterized by prolonged activation of the sympathetic nervous system. This promotes (gradual) progression of coronary artery disease. Excellent reviews on this topic have been given by Kaplan et al. (1991) and Williams et al. (1991). Overall, there appears to be a consistent relationship between measures of hostility and the severity of coronary artery disease as objectified during coronary angiography. On the other hand, the contribution of exhaustion to the gradual progression of coronary atherosclerosis appears to be of minor importance because the relationship between vital exhaustion and the severity of coronary artery disease was shown to be very weak (see chapters 3 and 4 ). The influence of exhaustion on the development of acute coronary symptoms may be related to an impaired balance between thrombus formation and fibrinolytic activity in exhausted individuals (see also, Pietraszek MH et al., 199l). It is likely that this imbalance has more severe consequences when atherosclerosis has progressed to the stage of Type III lesions. This may also explain the interaction between vital exhaustion and hostility as associated with future cardiac events. Hostility sets the stage for acute coronary events by promoting gradual progression of atherosclerosis and in addition to this, vital exhaustion contributes to the oecurrence of acute coronary blockage by its incapacitating effect on the fibrinolytic system. Figure 2 shows the hypothesized model. It incorporates the contribution of 1 ) the progression of coronary disease over time using the topology of Fuster et al. (1992); 2) age; 3) hostility; and 4) vital exhaustion.

Figure 2 shows that the progression of coronary artery disease is faster in hostile persons. In addition, the dashed lines indicate the time at which exhaustion occurs. The periods of exhaustion are supposed to be associated with a reduction of the fibrinolytic capacity which results in an increased proneness for acute coronary syndromes due to the tendency to form stabilized blood-clots. This does not result in acute coronary syndromes as long as the coronary artery disease has not progressed to substantial Type II lesions. In that setting, and even more so in the setting of Type III lesions, the impaired fibrinolytic capacity substantially increases the risk of acute coronary events. When the process of gradual progressive atherosclerosis persists, collateral vessels may develop which decreases the risk of acute coronary events in the long run. Because coronary disease develops faster in hostile persons, the decrease 
due to this development of collaterals is also observed at an earlier time. This may explain why the relationship between hostility and atherosclerosis is stronger below the age of 55 years. Another important aspect of figure 2 is that vital exhaustion may be a recurrent phenomenon, especially in hostile persons. This is congruent with the observation that exhausted individuals often report previous periods of being "overstressed ${ }^{*}$ or burned out. In addition, the model predicts that the interaction between vital exhaustion and hostility is the strongest at younger ages (40 to 50 years).

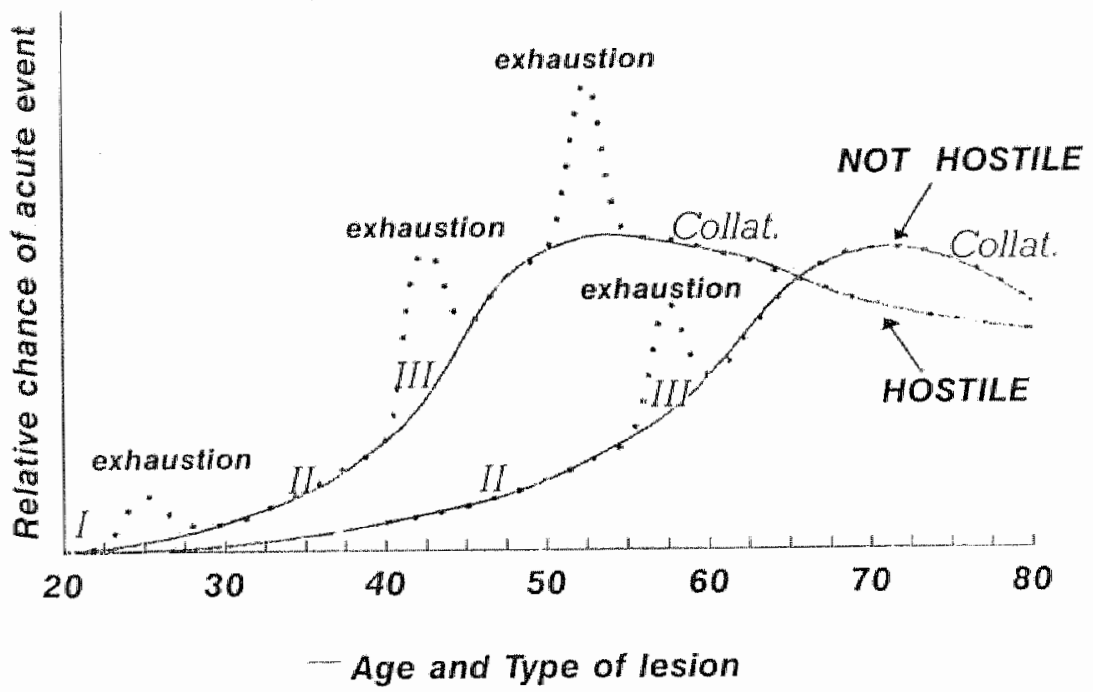

Figure 7.2. A model that integrates the multiplicative effect of vital exhaustion and hostility on progressive coronary artery disease and subsequent acute coronary syndromes. Two graphs are presented; one solid line shows the progression of cononary artery disease in hostile persons which peaks at the age of 53 , and a second solid line that shows the progression of coronary disease in persons that are not hostile with the peak at 70 years. On the solid lines the severity of the coronary lesion is indicated with Romam figures as well as the onset of the development of collaterals with its contingent decrease in the risk of acuite events. The dashed lines indicate episodes of vital exhaustion which are shown to occur more often in hostile persons than in those who are not hostile. The figure depicts that episodes of exhaustion are associated with an increased risk of acute coronary events, especially when type III lesions are present.

\subsection{The relationship between vital exhaustion and immunological aspects of atherosclerosis.}

Apart from the contribution of thrombogenic factors and fibrinolysis in the development of acute coronary syndromes there may also be other biological contributors of the progression of coronary artery disease, namely immunological and growth mediating factors. During the last decade new evidence has accumulated which 
indicates that the human atherosclerotic plaque is more than a static accumulation of lipids in the arterial wall. Coronary lesions contain living cells which thave a variety of specialized regulatory functions that influence the progression of atherosclerosis. Advances in monoclonal antibody as well as DNA technology have gained insight into the different types and functions of endothelial cells and smooth muscle cells of the arterial wall. In animals with a lipid-rich diet, atherosclerotic lesions consist predominantly of macrophages ( $=$ mononuclear phagocytes). The present section deals with the possible relationship between prolonged psychological stress, impaired immune function, and the accelerated progression of coronary artery disease. In order to understand the impact of the immune system on the atherosclerotic process, a short outline of this system is provided.

The immune system consists of an aspecific and a specific component. The aspecific immune system prevents foreign elements (antigens) to penetrate the organism. It includes the peristaltic system, bowels, and the skin, among others. The specific immune system consists of the humoral immunity (B-cells) and cellular immunity (Tcells). When a foreign element penetrates the blood circulation, it is recognized and processed by a macrophage. The macrophage presents the antigen to a $\mathrm{B}$ or $\mathrm{T}$ lymphocyte. B-cells react by producing antibodies (immunoglobulins; IgA, IgE, IgG, IgM, etc.) which bind to the foreign element (antigen). These complexes of antibodyantigen are then eliminated from the circulation by activated complement proteins. If an antigen is presented to a $\mathrm{T}$-cell several reactions can occur. The $\mathrm{T}$-cell may directly eliminate the antigen (cytotoxic T-cells). In other circumstances the T-cells initiate the humoral immune system, by $\mathrm{T}$-helper and $\mathrm{T}$-suppressor cells. After penetration of an antigen, $T$-memory cells are generated to enhance the effectiveness of the immune reactivity in case of future infections. Another component of the immune system is the natural killer cell, which can directly destroy virus-affected cells as well as tumor cells. Insufficient immunity may result in infectious diseases (flu, mononucleosis, measles etc.). Alternatively, allergies reflect an over-reactive immune response to an antigen.

T-cells are found in atherosclerotic lesions. Libby and Hansson (1991) argue that the magnitude of the inflammatory component is often underestimated because of the dependency of the monoclonal antibody recognition of cell-types on the physiological state of the cell. B-cells and natural killer cells are not prevalent in the atheroselerotic plaque.

T-cells may be passively deposited in the developing atherosclerotic plaque, but are more of relevance if they are activated immunologically. T-cell activity at smooth muscle cells and endothelial cells is marked by gamma-interferon (IFN- $\gamma$; also known as lymphokine immune interferon, or immune interferon). IFN- $\gamma$ is a cytokine that can be secreted by natural killer cells and T-cells. Since natural killer cells are virtually absent in the atherosclerotic plaque, IFN- $\gamma$ reflects the activity of T-cells. Additional evidence for T-cell activity comes from the observation that T-cells in atherosclerotic plaques exhibit interleukin-2 (IL-2) receptors. These indicators of activated T-cells are 
also observed at later stages of atherosclerosis.

For the T-cell to become active, there must be antigenic stimulation, that is, a foreign surface that activates the immune system. In atherogenesis, possible candidates are: oxidized lipoproteins (especially apolipoprotein-B fragments), antigens exposed to blood due to necrosis, virall infection (herpes-types and cytomegalovirus). Activation of $T$ (and B) lymphocytes is promoted by several cytokines of which the interleukins are studied most extensively. Originally it was thought that $\mathrm{IL}$ was only present in leukocytes, but it is now clear that smooth muscle cells and endothelial cells contain IL- 1 and IL-6. IL-6 contributes most to the stimulation of the T and B-cell activity. IL-1 appears to be more important in the cell-cell contact (Libby \& Hansson, 1991). IL-8 may both promote and inhibit white blood cell accumulation in the atherosclerotic plaque. In addition, tumor necrosis factor can be produced by smooth muscle cells and may contribute to atherosclerosis progression as well.

Blood-cells in the vessel wall do not only express immuno-active substances, but may also react to immunollogical stimulation. If the vessel wall is damaged, then the endothelium will become active. This leads to adhesion and migration of leukocytes and monocytes, which will be followed by secretion of growth factors and cytokines. The result of these processes is that smooth muscle cells migrate and peroxidated lipids attach to the arterial wall (Van Dam-Miras et al., 1992; Vossen, 1993). IL-1 and tumor necrosis factor stimulate the adhesion of leukocytes to the endothelium. These two cytokines also regulate the interaction between the endothelium and the platelet adhesion, as well. as clotting and fibrinolytic factors (Stern et all., 1985; Bevilacqua et al., 1984; Bevilacqua et al., 1986; Nachman et al., 1986) promoting stabilized clots. In addition to leucocyte adhesion to the endothelium, the white blood cells may also penetrate the endothelium in new lesions. This migration is probably activated by cytokines (IFN- $\gamma, \mathrm{IL}-1, \mathrm{IL}-6, \mathrm{IL}-8$, and tumor necrosis factor), oxidized lipoproteins, and chemotactic complement activation product. This effect on hemostasis and thrombosis probably contributes to the transition from chronic to acute coronary artery disease (Libby \& Hansson, 1991).

In a study by Hansson and colleagues the importance of the immune-system for the progression of atherosclerosis is clearly demonstrated. Three types of rats were used for this study. In one group the T-cells were eliminated by a monoclonal antibody; a second group consisted of rats where no $T$ lymphocytes were present because of removal of the thymus; and a third group was used as a control group. The growth of the intima was investigated after experimentally induced damage to the wall of the common carotid artery. The animals that were $T$-cell deficient showed increased atherosclerotic plaques. In addition, Hansson et al. showed that infusion with IFN- $\gamma$ resulted in a significantly reduced intimal thickening after vascular damage. The study supports the theory that T-cells inhibit progression of atherosclerosis and that this mechanism is activated by IFN- $\gamma$.

In the initial stages of atherosclerosis (Type I) the lesions are composed of layers of macrophage derived foam cells. These macrophageous foam cells probably consist of 
blood monocytes that have penetrated the vascular wall, lipid-laden smooth muscle cells, and also extracellular lipids. As mentioned above, these lesions most often become fibromuscular, but may also develop to fibrolipid lesions that are prone to disrupt. Macrophages play multiple functions in the progression of coronary artery disease. Although the picture is far from complete at present, it has been convincingly documented that: 1) Macrophages influence the uptake and metabolism of lipids; 2) Macrophages promote the transportation and oxidation of low density lipoprotein (LDL) cholesterol; 3) Macrophages contribute to the release of several growth factors (= inducing smooth muscle cell proliferation); 4) Macrophages may generate toxic products (products of lipid oxidation; free radicals) that increase atherosclerosis leading to intimal damage (Type II lesions); 5) Macrophages may produce proteases ( = enzymes that dissolve proteins or peptides) which destruct the organized extracellular matrix to form lesions that are much more prone to disrupt ultimately leading to Type III lesions. In addition, 6) Macrophages can promote the thrombus formation by secreting tissue factor and hence plasminogen activator inhibitor (Libby \& Hansson, 1991).

In sum, the presence of immuno-active substances in the onset and progression of atherosclerosis is supported by several investigations. It remains to be shown, however, if this activity is secondary to atheroscleroses reflecting a reaction to antigenic stimulation, or whether immune functions play a primary role in the onset and development of atherosclerosis. This issue is of essential relevance for the development of lesions after transplantation and coronary angioplasty because in these conditions underlying coronary lesions already exist. This may result in differences from spontaneous atherosclerosis on initially normal vessels as described above.

The impact of immunological processes on restenosis after PTCA was recently suggested by Libby and colleagues (1992). Although platelets do not relate to the growth of smooth muscle cells, they contribute to the migration of these cells to the intimal vascular layer. In other words, activated platelets may be more important as chemoattractants of smooth muscle cells than as mitogens. It is very likely that balloon injury in lesions consisting of numerous macrophages (as is the case in PTCA) is essentially different from the effect of balloon injury to normal arteries. In a cascade model of restenosis Libby et al. state that: "acute local thrombosis, blood coagulation, and/or mechanical injury first activate cytokine gene expression by macrophages and/or smooth muscle cells within the plaque. This acute cytokine expression would evoke secondary, self-sustaining, and continuing autocrine and paracrine growth factor and cytokine expression by lesional cells including leukocytes that could account for the lag between injury and restenosis and failure of chronic antithrombotic therapy to retard this process". Of relevance to restenosis is that $\mathrm{IL}-1$ can stimulate PDGF and other growth mediators by way of autoinduced accentuation. As mentioned above, IL1 is generally not present in unstimulated vascular cells. But IL-I can be activated for example by bacterial endotoxin. Moreover, IL-1 and tumor necrosis factor levels showed a stronger response to bacterial endotoxin in atherosclerotic rabbit arteries 
than in normal arteries. Loppnow and Libby (1992) found a low basal level of IL-1 in isolated human atherosclerotic plaques, which was markedly increased after exposure to endotoxin. This indicates that the immune system is likely to be involved in progression of coronary disease after coronary angioplasty.

There appears to be an interaction between coagulation, fibrinolysis, and immunoactivity (see above) and hence the contribution of the immune system seems to be a promising area of research in cardiovascular behavioral medicine. It has been shown that acute experimental as well as prolonged real life stressors reduce the efficacy of the immune system (Brosschot et al., 1991; Glaser et al., 1986; Jemmott et al., 1984; Zakowski et al., 1992).

No investigations have been performed that relate immune functions to vital exhaustion. In the study reported in Chapter 6, no association was found between segmentation of white blood cells and vital exhaustion. This ruled out that the feelings of exhaustion reflected manifest infectious disease. But more subtle immunological deficiencies may be present. This corresponds to one of the impressions of the casecontrol studies of Falger and Meesters, where a number of coronary patients complained about the nuisance of not fully recovering from influenza during the last months before their myocardial infarction. On the other hand, fatigue may be the consequence of immune deficiency. This is, for example, one of the hypothesized explanations of the Chronic Fatigue Syndrome (see Chapter 1.4.3.). Although the Epstein-Barr virus is not identified unambiguously in this syndrome, a few studies suggest the presence of a deficient immune function (e.g. Hamblin et al., 1983; Tosto et al., 1985; Caligiuri et al., 1987; Strauss et al., 1988). Exhaustion and infectious states are probably mutual reinforcing phenomena. In addition, it is observed that quite a few $\mathrm{T}$ lymphocytes are found in atherosclerotic lesions (Emeson \& Robertson, 1988; Gown et al., 1986; Libby \& Hansson, 1991; Jonasson et al., 1988). It may be that a reduced fibrinolytic capacity in combination with a dysfunctioning of the immune system explains the predictive value of vital exhaustion for future cardiac events after coronary angioplasty.

Psychophysiological theories often address the consequences of hyperractivity in order to explain the accelerated development of disease. However, it is of importance to note that hyperactivity may turn into hyporeactivity after continuous exposure to stress. Future research in this area is probably most fruitful if it is directed to the question which adaptive mechanisms to psychological stressors are diminished or lost when psychological stress persists and leads to a state of exhaustion. The present study shows that vital exhaustion is of predictive value for new cardiac events after coronary angioplasty and that exhausted individuals have an increased tendency to stabilize blood clots. This may be superimposed on progressive atherosclerotic disease which might be related to certain personality characteristics such as hostility. The impact of the immune system would be an interesting subject for future research in cardiovascular behavioral medicine, which might be of relevance for the onset of acute coronary syndromes as well as restenosis after coronary angioplasty. 


\section{References.}

Alonzo A, Simon A, Feinleib M. Prodromata of myocardial infarction and sudden death. Circulation 1975; 52 : 1056-1062.

Ambrose JA, Tannenbaum NA, Alexopoulos D, et al. Angiogiaphic progression of coronary artery disease and the development of myocardial infarction. Am J Coll Cardiol 1988; 12: 56-62.

Anda R, Williamson D, Jones D, Macera C, Eaker E, Glasman A, Marks J. Depressed affect, hopelessness, and the risk of ischemic heart disease in a cohort of U.S. adults. Epidemiology 1993; 4 285-294.

Andreotti F, Davies GI, Maseri A, Kluft C. Fibrinolytic inhibition in blood; circadian fluctuation and possible relevance to coronary artery disease. In: Predisposing conditions for acute ischemic syndromes, Garmisch-Partenkirchen: Steinkopf Verlag Darmstadt, October 14-15 1988, 1-11.

Anzar J, Estellés A。 Tormo $G$ et al. Plasminogen activator inhibitor activity and other fibrinolytic variables in patients with coronary artery disease. Br Heart J 1988; 59: 535-541.

Appels A. Psychollogical prodromata of myocardial infarction and sudden death. Psychoth Psychosom $1980 ; 34: 187-195$.

Appels A. Mental precursors of myocardial infarction. Br J Psychiat 1990; 156: 465-71.

Appels A, Falger PRJ, Schouten E. Vital exhaustion and myocardial infarction in women. J Psychosom Res; in press.

Appels A, Höppener P, Mulder P. A Questionnaire to assess premonitory symptoms of myocardial infarction. Int $J$ Cardiol $1987 ; 17$ : 15-24.

Appels A, Mendes de Leon CF. The association between vital exhaustion, unstable angina, and future myocardial infarction. In: won Arnim Th, Maseri A, eds. Predisposing condition for acute ischemic syndromes. Darmstadt, Germany: Steinkopf Verlag, 1989; 51-57.

Appels A, Mulder P. Excess fatigue as a precursor of myocardial infarction. Eur Heart J 1988; 9 ; $758-764$.

Appels $A_{\text {, Mulder }}$. Fatigue and heart disease. The association between vital exhaustion and past, present and future coronary heart disease. J Psychosom Res 1989; 33: 727-738.

Appels A, Otten F. Exhaustion as precursor of cardiac death. Brit I Clin Psychol 1992; 31: 351-356.

Appels A Schouten E. Burnout as a risk factor for coronary heart disease. J Beh Med 1991; 14. 53. 58.

Appels A Schouten E. Waking up exhausted as risk indicator of myocardial infarction. Am J Cardiol $1991 ; 68: 395-398$.

Ardissino D, Barbers P, De Servi S, et al. Abnormal coronary vasoconstriction as a predictor of restenosis after successful coronary angioplasty in patients with unstable angina pectoris. $N$ Engl $J$ Med 1991; 325: 1053-1057. 
Arlow J. Identification mechanisms in coronary occlusion. J Psychosom Res 1945; 7: 195-209.

Arrindell WA, Ettema JHM. SCL-90; handleiding bij een mulltidimensionele psychopathologieindicator. Lisse: Swets \& Zeitlinger, 1986.

Bar $\mathbb{F} W$, Brugada $P$, Dassen WR, van der Werf $T$, Wellens $H J$. Prognostic value of $Q$ waves, R/S ratio, loss of $\mathrm{R}$ wave woltage, ST-T segment abnormalities, electrical axis, low voltage and notching: correlation of electrocardiogram and left ventriculogram. J Am Coll Cardiol 1984; 4: 17-27.

Bengtson JR, Mark DB, Honan MB, et al.: Detection of restenosis after elective percutaneous transluminal coronary angioplasty using the exercise treadmill test. Am J Card 1990; 65: 28-34.

Bewillacqua MP, Pober JS, Majeau GR, Cotran RS, Gimbrone MA. Interleukin-1 (IL-1) induces biosynthesis and cell surface expression of procoagulant activity in human vascular endothelial cells. J Exp Med 1984; 160: 618-623.

Bevilacqua MP, Schleef R, Gimbrone MA, Loskutoff DJ. Regulation of the fibrinolytic system of cultured human vascular endothelium by $\mathbb{L L}-\mathrm{I}$. J Clin Invest 1986; 78: 587-591.

Booth-Kewley S, Friedman H. Psychological predictors of heart disease: a quantitative review. Psychol Bull 1987; 101; 343-362.

Bouman TK. The measurement of depression with questionnaires. PhD Dissertation: Groningen, 1987.

Brandenburg RO, Fuster V, Giuliani ER, McGoon DC eds. Cardiology: fundamentals and practice. Chicago, London: Yearbook Medical Publishers, 1987.

Braunwald E: Heart disease: 3rd edition. Philadelphia, London, Toronto, Montreal, Sydney, Tokyo: WB Saunders Comp, 1988.

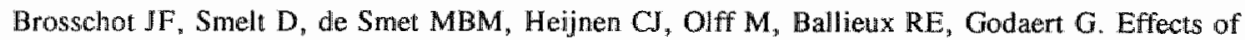
experimental psychological stress on T-lymphocytes and NK cells in man. I Psychophysiol 1991; 5 : $59-67$.

Brozovic M, Stirling Y, Harricks $C_{n}$ North WRS, Meade TW. Factor VIII in an industrial population. Br J Haematol 1974; 28: $381-39$ 1.

Bruce RA. Exercise testing of patients with coronary heart disease, principles and normal standards for evaluation. Ann Clin Res 1971; 3: 323-332.

Bruggeman DA, van Dam-Mieras MCE. The possible role of cytomegalovirus in atherogenesis. Prog Med Virol 38. Melmick JL (ed). Basel: Karger, 1991, 1-26.

Bruhn JG, McCrady KE, Plessis A. Evidence of emotional-drain preceding death from myocardial infarction. Psychiat Digest 1968; 29: 34-40.

Byrne DG. Attributed responsibility for life events in survivors of myocardial infarction. Psychother Psychosom 1980; 33: 7-13. 
Calliguiri M, Murray $C$, Buchwald D, et al. Phenotype and functional deficiency of natural killer cells in patients with chronic fatigue syndrome. J Immunol 1987; 139:3306-3313

Cannon WB, Mendenhall WL. Factors affecting the coagulation time of blood; VI the hastening of coagulation in pain and emotional excitement. Am J Physiol 1914;34: 251-61.

Carney R, Rich M, Freedland K, Saini J, te Velde A Simeone C, Clark K. Major depressive disorder predicts cardiac events in patients with coronary heart disease. Psychosom Med: $1988 ; 50 ; 627-633$.

Cheseboro JH, Lan JY, Fuster V. The pathogenesis and prevention of aortocoronary vein bypass graft occlusion and restenosis after arterial angioplasty; role of vascular injury and platelet thrombus deposition. J Am Coll Cardiol 1986; 8 sub B: 57-66.

Clauss A. Gerinnungsphysiologische Schnellmethode zur Bestimmung des. Fibrinogens. Acta Haemat $1957 ; 17: 237-246$.

Chowes AW, Clowes MM, Fingerle J, Reidy MA. Regulation of smooth muscle cell growth in injured artery. J Cardiovasc Pharmacol 1989; 14 sup: SI2-S15.

Cohen J. Statistical power analysis for the behavioral sciences, 2 nd ed. Hillsdale: L Erlbaum Associates, 1988.

Cohen RJ, Epstein SE, Cohen LS, Dennis LH. Alternations of fibrinolysis and blood coagulation induced by exercise, and the role of beta-adrenergic-receptor stimulation. Lancet 1988; ii: 1264-1266.

Cohn PF. Silent myocardial ischemia and infarction, 2nd edition. New York: Marcel Dekker Inc, 1989.

Costa PT, Zonderman AE, Engel BT, Baile WF, Brimlow BL, Brinker J: The relation of chest pain symptoms to angiographic findings of coronary artery stenosis and neuroticism. Psychosom Med $1985 ; 47: 285-293$.

Crisp A, Queenan M, D'Souza MF. Myocardial infarction and the emotional climate. Lancet 1984; 1 : 616-619.

Croyle RT, Sande GN. Denial and confirmatory search: paradoxical consequences of medical diagnosis. J Appl Social Psychol 1988; 18: 473-490.

David $A_{n}$ Pelosi A, McDonald E et al. Tired, weak, or need of rest; fatigue among general practice attenders. BMJ 1990; 301: 1199-1202.

Denollet J. Emotional distress and fatigue in coronary heart disease, the global mood scale (GMS). Psychol Med 1993; 23: 111-121.

Derogatis LR. SCL-90; administration, scoring, and procedure manual for the revised version. Baltimore, John Hopkins University, 1977. 
Detre K, Hulukov R, Kesley $\mathrm{S}$, et al. Co-investigators of the National Heart, Lung, and Blood Institute"s Percutaneous Transluminal Coronary Angioplasty Registry: One-year follow-up results: National Heart, Lung, and Blood Institute"s Percutaneous Transluminal Coronary Angioplasty Registry. Circulation $1989 ; 80: 421-428$.

van Diest $\mathbf{R}$. Subjective sleep characteristics as coronary risk factors, their association with type A behavior and wital exhaustion. IJ Psychosom Res 1990; 34: 415-426.

vam Diest R, Appels A. Vital exhaustion and depression: a conceptual study. J.Psychosom Res 1991; $35: 535-544$

van Diest R, Appels A. A sleep-physiological study of exhausted and non=exhausted males. Psychosom Med: in press.

Dimsdale $J$, Gilbert $J$, Hutter $A$, Hackett Th, Block P. Predicting cardiac morbidity based on risk factors and coronary angiographic findings. Am J Cardiol 1981; 47: 73-76.

Dixhoorn van J, Duivenvoorde H, Staal J,Pool J and Verhage F (1987) Cardiac events after myocardial infarction: possible effects of relaxation therapy. Eur Heart $\mathfrak{J} 8: 1210-1214$.

Doornen van LJP: Psychological stress reactivity, its relation to behavioral style, mood, sex, and aerobic fitness: Dissertation: Free University Amsterdam, 1988.

Dotter CT, Judkins MP. Transluminal percutaneous treatment of atherosclerotic obstruction; description of a new technic and a preliminary report of its application. Circulation 1964; 30: 654670 .

Emeson EE, Robertson AL. T lymphocytes in aortic and coronary intimas. Am J Pathol 130; 1988: $369-376$.

Falger PRI. Life-span development and myocardial infarction: an epidemiological study. Ph.D. Dissertation Mastricht: 1989.

Falger PRJ, Appels A, Schouten EGW, Verink H. Gender differences in psychosocial precursors of myocardial infarction. Second international congress of behavioral medicine. 1992 (paper: Harnburg, july $15-18)$.

Falger PRI, Schouten E. Exhaustion, psychological stressors in the work environment, and acute myocardial infarction in adult men. J Psychosom Res 1992; 36: 777-786.

Feinleib M, Simon A, Gillum R, Marjolis J. Prodromal symptoms and signs of sudden death. Circulation $1975 ; 51,52$ sup: $155-159$.

Fisher HK, Dun B, Winters WL. Emotional factors in coronary occlusion II; time patterns and factors related to onset. Psychosomaties 1964; 5: 280-291.

Fraser GE. Sudden death in Auckland. Austr NZ I Med 1978; 8: 490-499. 
Frasure-Smith $N$, Prince R: Long-tern follow-up of the ischemic heart disease life stress monitoring program. Psychosom Med 1989; $51: 485-513$.

Freeman L, Nixon P. Time to rethink the clinical syndrome of angina pectoris? Implications for ambulatory ST monitory. Quart J Med 1987; 62: 25-32.

Freudenberger HJ. Staff Burnout. J Soc Issues 1974; 30: 159-165.

Friedman M, Rosenman RH, Carroll V. Changes in serum cholesterol and blood clotting time in men subjected to cyclic variation of occupational stress. Circulation 1958; 17: 852-61.

Friedman M, Thoressen CE, Gill JJ et al.: Alteration of type A behavior and its effects on cardiac recurrences in post myocardial infarction patients. Summery results of the recurrent coronary prevention project. Am Heart J 1986; 112: 653-665.

Fuster $\mathrm{V}$, Badimon $\mathrm{L}$, Badimon J, Cheseboro J. The pathogenesis of coronary artery disease and the acute coronary syndromes. New Eng J Med 1992; 326: 242-250, 310-318.

Gillum R, Feinleib M, Margolis JR, Fabsitz R, Brasch R. The pre-hospital phase of acute myocardial infarction and sudden death. Preventive Med 1976, 5: 408 413.

Glaser R, Rice J, Speicher CE, Stout JC, Kiecolt-Glaser JK. Stress depresses interferon production and natural killer cell activity in humans. Beh Neurosci 100; 1986: 675-680.

Glass DC. Behavior pattern, stress and coronary disease. Hillsdale: Lawrence Erlbaum, 1977.

Gown AM, Tsukada T, Ross R, Human atherosclerosis II, immunocytochemical analysis of the cellular composition of human atherosclerotic lesions. Am J Pathol 125; 1986: 191-207.

Greene WA, Goldstein S, Moss A. Psychosocial aspects of sudden death. Arch Int Med 1972; 129: $725-731$.

Grünzig AR, Senning A, Siegenthaler WE. Non-operative dilatation of coronary artery stenosis. New Engl I Med 1979; $301: 61-68$.

Gunn CG, Hampton JW. CNS influence on plasma levels of factor VIII activity. Am J Physiol 1967 ; 212: $124-130$.

Gutstein WH. The central nervous system and atherogenesis: endothelial injury. Atherosclerosis 1988; 70: 145- 154 .

Hahn P. Der Herzinfarkt in psychosomatischer Sicht. Göttingen; Verlag für medizinische Psychologie in Verlag Van den Hoeck und Ruprecht, 1971.

Haines AP, Imeson JD, Meade T. Phobic anxiety and ischaemic heart disease. Br Med J 1987; 295 : 297-299. 
Hallstrom $T$, Lapidum $L$, Bengtson $C$ et al. Psychosocial factors and risk of ischemic heart disease and death in women. A. 12 year follow-up of participants in the population study of women in Gothenberg, Sweden. J Psychosom Res 1986; 30: 451-459.

Hanblin TJ, Hussian J, Akbar AN, Tang YC, Smith JL, Jones DB. Immunological reasons for chronic ill bealth after infectious mononucleosis. Br Med J 1983; 287: 85-88.

Hamsten $A$, Walldius $G$, Szamosi $A$ et al. Plasminogen activator inhibitor in plasma; risk factor for recurrent myocardial infarction. Lanceet 1987; ii: 3-9.

Hansson GK, Holm J, Holm S, Fotev Z, Hendrich HJ, Fingerle J. T lymphocytes inhibit the vascular response to imjury. Proc Natl Acad Sci USA 1991; 88: 10530-10534.

Hermans WRM, Rensing BJ, Strauss BH, Serruys PW: Prevention of restenosis after percutaneous. transluminal coronary angioplasty; the search for a "magic bullet". Am Heart J 1991; 122: 171-187.

Holmes GP, Kaplan $\mathbb{S}$, Grentz NM et al. Chronic fatigue syndrome; a working case definition. Ann Int Med 108; 1988: 387-389.

Hultin MB. Fibrinogen and factor VII as risk factors in vascular disease. In: Coller BS, ed. Progress in haemostasis and thrombosis; volume 10. London: WB Saunders Company, 1991.

Hunt BJ. The relation between abnormal hemostatic function and progression of coronary disease. Curr Opin Cardiol 1990; 5: 758-765.

Hurst JW, Schlant RC, Rackley CE, Sonnenblick EH, Wenger NK, eds. The Heart, Arteries and Veins. New York: McGraw Hill, 1990.

Jemmott JB, Locke SE. Psychosocial factors, immunologic mediation and human susceptibility to infectious diseases, how much do we know? Psychol Bul 95; 1984: 78-108.

Jern $C$, Erikson $E_{*}$ Tengborn $L$, Risberg $B$, Wadenvik $H$, Jern $S$. Changes of plasma coagulation and fibrinolysis in response to mental stress. "Thromb Haemostas $1989 ; 62: 767-71$.

Jonasson L, Holm J, Hansson GK. Cyclosporin A inhibits smooth muscle proliferation in vascular response to injury. Proc Natl Acad Sci USA 1988; 1985: 2303-2306.

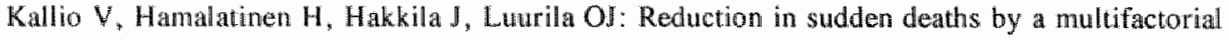
intervention program after acute myocardial infarction. Lancet 1979; ii: $1091-1094$.

Kannel WB, Wolf PA, Castelli WP, D'Agostino RB. Fibrinogen and risk of cardiowascular disease; the Framingham study. JAMA 1987; 258:1183-1186.

Karasek R, Theorell T. Healthy Work. New York: Basic Books, 1990.

Kasl S, Gore S, Cobb S. The experience of loosing a job; reported changes in health, sypmtoms and illness behavior. Psychosom Med 1975; 37: 106-122. 
Kellerman JJ, Braunwald E. Silent myocardial ischemia, a critical appraisal; vol 37. Busel: Karger, 1990.

Kirschstein W, Simianer S, Dempfle CE et al. Impaired fibrinolytic capacity and tissue plasminogen activator release in patients with restenosis after percutaneous transhuminal coronary angioplasty (PTCA). Thromb Haemostas 1989; 62: 772-775.

Klaeboe G, Otterstad JE, Wimsnes T, Espeland N. Predictive value of prodromal symptoms in myocardial infarction. Acta Med Scand 1987; 222: 27-30.

KIeinbaum DG, Kupper LL: Applied regression analysis and other multivariable methods. North Scituate: Duxbury Press, 1979.

Kluft C, Verheijen JH, Leiden Fibrinolysis Working Party. Blood collection and handling procedures for the assessment of tissue type plasminogen activator and plasminogen activator inhibitor 1.

Fibrinolysis 1990; 4 sup 2: 155-161.

Koskenvuo M, Kaprio J, Rose R, Kesaniemi A, Sarna S, Heikklia K, Langinvainio H. Hostility as a risk factor for mortality and ischaemic heart disease in men. Psychoson Med 1988; 50: 330-340.

Krantz DS, Glass DC, Snyder ML. Helplessness, stress-level and the coronary prone behavior pattern. J Exp Soc Psychol 1974; 10: 284-300.

Kuller L, Cooper M, Perper J. Epidemiology of sudden death. Arch Int Med 1972; 129: 714-719.

Kuller LH. Prodromata of sudden death and myocardial infarction. Adv Cardiol 1978; 25; 61-72.

Ladwig KH. Patient- und Artzreaktionen auf spezifische und unspezifische Warnsignale in der Vorphase eines akuten Myokardinfarktes. Verhaltensmodifikation und Verhaltensmedizin 1989; 10: $181-195$.

Ladwig KH, Kieser M, Konog J, Breithardt G, Borggrefe $M$. Affective disorders and survival after acute myocardial infarction. Results from the postinfarction late potential study. Eur Heart. I 1991; 12: 959-964.

Libby $P$, Hansson GK. Biology of disease, involvement of the immune system in human atherogertesis, current knowledge and unanswered questions. Lab Investigation 64; 1991: 5-15.

Libby P, Schartz D, Brogi D, Tanaka $H$, Clinton S. A cascade model for restenosis; a special case of atherosclerosis progression. Circulation 1992; 861 sup, III 47 III 52.

Liu MW, Roubin GS, King SB. Restenosis after coronary angioplasty. Circulation 1989; 79: 13741387.

Lloyd GG, Cawley RH. Distress or illness? A study of psychological symptoms after myocardial infarction. Br J Psychiat 1983; 142: 120-125.

Loppnow H, Libby P. Functional significance of human vascular smooth muscle cell-derived interleukin I in paracrine and autocrine regulation pathways. Exp Cel Res 1992; 198: 283-290. 
Lulofs R. Stress reactivity, Type A behaviour and vital exhausition. Ph.D Dissertation: Maastricht, 1990.

Manu $P_{i}$ Lane $T$, Mathews $D$. The frequency of chronic fatigue syndrome in patients with symptoms of persistent fatigue. Ann Int Med 109; 1988: 554-556.

Markovitz JH, Mathews KA: Platelets and coronary heart disease, potential psychophysiologic mechanisms. Psychosom Med 53:643-668, 1991.

Markowe HLJ , Marmot MG, Shipley MJ et al. Fibrinogen; a possible link between social class and coronary heart disease. BMI 1985; 291: 1312-1314.

Maslach C, Jackson SE. Maslach Burnout Inventory. Palo Alto: Consulting Psychologists Press, 1986.

Mathews $\mathrm{K}$. Coronary heart disease and Type A behavior: update on and alternative to the BoothKewley and Friedman (1987) quantitative review. Psychol Bull 1988; 104: 373-380.

Meade TW, Brozovic $M$, Chakrabarti $R$ et al. Haemostatic function and ischemic heart disease; principal results of the Northwick Park Heart Study. Lancet 1986; ii: 533-537.

Meyman FF. Over Vermoeidheid. Amsterdam: studiecentrum Arbeid en Gezondheid, 1991.

Mendes de Leon CF. Behavioral and emotional precursors of acute heart disease. Ph. D thesis, University of Texas Medical Branch. Galvestom: 1988.

Møller L, Kristensen TS. Plasma fibrinogen and ischemic heart disease risk factors. Arterioscl Thromb $1911 ; 11: 344-350$.

Montgomery G. Uncommon tiredness among under-graduates. J Couns Clin Psychol 51; 1983: 517 525.

Morrison JD. Faligue as a persisting complaint in family practice. J Family Practice 1980; 10: 795 801.

Nachman RL, Hajjar KA, Silverstein RL, Dinarello CA. Interleukin 1 induces endothelial cell synthesis of plasminogen activator inhibitor. J Exp Med 1986; 163: 1595-1600.

Nixon O. Exhaustion: cardiac rehabilitation's starting point. Physiotherapy 1986; 72: 224-228.

Norusis MJ. SPSS/PC+ ${ }^{\mathrm{m}}$, Chicago: SPSS Inc., 1986.

Munkvad S, Gram J, Jespersen J. A depression of active tissue plasminogen activator in plasma characterizes patients with unstable angina pectoris who develop myocardial infarction. Eur Heart J $1990 ; 11: 525-528$.

Olofson BO, Dahlen G, Nulsson TKK. Evidence of increased levels of plasminogen activator inhibitor and tissue plasminogen activator in plasma of patients with angiographically verified coronary artery disease. Eur Heart J 1989; 10:77-82. 
Oseroff A, Krischmamurti C, Hassett A, Tang D, Alving B. Plasminogen activator and plasminogen. activator inhibitor activities in men with coronary artery disease. J Lab Clin Med 1990; 113: 88-93.

Paffenbarger RS, Wolff PA, Notkin J, Thorne MC. Chronic disease in former college students I:

Early precursors of fatal coronary heart disease. Am J Epid 1966; 83: 314-328.

Palmblad J, Blombäck M, Egberg N, Fröberg J, Karlson C, Levi L. Experimentally induced stress in man; effects on blood coagulation and fibrinolysis. J Psychosom Res $1977 ; 21: 87-92$.

Partinen M, Putkonen P, Kaprio J, Koskenvuo M, Hilakivi I. Sleep disorders in relation to coronary heart disease. Act Med Scand 1982; 660 suppl: 69-83.

Pedhazur EJ. Multiple regression in behavioral research, 2nd edition. New York: Holt, Rinehart \& Winston, 1982.

Pickering TG. Should studies of patients undergoing coronary angiography be used to evaluate the role of behavioral risk factors for coronary heart disease.

Pietraszek MH, Takada $Y$, Nishimoto $M$, Ohara $K$, Ohara $K$, Takada A. Fibrinolytic activity in depression and neurosis. Thromb Res 1991; 63: 661-666.

Polzien P, Walter J. Das pseudoneurasthenische Syndrom in Frühstadium der Koronarsclerose. Münchener Medizinischen Wochenschrift 1971; 44: 1453-1456.

Pines A, Aronson E. Career Burnout: causes and cures. New York: The Free Press, 1988.

Ragland DR, Brand RJ. Type A behavior and mortality from coronary heart disease. New Engl J Med 1988; 318: 65-69.

Ragland DR, Helmer DC, Seeman TE. Patient selection factors in angiographic studies, a conceptual formulation and empirical test. J Beh Med 14, 541-553, 1991.

Raphael K. Recall bias: a proposal for assessment and control. Int J Epid 1987; 16:167-170.

Ray C. Chronic fatigue syndrome and depression; conceptual and methodological ambiguities. Psychol Med 1991; 21: 1-9.

Reed DM, LaCroix AZ, Karasek RA, Miller D, MacLean CA. Occupational strain and the incidence of coronary heart disease. Am J Epidemiol 1989; 129: 495-502.

Ridker PM, Vaughan D, Stampfer MJ, Manson JE, Hennekens CH. Endlogenous tissue-type plasminogen activator and risk of myocardial infarction. Lancet 1993; 341: 1165-1168.

Rissanen V, Romo M, Siltanen P. Premonitrory symptoms and stress factors preceding sudden death from ischaemic heart disease. Act Med Scand 1978; 204: 389-396.

Robins LN, Helzer JE, Ratcliff KS, Seyfried W. Validity of the Diagnostic Interview Schedule, version III; DSM-III diagnoses. Psychol Med 1982; 12: 855-870. 
Romo M: Factors related to sudden death in acute ischemic bear disease: A community study in Helsinki. Acta Medica Scand 1973: 5-92.

Rose GA, Blackburn H, Gillum RF, Prineas R. Cardiovascular survey methods, 2nd edition. Geneva: Word Health Organization, 1982.

Rosengren $A_{y}$ Tibblin $G$, Wilhelmsen $L$. Self-perceived psychological stress and incidence of coronary artery disease in middle aged men. Am J Cardiol 1991; 68: 1171-1175.

Rosenman R, Brand R, Jenkins C et al. Coronary Heart disease in the Western Collaborative Group Study: Final follow-up experience of 8/2 years. JAMA 1975; 233: 872-877.

Ross R. The pathogenesis of atherosclerosis; un update. N Engl J Med 1986; 314: 488-500.

Rothman KJ: Madern epidemiology. Baston, Toronto: Little, Brown \& Comp, 1986, 102-109.

Ruberman $W$, Weinblatt E, Goldberg J, Chaudhary B. Psychosocial influences on mortality after myocardial infarction. New Engl J Med 1984; $311: 552-559$.

Rupprecht HJ, Brennecke R, Kottmeyer M., et al. Short- and long-term outcome after PTCA in patients with stable and unstable angina. Eur Heart J 1990; 11: 964-973.

Schleifer S, Macari-Hinson M, Coyle D, Slater W, Kahn M, Gorlin R, Zucker $H$. The nature and course of depression following myocardial infarction. Arch Int Med 1989; 149: 1.785-1789.

Selye H. The stress of life. New York: McGraw Hill, 1977.

Serruys PW, Luijten HE, Beatt KJ et al. Incidence of restenosis after successful coronary angioplasty: a time related phenomenon. A quantitative angiographic study in 342 consecutive patients at $1,2,3$, and 4 months. Circulation 1988; 77: 861-871.

Serruys PW, Rensing BJ, Luijten HE, Hermans WRM, Beatt KJ: Restenosis following coronary angioplasty. In Meier B (ed), Interventional Cardiology. Bern: Hogrefe \& Huber, 1990, 79-115.

Sthekelle RB, Ostfeld AM. Psychometric evaluations in cardiovascular epidemiology. Ann NY Acad Sci 1965; 126: 696-705.

Shephard R, Kavanagh T, Klavora P. Mood states during postcoronary cardiac rehabillitation. I Cardiopuln Rehab 1985; 5: 480-484.

Shirom A, Burnout in work organizations. In: CL Cooper, I Robertson, eds: International review of Industrial and Organizational Psychology, New York: J Wiley, 1989.

Siegrist J. Sleep disturbances and cardiovascular risk. In: J Peter, T Podszus, P von Weichert eds. Sleep related disorders and internal diseases, Berlin: Springer, 1987.

Siegrist J, Peter R, Junge A, Cremer P, Seidel D. Low status control, high effort at work and ischaemic heart disease: prospective evidence from blue collar men. Soc Sci Med 1990; $31: 1127$ 1134. 
Siegrist J. Contributions of sociology to the prediction of heart disease and their inplications for public health. Eur J Publ Health 1991; 1: 10-21.

Sihm I, Dehlholm G, Hansen E, Gerdes L, Faergeman O. The psychological work environment of younger men surviving acute myocardial infarction. Eur Heart J 1991; 12; 203-209.

Silverstone P. Depression and outcome in acute myocardial infarction. Br Med J 198\%; 294: 219-220.

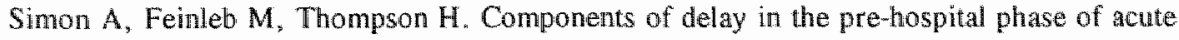
myocardial infarction. Am J Cardioll 1972; 30:476-481.

Stone MC, Thorp JM, Plasma fibrinogen; a major coronary risk factor. J R Coll Gen Pract 1985; 35; $565-569$.

Stern $D$, Nawroth P, Handley D, Kisiel W. An endothelial cell-dependent pathway of coagulation. Proc Natl Acad Sci USA 1985; 82: 2523-2527.

Sternberg EM, Glowa J, Smith M et al. Corticotrophin releasing hormone related behavioral and neuroendocrine responses to stress in Lew is and Fischer rats. Brain Res 570; 1992: 54-60.

Stowers M, Short D. Warning symptoms before nyocardial infarction. Br Heart J 1970; 32 : 833838.

Straus SE, Dale JK, Tobi M, Lawley T, Preble O, Blease M, Hallahan C, Henle W. Acyclovir treatment of the chronic fatigue syndrome. N Engl J Med 1988; 319: 1692-1698.

Thiele $\mathbb{R}$, Simon $H$, Thiele G. Stressfactoren bei Herzinfarktpatienten vor Eintritt des Herzinfarktes. Zeitschr Gesamte Inn Med 1985; 40: 483-488.

Tosato G, Straus S, Henle W, Pike SE, Blease RM. Characteristic T Teell dysfunction in patients with chronic active Epstein-Barr vïrus infection (infectious mononucleosis). J Immunol 1985: 134: 3082 3088.

Trijsburg $\mathbf{R}$, Erdman $\mathbf{R}$, Duivenvoorde $\mathbf{H}$, Thiel $\$$, Verhage $F$. Denial and overcompensation in malle patients with myocardial infarction. Psychoth Psychosona 1987; 47: 22-28.

Verhagen F, Nass C, Appels A, van Bastelaer A, Winnubst I. Cross validation of the A/B typology in the Netherlands. Psychother Psychosom 1980; 34: 178-186.

Vossen $\mathbb{R}$. Fatty acid modification and endothelial cell reactivity. Dissertation: Maastricht, 1993.

de Vries CJM. Structure and function of tissue-type plasminogen activator; a molecular biological approach. Ph.D. Dissertation: University of Amsterdam, 1990.

Webster MWI, Chesebro JH, Smith HC et al. Myocardial infarction and coronary artery acclusion; a 5-year angiographic study (abs). I Am Coll Cardiol 1990; 15 sup.

Wessely $S$, Powell $R$. Fatigue syndrome; a comparison of 'postviral' fatigue with neuromuscular and affective disorder. J Neurol Neurosurg Psychiat 1989; $52: 940-948$. 
Wilhelmsen $L$, Syardsudd $K$, Korsan-Bengtsen $K$ et al. Fibrinogen as a risk factor for stroke and: myocardial infarction. N Engl J Med 1984; 311 : 501 $\times 505$.

Williams R. Refining the Type A hypothesis: emergence of the hostility complex. Am J Cardiol 1987,60 suppl $\mathbb{J}: 27-32$.

Williams RB, Barefoot JC, Haney TL et al. Type A behavior and angiographically documented coronary atherosclerosis in a sample of 2,289 patients. Psychosom Med 50;1988: 139-152.

Williams RB, Suarez EC, Kuhn CM, Zimmerman EA, Schanberg SM. Blobehavioral basis of coronary prone behavior in middle aged men; part 1, evidence for chronic SNS activation in Type As. Psychosom Med 1991; 53: 517-527.

Wolf S. PSychosocial forces in myocardial infarction and sudden death. Circulation 1969; 40: 74-83.

Yeung A, Vekshtein V, Krantz D, Vita J, Ryan Th, Ganz P, Selwyn A. The effect of atherosclerosis on the vasomotor response of coronary arteries to mental stress. New Engl J Med 1991; 325: 1551 1556.

Zakowski S, McAllister G, Deal M, Baum A. Stress, reactivity, and immune function in healthy men. Health Psychol $1992 ; \| 1: 223-232$.

Zeiher AM, Drexler H, Wollschlager H, Just H. Modulation of coronary wasomotor tone in human progressive endothelial dysfunction with different early stages of coronary atherosclerosis. Circulation $1991 ; 83: 391-401$. 


\section{Summary.}

The present thesis addresses the relationship between vital exhaustion and coronary artery disease. The occurrence of new cardiac events after successful coronary balloon dilatation (angioplasty) is chosen as a model to investigate this issue prospectively.

Excessive fatigue is one of the most prevalent premonitory symptoms of myocardial infarction and sudden cardiac death. In the first chapter, an overview is provided of cardiological, psychiatric, and psychological explanations of fatigue prior to clinically manifestations of cardiac disease. Arguments are given why the label "vital exhaustion" is chosen for this characteristic state of fatigue. Vital exhaustion consists of three typical components: extreme tiredness, increased irritability, and demoralization. Previous studies have shown that vital exhaustion is an independent risk indicator of myocardial infarction. However, these studies could not rule out whether the association between vital exhaustion and future myocardial infarction is explained by confounding due to (subclinical) coronary artery disease. That is, subjects whose coronary arteries were affected by atherosclerotic narrowing, and who were for that reason at the highest risk of developing myocardial infarction, may also have reported feelings of exhaustion more often. The plausibility of this objection will be investigated in chapters 3 and 4.

An outline of the theory of the development of vital exhaustion is presented in chapter 1. It is proposed that vital exhaustion is the end stage of prolonged psychological tension, in which the individual can no longer handle the demands of daily life stressors. Certain personality types who experience increased levels of psychological stress (e.g., Type As, or hostile persons) are more prone to become exhausted than individuals who encounter less stressors. Support for this notion was found in two previous case-control studies which demonstrated that vitally exhausted Type As were at the highest risk of myocardial infarction. The contribution of stressful life-events, overwork, and lack of sleep, in the development of vital exhaustion and future cardiac disease, as well as the differences and similarities between vital exhaustion, depression, burnout, and the chronic fatigue syndrome are discussed. Chapter 1 concludes with the research questions and related hypotheses of the current thesis.

The methods and rational for the studies reported in chapters three through six are outlined in chapter 2. Two lines of investigation were carried out. In the first approach, vital exhaustion was assessed in consecutive patients referred to the department of Cardiology in Maastricht for coronary angiography. This allowed an 
investigation of the relationship between vital exhaustion and the severity of coronary artery disease (chapter 3). Coronary disease was treated with Percutaneous Transluminal Coronary Angioplasty (PTCA) in a subset of these coronary angiography patients. Patients who had PTCA were followed up for one year and a half, while changes in exhaustion (chapter 4) and the occurrence of new cardiac events (chapter 5) were monitored.

In the second approach we made use of healthy subjects in order to examine if blood-clotting factors are elevated in vitally exhausted individuals in comparison with not exhausted individuals. This study is reported in chapter 6 .

As is discussed in chapter one, several studies have shown that exhaustion is of predictive value for first myocardial infarction. However, these studies could not exclude that the state of exhaustion prior to myocardial infarction was the result of underlying coronary artery disease. This issue is addressed in chapter three. If exhaustion and the extent of coronary disease are found to be strongly related, then there would be little need to search for psychological explanations for the extreme tiredness before myocardial infarction. To investigate this, the extent of coronary artery disease and cardiac pump function were related to feelings of exhaustion in 307 patients who underwent coronary angiography. Exhaustion was assessed by means of the Maastricht Questionnaire.

It was found that exhaustion was not linearly related to the extent of coronary artery disease $(F=1.17 ; p=0.15$ ). Furthermore, a poor left ventricular function did not relate to the exhaustion scores. On the other hand, clinical variables (duration of complaints, exercise performance, peripheral vascular disease, and dyspnea), the use of medication (nitrates, B-blocking agents, calcium antagonists, and diuretics), and demographic characteristics (gender and education) were associated with exhaustion scores. Multiple regression analysis showed that gender and education were the best predictors of exhaustion scores $\left(\mathrm{R}^{2}=0.09 ; \mathrm{F}=8.1 ; \mathrm{p}<0.001\right)$.

It is concluded that neither the extent of coronary artery disease nor a reduced pump function of the heart is related to feelings of exhaustion in patients referred for coronary angiography. Therefore, the previously reported association between exhaustion and future myocardial infarction is not likely to be caused by underlying coronary disease.

In order to assess the relationship between vital exhaustion and underlying coronary artery disease in a more controlled (quasi-experimental) way, we examined changes in vital exhaustion following restoration of coronary blood-flow in chapter four. If vital exhaustion markedly decreases following improvement of coronary blood-flow, there would be no need for further studies that investigate a psychological origin of exhaustion in patients at risk of myocardial infarction.

For this purpose, patients who had a successful elective PTCA were evaluated on feelings of exhaustion on admission, two weeks after discharge, and six months after discharge.

Successful PTCA resulted in a significant decrease of exhaustion scores ( $p<$ $0.001)$. However, the majority of patients who were exhausted before PTCA remained 
exhausted after PTCA. That is, exhaustion was present in $75 \%$ of the patients before PTCA and in $65 \%$ two weeks after PTCA, which indicates that restoration of coronary perfusion by successful PTCA did not substantially reduce the number of exhausted patients. At six months, exhaustion was present in $60 \%$ of the patients, and there was no difference between patients with and without typical anginal complaints at that time.

In conclusion, successful PTCA does not substantially ameliorate feelings of exhaustion. This is additional support for the notion that impaired coronary perfusion can not account for the extreme fatigue prior to myocardial infarction.

In chapter five we investigated the predictive value of vital exhaustion for the occurrence of new cardiac events after PTCA. Furthermore, it was examined whether this association continued to be of importance if the severity of underlying coronary artery disease was explicitly controlled for.

Patients with a successful PTCA were followed up during 1.5 years. A new cardiac event was defined as present if one of the following end-points occurred: cardiac death, myocardial infarction, coronary bypass surgery, repeat-PTCA, increase in coronary atherosclerosis, or new anginal complaints with documented ischemia. Vital exhaustion was assessed using the Maastricht Questionnaire two weeks after hospital discharge.

Fifteen $(35 \%)$ of the 43 exhausted patients experienced a new cardiac event, while $14(17 \%)$ of the 84 not exhausted had a new cardiac event $(\mathrm{OR}=2.7 ; \mathrm{CI}=1.1$ $6.3 ; p=0.02)$. Multiple logistic regression analysis revealed that vital exhaustion was of predictive value when other significant risk factors for new cardiac events were controlled for (i.e., severity of coronary artery disease, and hypercholesterolemia).

It is concluded that vital exhaustion is associated with an unfavorable clinical course after successful PTCA. The predictive value of vital exhaustion is independent of the severity of coronary atherosclerosis and other known risk factors for progression of coronary artery disease.

Chapter 6 reports about a possible mechanism that might explain the relationship between wital exhaustion and future cardiac events. Both prolonged psychological stress as well as blood-coagulation and fibrinolysis have been found to elevate the risk of future myocardial infarction. We therefore investigated a well-defined group of healthy subjects who were exhausted as a consequence of prolonged psychological stress $(\mathrm{N}=15)$ and compared them with a group of definitely non-stressed subjects $(\mathrm{N}=15)$ measuring coagulation factors (VIIc, VIIIc, and fibrinogen) and fibrinolytic parameters (tissue plasminogen activator (t-PA) antigen and plasminogen activator inhibitor (PAI) activity). Subjects were matched on age, and blood samples were collected twice to avoid confounding by intra-individual fluctuations.

PAI activity was significantly higher in the exhausted group than in the not exhausted group (medians 13.0 and $6.0 \mathrm{au} / \mathrm{ml}$, respectively). No differences were observed in factor VIIc, factor VIIIc, fibrinogen, and t-PA antigen. The groups did not differ in smoking status, blood pressure, cholesterol, triglycerides, hematocrit, platelet count, leucocyte count, neutrophil count, or hepatic and renal function. 
These data suggest that the fibrinolytic system is dysfunctional in vitally exhausted individuals. The relationship between psychological stress and future myocardial infarction may in part be mediated in part by an imbalance between coagulation and fibrinolysis.

A psychobiological model that might explain the relationship between vital exhaustion and future coronary artery disease is discussed in chapter 7 . It is argued that personality characteristics such as hostility and Type A behavior promote the gradual progression of coronary disease. In the setting of moderately developed coronary lesions, a state of vital exhaustion will add substantially to the occurrence of acute coronary syndromes. The model incorporates lesional growth, age, hostility, and vital exhaustion, and postulates that especially the exhausted Type A person at ages $<55$ years is at elevated risk of developing an acute coronary syndrome. In addition to the importance of blood-clotting and fibrinolytic factors, it is suggested that immunologically regulated cell growth mediators might play a role in the relationship between vital exhaustion and future cardiac events. Research on the effects of psychological factors on immunological aspects of atherosclerosis might indicate a new area of research in cardiovascular behavioral medicine. 


\section{Samenvatting.}

Dit proefschrift behandelt de relatie tussen vitale uitputting en coronaire hartziekte. Het optreden van nieuwe cardiale gebeurtenissen na een succesvolle Dotterbehandeling is gekozen als model om deze samenhang longitudinaal te onderzoeken.

Extreme vermoeidheid is een van de meest voorkomende symptomen die voorafgaan aan het hartinfarct en de plotselinge dood. Hoofdstuk $l$ geeft een overzicht van de cardiologische, psychiatrische en psychologische verklaringen voor de extreme vermoeidheid die veelal vooraf gaat aan klinisch manifest hartlijden. Aangevoerd wordt waarom er voor 'vitale uitputting' gekozen is als naamgeving van deze karakteristieke vermoeidheidstoestand. Vitale uitputting bestaat uit drie componenten: extreme vermoeidheid, toegenomen prikkelbaarheid en demoralisatie. Eerdere onderzoekingen hebben aangetoond dat vitale uitputting een onafhankelijke risicoindicator is van het hartinfarct. Deze studies konden echter niet uitsluiten dat de samenhang tussen vitale uitputting en het later optreden van een hartinfarct toe te schrijven was aan subklinische atherosclerose van de kransslagaders. Met andere woorden, het zou kunnen zijn dat personen wier kransslagaders door atherosclerose waren aangetast en die derhalve een verhoogd risico hadden op een hartinfarct, naar verhouding meer gevoelens van uitputting rapporteerden. De aannemelijkheid van dit bezwaar wordt onderzocht in hoofdstukken 3 en 4 .

Het ontstaan van vitale uitputting wordt besproken in hoofdstuk 1 , Verondersteld wordt, dat vitale uitputting het eindstadium is van voortdurende psychische belasting waarbij de persoon in kwestie de energie niet meer heeft om de dagelijkse spanningen het hoofd te bieden. Er zijn bepaalde persoonlijkheidstypes die relatief veel psychische stress meemaken (Type A of vijandige personen) en dientengevolge meer kans hebben om uitgeput raken dan zij die minder vaak gespannen situaties tegenkomen. Er is ondersteuning voor deze hypothese gevonden in twee voorgaande 'case-controle' studies, waarin werd gevonden dat vitaal uitgeputte Type A personen het meeste kans hadden op een hartinfarct. Eveneens wordt in hoofdstuk 1 besproken wat de invloed is van stresserende levensgebeurtenissen, overwerk en een gebrek aan slaap op het ontstaan van vitale uitputting, alsook de verschillen en overeenkomsten tussen vitale uitputting, depressie, 'opgebrand zijn' en het chronisch vermoeidheidssyndroom. Hoofdstuk 1 sluit af met de vraagstellingen van onderhavig proefschrift en de bijbehorende te toetsen hypothesen. 
De methode en rationale voor de in de hoofdstukken 3 tot en met 6 te bespreken studies, worden uiteengezet in hoofdstuk 2. In principe is gebruik gemaakt van twee lijnen van onderzoek. Bij de eerste benadering is vitale uitputting bepaald onder opeenvolgende patiënten die een coronair-angiogram ondergingen in het Academisch Ziekenhuis te Maastricht. Dit maakte een studie mogelijk naar de relatie tussen de ernst van vitale uitputting en de mate van atherosclerose in de kransslagaders (hoofdstuk 3). In een deel van deze hartpatiënten werd het kransslagaderlijden behandeld met Percutane Transluminale Coronaire Angioplastiek (PTCA, ook wel 'Dotter-behandeling', naar de uitvinder van deze ballondilatatie techniek). Deze PTCA patiënten zijn gedurende anderhalf jaar gevolgd waarbij zowel de veranderingen in uitputting (hoofdstuk 4), als het ontstaan van mieuwe cardiale gebeurtenissen (hoofdstuk 5) werden geregistreerd.

Bij de tweede benadering is gebruik gemaakt van gezonde proefpersonen met het doel te onderzoeken of bloedstollingsfactoren verhoogd zijn bij vitaal uitgeputte individuen. De rapportage van de resultaten is te vinden in hoofdstuk 6.

In hoofdstuk 1 zijn diverse studies beschreven die de voorspellende waarde van vitale uitputting voor het ontstaan van een eerste hartinfarct aantonen. Deze studies konden echter niet uitsluiten dat de toestand van uitputting voor het hartinfarct het gevolg was van onderliggende ziekte van de kransslagaders, hetgeen onderwerp van studie is in hoofdstuk 3. Als er een sterke samenhang is tussen de ernst van uitputting en de mate van atherosclerose, dan is er weinig reden om naar een psychologische verklaring te zoeken voor de extreme vermoeidheid die aan het hartinfarct vooraf gaat. Om dit nader te onderzoeken is bij 307 patiënten die een coronair-angiogram ondergingen de ernst van vitale uitputting, als gemeten met de Maastrichtse Vragenlijst, gerelateerd aan de mate van het kransslagaderlijden en de kwaliteit van de pompfunctie van het hart.

Er bleek geen lineaire relatie te bestaan tussen vitale uitputting en het aantal vernauwde coronairvaten $(F=1.17 ; p=0.15)$. Bovendien was er geen samenhang tussen een verminderde pompfunctie van het hart en de ernst van vitale uitputting. Vitale uitputting was daarentegen wel geassocieerd met klinische kenmerken van de patiënten (duur van hartklachten, fysieke inspanbaarheid, perifeer vaatlijden en kortademigheid), het gebruik van diverse medicamenten (nitraten, B-blockers, calcium antagonisten en diuretica), en demografische karakteristieken (geslacht en opleiding). Met behulp van multiple regressie analyse werd aangetoond dat geslacht en opleiding de beste voorspellers van de uitputtingscores waren $\left(R^{2}=0.09 ; p<0.001\right)$.

Uit deze resultaten kan worden afgeleid bat bij patiënten die verwezen zijn voor coronaire angiografie, vitale uitputting niet beduidenswaardig samenhangt met dat de mate van atherosclerose van de kransslagaders, noch met een verminderde pompfunctie van het hart. Derhalve is het niet waarschijnlijk, dat onderliggende atherosclerose de oorzaak is van de voornoemde observatie dat vitale uitputting van voorspellende waarde is voor een toekomstig hartinfarct.

Hoofdstuk 4 beschrijft een studie die werd uitgevoerd met als doel de relatie tussen vitale uitputting en onderliggend kransslagaderlijden op een meer gecontroleerde 
(quasi experimentele) wijze te onderzoeken. Nagegaan werd of de vitale uitputting substantieel verandert als gevolg van een herstel van de doorbloeding van de kransslagaders. Indien vitale uitputting aanzienlijk zou afnemen als gevolg van herstelde coronaire perfusie, dan zou er weinig aanleiding zijn voor verdere studies naar een eventueel psychologische oorsprong van de uitputting van patiënten die risico lopen op een hartinfarct.

Met dit oogmerk werd vitale uitputting bepaald in patiënten bij wie een succesvolle Dotter-behandeling werd uitgevoerd op drie tijdstippen: voor de behandeling, twee weken na ontslag uit het ziekenhuis, en een half jaar na de behandeling, waarbij spoedbehandelingen buiten beschouwing werden gelaten.

Succesvolle Dotter-behandeling resulteerde in een significante vermindering van uitputtingscores $(p<0.001)$. Het merendeel van de patiënten bleef echter uitgeput na de succesvolle Dotter-behandeling, waarbij het percentage uitgeputten van $75 \%$ voor behandeling, afnam tot $65 \%$ twee weken na het Dotteren. Dit geeft aan dat na herstel van de doorbloeding van de kransslagaders het aantal uitgeputte patiënten nauwelijks teruggebracht werd. Vitale uitputting was in $60 \%$ van de gevallen na zes maanden nog aanwezig, waarbij er geen verschil in uitputting gevonden werd tussen patiënten met of zonder pijn op de borst die typerend is voor kransslagaderlijden.

De conclusie van het voorgaande is dat het aantal vitaal uitgepulte patiènten niet noemenswaardig afneemt na een succesvolle Dotter-behandeling. Dit ondersteunt de veronderstelling dat verminderde doorbloeding van de kransslagaders geen adequate verklaring biedt voor de prevalente aanwezigheid van uitputting voorafgaand aan het hartinfarct.

In hoofdstuk 5 werd de voorspellende waarde van vitale uitputting onderzocht op het ontstaan van nieuwe cardiale gebeurtenissen na een Dotter-behandeling. Bovendien werd nagegaan of deze samenhang van belang bleef bij expliciete controle voor de invloed van onderliggend kransslagaderlijden.

Patiënten die een succesvolle Dotter-behandeling ondergingen werden gedurende anderhalf jaar gevolgd, waarbij van een nieuwe cardiale gebeurtenis gesproken werd indien een van de volgende omstandigheden zich voordeed: cardiale dood, hartinfarct, coronaire bypass operatie, herhaalde. Dotter-behandeling, toename van coronaire atherosclerose, of nieuwe klachten yan pijn op de borst met gedocumenteerde nieuwe myocardiale ischemie. De aanwezigheid van vitale uitputting werd twee weken na ontslag uit het ziekenhuis bepaald met behulp van de Maastrichtse Vragenlijst.

Vijftien van de 43 uitgeputte patiënten (35\%) overkwam een nieuwe cardiale gebeurtenis, terwijl dit bij $14(17 \%)$ van de 84 niet uitgeputten het geval was (OR = $2.7 ; \mathrm{CI}=1.1-6.3 ; \mathrm{p}=0.02$ ). Multiple logistische regressie analyse bevestigde de voorspellende waarde van vitale uitputting indien er gecontroleerd werd voor de andere betekenisvolle predictoren van nieuwe cardiale gebeurtenissen (i.c., de mate van kransslagaderlijden en hypercholesterolemie).

Deze studie toont aan, dat vitale uitputting geassocieerd is met een ongunstig klinisch beloop na een aanvankelijk succesvolle Dotter-behandeling. De voorspellende waarde is niet toe te schrijven aan de mate van coronaire atherosclerose of andere bekende predictoren van de progressie van atherosclerose. 
Hoofdstuk 6 gaat in op een mogelijk mechanisme dat de relatie tussen vitale uitputting en toekomstige cardiale incidenten kan verklaren. Het is bekend dat zowel bloedcoagulatie en fibrinolyse, als voortdurende psychologische spanning de kans op een hartinfarct vergroten. Derhalve werden coagulatie factoren (VIIc, VIIIc, en fibrinogeen) en fibrinolytische parameters (Plasminogeen Activator Inhibitor activiteit (PAI) en t-PA antigeen) bepaald in een welomschreven groep gezonde proefpersonen die uitgeput waren als gevolg van voortdurende psychische belasting $(\mathrm{N}=15)$. Deze werd vergeleken met een op overeenkomende leeftijd geselecteerde controlegroep $(\mathrm{N}=15)$. De bloedbepalingen werden twee maal uitgevoerd ter voorkoming van artefacten veroorzaakt door intra-individuele fluctuaties van de betreffende stollingsparameters.

PAI activiteit was significant verhoogd in de uitgeputte groep in vergelijking tot de niet uitgeputte groep (mediaan $=13.0$ and $6.0 \mathrm{au} / \mathrm{ml}$, respectievelijk). Er werden geen betekenisvolle verschillen gevonden in factor VIIc, factor VIIIc, fibrinogeen en tPA antigeen. De groepen onderscheidden zich niet in rookgedrag, bloeddruk, noch in de spiegels van cholesterol, triglyceriden, of andere standaard hematologische bepalingen.

Deze gegevens geven aanwijzingen voor een disfunctioneel fibrinolytisch systeem in uitgeputte individuen. De relatie tussen voortdurende psychologische stress en het ontstaan van het hartinfarct zou derhalve deels gemedieerd kunnen worden door een verstoorde balans tussen bloed coagulatie en fibrinolyse.

In hoofdstuk 7 wordt een psychobiologisch model beschreven dat een mogelijke verklaring zou kunnen bieden voor de samenhang tussen vitale uitputting en toekomstige aandoeningen van de kransslagaders. Verondersteld wordt, dat persoonlijkheidskenmerken zoals het Type A gedragspatroon en vijandigheid de geleidelijke progressie van coronaire atherosclerose bevorderen. Bij aanwezigheid van een beperkt ontwikkelde beschadiging van de kransslagader, verhoogt een toestand van vitale uitputting de kans op een acute hart aandoening aanzienlijk, als gevolg van een toegenomen risico op afsluiting van een kransslagader. De relatie tussen atherosclerose, leeftijd, vijandigheid, en vitale uitputting worden in één model geïntegreerd, waarin aannemelijk wordt gemaakt dat een verhoogd risico op een hartinfarct met name aanwezig is bij uitgeputte Type A personen die jonger zijn dan 55 jaar. Naast het belang van bloedstollings en fibrinolytische factoren, worden suggesties gedaan aangaande de rol van immunologisch gereguleerde groei-mediatoren in de samenhang tussen vitale uitputting en het ontstaan van toekomstige cardiale gebeurtenissen. Onderzoek naar de effecten van psychologische factoren op immunologische aspecten van atherosclerose zou een mogelijk nieuw onderzoeksgebied zijn voor de cardiovasculaire behavioral medicine. 


\section{Appendix I.}

Case reports of the prodromal psychological state before myocardial infarction.

case $\mathbb{1}$.

Mr. P. is 45 years old. At the age of 21 he started a garage, where he worked 14 hours daily. After three years he was forced to close it down because of increasing complaints of low back pain. He was unemployed for six months. Thereafter, he started a cafeteria and worked about 70 hours a week. Two years later the neighborhood was reconstructed and he lost a large number of his customers. He asked the bank for a loan but the bank refused. So, he had to close the cafeteria. Becoming more and more disabled by his low back pain he sought for an administrative job for two years. He got a position as a custodian in a high school. This school was located in three provisional buildings at different locations in the town. After some years the school received permission to move into a new building which meant that one of the three custodians had to leave the job.

Mr. P. did not have any say over the decision as to who had to be dismissed. The last two months he became more and more tired, slept badly, and often woke up during the night. He had the habit of going to the sitting-room to smoke some cigarettes. He was developing a feeling of hopelessness, became easily irritated and did not feel well. Sometimes he experienced unusual chest pain. When he watched the $8 \mathrm{pm}$ television news, he repeatedly fell into a sleep that is very deep, making it even impossible for his wife to wake him up. This was embarrassing to him and he decided to use an uncomfortable kitchen-chair to watch television.

Lately, his dog ate something poisoned in a nearby field. He accused some hunters in a local newspaper of poisoning dogs and asked the police to help him fight this injustice. The police responded that there was absolutely no evidence for his claim and that he would be better off if he did avoid a libel suit. This response made him feel completely helpless. Five days later he suffered a heart attack.

case 2.

Mr. V. is a 36 years old former boxing champion, who worked as a cook in a tourist hotel. During the season he was on the job for $15-16$ hours a day. He had been overworked before six years. He once got so angry at a waiter who did not take the dishes out of the kitchen in time that he put him on the cooking-range for a full minute. $\mathrm{MrV}$. was fired for that reason.

After one year he was again making long hours in another restaurant. At the end of the third season he became listless and tired. He avoided company and no longer joined his colleagues during the coffee breaks. He wanted peace of mind. He became 
silent and observed that he was often thinking about his father who died a year and a half earlier. He started to read the obituaries in the local newspaper, something that he had never done before. He used to have many animals at home, which he started to sell one after the other. When he had sold all his birds, dogs, rabbits, and guinea pigs, he told his wife that he wanted to divorce her. His wife arranged a meeting with a psychiatrist. When asked why he wanted a divorce, Mr. V. could not give an answer; he just wanted it. This was one week before he suffered a myocardial infarction. 


\section{Appendix II.}

\section{The Maastricht Questionnaire (Dutch/English).}

Wilt U hierondier angeven hoe U xich de afgelopen drie maanden heeft gevoeld? Er nijn geen goedo of verkeerde antwoorden. U kunt de vragen met "ia", "?", of "nee" besintwoorden.

Voorbeeld: Voelt U zich wak moe?

Als U die vriag met IIA wilt beantwoorden, omcirkel. dian JA in de eerste kolom.

Dus zo.

Als U de vraag met NEE wilt beantwoarden,

omcirkel dar NEE in de laatste kolorr.

Dus 70

Weet $U$ het niet, of kunt $U$ het riet met

JA? NEE

Uzelf eens worden, omoirkel dan het vragteken.

Dus zo.

1. Voelt $\mathrm{U}$ zich wakk moe?

IA $\because \mathrm{NEH}$ Do you often fieet wired?

2. Hebt $\mathrm{U}$ er vark moente mee om in te slapen?

JA ? NEE Do you aften have trouble falling asleep?

3. Wordt $U$ 's machts vaak wakker?

IA? NEE

Do yot wake up repeatedly during the migh?

4. Voelt U zich wask slap

JA $?$ NEE Do you feel weak all over?

5. Hebt U het gevoel dat U de latste tijd weinig presteert?

JA ? NEE Do you have the feeling that you haven't been accompltshing much lately?

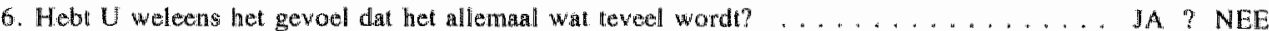

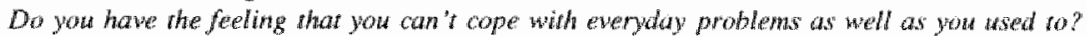

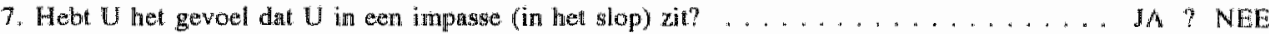
Do you believe thar you have come to a "dead ead"?

8. Voelt $\mathrm{U}$ zich de latste tijd lustelozer dan vroeger?

$\mathrm{AA} ? \mathrm{NEE}$ Do you lately feel more listless than before?

9. Ik theb net zoveel plezier in het sexuele alis vroeger? .................. JA ? NEE lenjoy sex as much as ever

10. Hebt U de laatste tijd weleens een gevoel wan hopeloosheid gehad? Have you experienced a feeling of hopelessness recently?

11. Doet $\mathrm{U}$ er nu langer over om een moeilijk probleem te begripen dan aen jaar geleden? . . JA ? NEE Does it take more to grasp a difficult problem than it did a year ago? 
12. Is het zo dal allerlè kleine dingen $U$ meer ergeren dan vroeger? Do turtle things irricate you more lately than they used to?

13. Hebt $U$ de laatste tijd weleens het werlangen voelen opkomen om het bijltie erbij neer te leggen? Do you feel you want to give up trying?

14. Ilk voel the prima JA ? NEE Ifeel fine

15. Hebt U weleens hef gevoel dat uw lichaam een batterij is, waarvan de krackt of het vermogen aan het opraken is? Do you sometimes feel that your body is like a battery that is losing its power?

16. Zou U soms weleens dood willen zijn? Would you want to be dead at times?

17. Hebt U de laatste tijd het gevoel dat U niet meer zoveel waard bent als vroeger? . . . . JA ? NEE Do you have the feeling these days that you just don"t have what it takes any more?

18. Woelt $U$ zich moedeloos? Do you feel dejected?

19. Hebt U weleens huilbuien? Do you feel like crying sometimes?

20. Wordt $\mathrm{U}$ weleens wakker met een gevoel van uitputting en vermoeidheid? Do you ever wake up with a feeling of exhaustion and farigue?

21. Is het moeilijk voor U geworden om U lang op één ding te concentreren? Do you have increasing difficulty in concentrating on a single subject for long?

22. Kunt $U$ de laatste tijd door kleine dingen soms erg geprikkeld raken? Do minor hassles irritate you more easilly the last few months?

23. Ik kan de laatste tijd soms erg opvliegend zijn JA ? NEE Do you blow up more easily than before? 


\section{Dankwoord.}

Hoewel er één auteur op de omsiag van dit proefschrift vermeld staat is het niet eén persoon geweest die het beschreven werk verricht heeft. Ik zou dan ook graag mijn erkentelijkheid betuigen aan allen die een bijdrage hebben geleverd aan de uiteindelijke totstandkoming van deze dissertatie.

Dit geldt in de eerste plaats de honderden mensen uit Maastricht en omgeving die hun medewerking aan dit onderzoek hebben verleend. Vaak was dat ten tijde van een moeilijke en onzekere levensfase. Zonder dit altruisme had dit boek er nooit geweest. Ik weet dat het velen naderhand goed gegaan is, maar sommigen gaan nog steeds gebukt onder lichamelijke of psychische moeilijkheden. Daar heeft deelname aan dit onderzoek niet veel aan kunnen doen.

Mijn promotor en begeleider Professor Ad Appels heeft mij geleerd dat het schrijven van een wetenschappelijk artikel en alles wat daaraan vooraf gaat meer dan gecompliceerd is; in ieder geval lijkt het verstandig bij voortduring alert te zijn op 'biasses' en 'confounders' van welke origine dan ook. Ik heb bijzonder goede herinneringen aan onze wandelingen langs de Jeker en later rond het Academisch Ziekenhuis in opbouw. Op gezette tijden gaf je me het nodige duwtje in de rug; het was een waardevolle tijd Ad.

Als co-promotor heeft Dr. Frits Bär mij behoed voor de valkuilen van de Cardiologie waar een welwillend Psycholoog maar al te gauw dreigt in te vallen. Commentaar van leerzaam niveau heeft dit proefschrift in ieder geval in kwaliteit doen toenemen. Beste Frits, ik heb respect voor je efficiënte en nauwgezette manier van werken. Cardiologen van jouw kaliber zijn onontbeerlijk voor de cardiovasculaire medische psychologie.

Dr. Carlos Mendes de Leon is degene geweest die dit onderzoek op de rails heeft gezet en die later vanuit Yale University actief bij de studie betrokken is gebleven. Jouw heldere denkwijze is een voorbeeld voor me Carlos en ik hoop er op zijn minst iets van overgenomen te hebben.

Met bijzonder veel plezier heb ik samengewerkt met de afdeling Hematologie van de vakgroep Interne Geneeskunde. Dr. Karly Hamulyák gaf mij alle ruimte on het onderzoek naar de bloedstollingsfactoren bij uitgeputten uit te voeren. Beste Karly, ik ben nog niet eerder iemand tegen gekomen waarmee ik met een vergelijkbaar beperkt aantal woorden tot deze productiviteit heb kunnen komen. Dit is overigens mede te danken aan de geweldige kwaliteiten van Carina Pernot die de bloedbepalingen voor haar rekening nam.

Vanzelfsprekend gaat mijn erkentelijkheid eveneens uit naar de leden van de beoordelingscommissie, bestaande uit: Prof. Dr. J. Jolles (voorzitter), Dr. A. Gorgels, Prof. Dr. M.A. van den Hout, Prof. Dr. R.S. Reneman, en Prof. Dr. J. Siegrist (Heinrich Heine Universität, Düsseldorf).

Op deze plaats wil ik, hoewel het met dit proefschrift zelf niet veel van doen heeft, mijn waardering uitspreken voor Professor Floor Kraaimaat. In de tijd dat Utrecht nog zijn standplaats was, heeft hij voor veel studenten in de Psychologie de mogelijkheid geschapen om onderzoek te doen in het Academisch Ziekenhuis aldaar. Ik ben een van de velen die daar de vruchten van heeft mogen plukken bij de afdelingen Foneatrie, 
Gynaecologie \& Obstetrie en Klinische Psychiatrie. Waarde Floor, dit is voor mij van onschatbaar belang geweest, ik ben je daarvoor meer dan dank verschuldigd.

Voorts dank ik Professor Hein Wellens en de vakgroep Cardiologie van het Academisch Ziekenhuis Maastricht voor de gastvrijheid die ik genoten heb en de leerzame ochtendbesprekingen, welke in de loop van vier jaar in mijn beleving van een aaneensluiting van onbegrijpelijk proza veranderden in leerzame bijeenkomsten. Dr. Hans de Swart dank ik voor zijn bijdrage aan twee hoofdstukken van dit proefschrift, Dr. Rob van Diest (KJinische Psychiatrie) voor het kritisch doorlezen van het gehele manuscript en Dr. Renée Vossen (Biochemie) voor haar heldere commentaar op het laatste hoofdstuk. De medewerking van de poliklinieken Cardiologie in de regio's Zuid en Midden Limburg was een voorwaarde voor de completering van de PTCA studie. Verder is de administratieve ondersteuning van Marjan Stegeman, en met name Marieke Cruip bijzonder instrumenteel geweest.

Voor de behulpzaamheid bij het recruteren van voldoende uitgeputte personen voor de bloedstollingsstudie, ben ik met name dank verschuldigd aan Dr. Rutger Lulofs (Medische Psychologie); ook aan Drs. Cor Meesters (Medische Psychologie), Dr. Nancy Nicolson en Drs. Marleen van Eck (Socialle Psychiatrie), de vakgroep Huisartsgeneeskunde (speciaal Prof. Jan van Ree en Dr. Paul Zwietering) en het Peilstation van de GG en GD te Maastricht.

Zonder sociale steun is het vrijwel ondoenlijk om een dissertatie te schrijwen. Oprechte dank daarvoor geldt natuurlijk niet alleen mijn collegae van de vakgroepen Medische Psychologie en Geestelijke Gezondheidkunde. De paranimfen Bram Donkers en Jan van Busschbach hebben mij altijd het bellang van de grond onder de beide voeten voor ogen gehouden en me bovendien verblijd met verstrooiing in respectievelijk het maken van niet te evenaren radioprogramma's en de verdieping in allerlei bepalingen van de kwaliteit van leven (na geneeskundige interventies). De AIOvereniging Rijksuniversiteit Limburg, waarvan ik met plezier voorzitter was, leerde mij de heterogeniteit van het wetenschapsbedrijf kennen. Jolanda en Prof. Bob de Levie makkten mijn 'big move' zo eenvoudig, dat ik mijn door onderzoek en muziek geobsedeerde levensstijl zonder veel belemmering heb kunnen voortzetten in de Verenigde Staten van Amerika. Veel inspiratie en energie heb ik ontvangen van Johnny's Musicians from the Green Can (Hans, Paul en ook Dick, Bert en Inez) en mijn waarde vrienden Jan-Dirk Berkhout en Peter Onderwater. Er zijn geen toereikende woorden voor hetgeen ik mijn familie en geliefden dankbaar om ben.

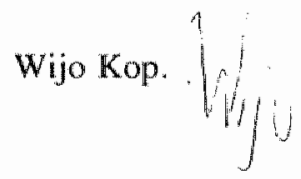




\section{Publications.}

Kop WJ, Merckelbach $H$, Muris $P$. Unilateral contraction of facial muscles and emotion; a failed replication. Cortex 1991; 27: 101-104. Schmand B, Kop WJ, Kuipers T, Bosweld J. Implicit learning in psychotic patients. Schizophrenia Res
$1992 ; 7: 55-64$.

Schagen van Leeuwen J, Koppeschaar HPF, Kop WJ, te Velde ER, Hackeng WHL. Haspels AA. Calcitonin gene-related peptide, the menstrual cycle and premenstrual syndrom. Gynecol Endocrinol $1992 ; 6: 199-204$.

Schagen van Leeuwen JH, te Velde ER, Koppeschaar HPE, Kop WJ, Thijssen JHH, van Ree JM, Haspels AA. Is premenstrual syndrome an endoctine disorder? J Psychosom Obstet Gynaecol 1993; 14: 91-109.

Kop WJ. Waarneming tijdens algahele anesthesie (boekbespreking). De Psycholoog 1993; 28: 116 117.

Appels A, Kop WJ, Meesters C, et al. Vital exhaustion and acute coronary syndromes, an update. In: Maes S (ed), International Review of Health Psychology. London: Wiley: in press.

Kop WJ, Merckelbach H, Muris P. Unilateral contraction and induction of emotion, a reply to Sehiff and Lamon. Cortex: in press.

Kop WJ, Appels A, Mendes de Leon CF, de Swart $H B_{*}$ Băr FW. The effect of successful coronary angioplasty on feelings of exhaustion. Int $\mathbb{J}$ Cardiol: in press.

Kop WJ, Appels A, Mendes de Leon CF, de Swart HB, Bär FW. Vital exhaustion predicts new cardiac events after successful coronary angioplasty. Pshchosom Med: in press.

\section{Abstracts/Proceedings.}

Kop WJ, Appels A, Mendes de Leon CF Vitale uitputting als onafhankelijke voorspeller van coronaire hartziekten. Tijdschr Soc Gezondheidszorg 1990; 69: 22.

Kop WJ, Appels A, Mendes de Leon CF. Vital exhaustion is a precursor of coronary heart disease, not explained by subclinical coronary dysfunctioning; Procedings of 31 th Dutch Federation meeting: Federation of Medical Scientific Societies 1990: p. 50

Kop WJ, Appels A, Mendes de Leon CF. Vital exhaustion is an independent precursor of coronary heart disease, not explained by subclinical coronary dysfunctioning. Proc First Int Congr Beh Med 1990 (Upsala, Sweeden): 28-30.

Kop WI, Appels A, Mendes de Leon CF. The effect of coronary angioplasty on exhaustion (paper). Psychosoma Med 1991; 53: 213.

Kop WI, Appels A, Mendes de Leon CF. Relation between exhaustion and severity of coronary artery disease. Proc 12th An Meeting Soc Beh Med 1991 (Washington DC, USA): 79. 
Kop WJ, Appels A, Mendes de Leon CF. Exhaustion after percitaneous transluminall coronary altgioplasty. Proc 12th An Meeting Soc Beh Med 1991 (Washington DC, USA): 90.

Kop WJ, Appels A, Mendes de Leon CF, Bär FW, de Swart H. Vital exhaustion predicts new cardiac events after PTCA - Final resullts (paper). Psychosom Med 1993; 55: 104-105.

Kop WJ, Hamulyak K, Appels A. The relationship between vital exhaustion and blood-cloting factors. Psychosom Med 1993; 55: 126-127.

Kop WJ, Hamulyak $K^{*}$, A.ppels A. Vital exhaustion is related to inncreased levels of blood-clotting factors. Ann Beh Med 1993; 15 sup: 118.

Kop WJ, Mendes de Leon CF, Appels A. Are feelings of exhaustion before myocardial infarction due to impaired left ventricular function? Ann Belh Med 1993; 15 sup: 118.

Kop WJ, Appels A, Mendes de Leon CF, Bär FW, de Swart HB. Vitale uitputting voorspelt het klinisch beloop na succeswolle coronaire angioplastiek. Tijdschr Soc Gezondheidszorg 1993; 71: M26. 


\section{Curriculum Vitae.}

Willem Johan Kop werd geboren op 11 augustus 1963 te Zoetermeer. Hij behaalde in 1981 het Voorbereidend Wetenschappelijk Onderwijs-B diploma aan het Christelijk Lyceum te Zeist, waarna hij begon met de studie Psychologie aan de Rijksuniversiteit Utrecht. In 1988 werd het doctoraal examen oude stijl afgelegd met als eerste hoofdrichting Functieleer en als tweede Klinische Psychologie. In deze periode was de auteur mede-oprichter van de Werkgroep Neuropsychologie Utrecht en participeerde hij in een onderzoek naar de psychologische en hormonale correlaten van het premenstrueel syndroom dat plaats vond bij de vakgroep Gynaecologie en Obstetrie van het Academisch Ziekenhuis te Utrecht (Prof. E. te Velde en Dr. J. Schagen van Leeuwen). Volgend op het afronden van de studie was hij verbonden aan de vakgroep Psychiatrie van hetzelfde ziekenhuis bij het project "Negatieve Symptomen van Psychosen" (Prof. M. Kuilman en Dr. B. Schmand), waar hij tot mei 1989 werkzaam was.

In juni 1989 volgde zijn aanstelling via de Nederlandse Organisatie voor Wetenschappelijk onderzoek bij de Rijksuniversiteit Limburg als onderzoeker in opleiding. Hij werkte bij de vakgroep Medische Psychologie bij Prof. A. Appels aan het huidige proefschrift in samenwerking met Dr. F. Bär van de vakgroep Cardiologie (hoofd, Prof. H. Wellens). Naast zijn werk als onderzoeker is hij gedurende twee jaar voorzitter geweest van de locale vereniging van assistenten in opleiding.

Momenteel is de auteur als research associate verbonden aan de vakgroep Medical and Clinical Psychology van de Uniformed Services University of the Health Sciences te Bethesda, Maryland, Verenigde Staten van Amerika, waar hij bij Prof. D. Krantz onderzoek doet naar de invloed van lichamelijke en psychische belasting op het ontstaan van myocardiale ischemie. 